Journal of Applied AnALysis

Vol. 8, No. 2 (2002), pp. 153-200

\title{
MATHEMATICAL ANALYSIS AND OPTIMAL CONTROL PROBLEMS FOR THE PERTURBATION OF THE PRIMITIVE EQUATIONS OF THE OCEAN WITH VERTICAL VISCOSITY
}

\author{
A. BELMILOUDI
}

Received February 28, 2001

\begin{abstract}
In this paper we consider an oceanic domain in $\mathbb{R}^{3}$, in which there exists, at initial time, a current $U_{0}$, a pressure $p_{0}$ and a density $\rho_{0}$. The perturbation $U, p$ and $\rho$ of the velocity, the pressure and the density are induced by a perturbation of the mean windstress. The equations are of Navier-Stokes type for the velocity and pressure, of transport-diffusion type for the density. They are linearized around a given mean circulation and modified by the physical assumptions including the Boussinesq approximation and the Hydrostatic approximation with vertical viscosity. The existence and uniqueness of the solution for the variational problem are studied for the three-dimensional problem, and for the two-dimensional cyclic problem derived by assuming a sinusoidal $x$-dependence for the perturbation of mean flow. The latter corresponds to a modelization of tropical instability waves which are illustrated by El Nino phenomenon.
\end{abstract}

2000 Mathematics Subject Classification. 35Q30, 37N10, 49J20, 49K20, 65J10, 76D05, $76 \mathrm{U} 05,86 \mathrm{~A} 22$.

Key words and phrases. Navier-Stokes type, primitive equations, regularity in domains with corners, optimal control, assimilation of surface data, equatorial waves, oceanography.

ISSN 1425-6908 C Heldermann Verlag. 


\begin{abstract}
. We want to choose the value of the surface pressure $P$ as the observation of the method of control. To define that quantity, we have proved the $H^{2}$-regularity of the perturbation $(\mathbf{u}, \rho)$ and then the $L^{2}$-regularity of the surface pressure $P$. This result is valid in a domain with corners. It is proved by means of an extension method, with even-odd reflection, provided the perturbation of the wind-stress is sufficiently regular and satisfies compatibility relations. We then develop a method of control in order to calculate the current corresponding to the observed sea level in a fluid domain and during a time $\mathrm{T}$. The variability of the surface pressure $P$, deduced from altimetric measurements, constitues the observation. The control is the variability of wind-stress $\mathbf{f}$, which acts as to forcing of the perturbation. The cost function measures the distance between the observed and computed surface pressure. We prove the existence and uniqueness of the optimal control, which is characterized by a set of equations including the direct problem and the adjoint problem. These results are valid for the three-dimensional problem and the two-dimensional cyclic problem.
\end{abstract}

\title{
1. Introduction
}

The oceanic phenomenon that we want to modelize occurs in the tropical Atlantic and Pacific oceans. The oceanic circulation there is characterized by steady zonal currents and by long waves propagating westward along the equator, driven by the variability of the wind-stress and superimposed to the mean currents. The equatorial waves can be connected with strong vertical velocities and then induce "upwellings" or "downwellings". These phenomena modify the properties of the sea water near the surface: temperature, plankton etc., and are therefore of great importance for climate, fishing activities etc. These equatorial waves have been evidenced from in situ observations, and more recently from altimetric measurements [3], [5], [6], [7], [8], [10]. Numerical simulations have also carried out in order to analyze the process of wave generation. Such numerical models are described in [3], [4], [11], [12].

In this paper, we are going to study the mathematical properties of the problem related to the modelization of equatorial waves. The whole current can be considered as the sum of two terms: the mean current which is known for each tropical season, and a perturbation term corresponding to the waves.

We consider an oceanic domain $\Omega$ extending on both sides of the equator $\left(10^{0} S-10^{0} \mathrm{~N}\right)$, and of constant depth $H$. The curvature of the earth is neglected. The vertical extension of $\Omega(-H \leq z \leq 0)$ corresponds to a part of the physical domain. We assume that for depths greater than $H$, the variability is negligible. The perturbation of the current is made of 
zonal propagating waves. So, we can choose the zonal extension of $\Omega$ as the greatest wavelength, and impose periodic conditions on the eastern and western boundaries.

The equations of motion in an oceanic domain are derived from NavierStokes system but take into account oceanographical assumptions such as Hydrostatic approximation with vertical viscosity, i.e. (1.2) and Boussinesq approximation, i.e. the density variations are neglected except in the terms of gravity acceleration. Equations and boundary conditions are written in order to fit with physical features and then differ from the classical NavierStokes case.

The equations satisfied by total velocity $U=(\mathbf{u}, w)=(u, v, w)$, density $\rho$ and pressure $p$ are:

$$
\begin{aligned}
& \frac{\partial U}{\partial t}+\left(U \cdot \nabla_{3}\right) U+\mathbf{F} \wedge U-\operatorname{div}_{3}\left(\nu_{1} \nabla_{3} U\right)+\frac{1}{\rho} \nabla_{3} p=\mathbf{G}, \\
& \frac{\partial \rho}{\partial t}+\left(U \cdot \nabla_{3}\right) \rho-\operatorname{div}_{3}\left(\nu_{2} \nabla_{3} \rho\right)=0 \\
& \operatorname{div}_{3}(U)=0
\end{aligned}
$$

$(x, y, z)$ are the cartesian coordinates: $x, y$ are measured in the horizontal plane of the undisturbed sea-surface ( $x$ towards the east, $y$ towards the north) and $z$ is vertically ascendant. $\mathbf{F}=(0,0,2 \omega \sin \phi)$ is the Coriolis force, $\omega$ the rotation rate of earth, $\phi$ the latitude. $\mathbf{G}=(0,0,-g)$ is the gravity force. $\nabla_{3}=\left(\frac{\partial}{\partial x}, \frac{\partial}{\partial y}, \frac{\partial}{\partial z}\right), \operatorname{div}_{3} U=\nabla_{3} \cdot U$.

Turbulence is modelized by the dissipative terms. Observation of turbulent flows leads distinguish between horizontal and vertical mixing: $\nu_{1 h}$ and $\nu_{2 h}$ (resp. $\nu_{1 v}$ and $\nu_{2 v}$ ) denote the coefficients of horizontal (resp. vertical) eddy viscosity and diffusivity. According to the parametrization described in [23], [24] $\nu_{1 v}$ and $\nu_{2 v}$ are variable, positive and bounded functions. They are deduced from the given mean circulation. $\nu_{1 h}$ and $\nu_{2 h}$ are constant and positive.

We are now going to simplify equations (1.1). It is known that the differences of the density of the sea water are significant only in the term of buoyancy and in the equation of state. Density $\rho$ appearing in the right hand term has to satisfy the second part of equation (1.1). On the other hand, density will be assumed to be constant and equal to a mean value $\rho_{\text {moy }}$ in the equations describing the horizontal motion. This assumption is known as Boussinesq approximation. The horizontal scale is much bigger than the vertical one. Moreover the viscosity will play an important role in the dynamics of the ocean. Therefore, we can replace the equation describing the vertical motion by the following equations called by the Hydrostatic 
approximation with vertical viscosity [20]:

$$
-\nu_{1 h} \Delta w-\frac{\partial}{\partial z}\left(\nu_{1 v} \frac{\partial w}{\partial z}\right)+\frac{1}{\rho_{\text {moy }}} \frac{\partial p}{\partial z}+\frac{\rho}{\rho_{\text {moy }}} g=0 .
$$

Applying these physical assumptions to (1.1), we obtain the following equations:

$$
\begin{aligned}
& \frac{\partial \mathbf{u}}{\partial t}+(\mathbf{u} . \nabla) \mathbf{u}+w \frac{\partial \mathbf{u}}{\partial z}+\mathbf{F} \wedge_{2} \mathbf{u}-\nu_{1 h} \Delta \mathbf{u}-\frac{\partial}{\partial z}\left(\nu_{1 v} \frac{\partial \mathbf{u}}{\partial z}\right)+\frac{1}{\rho_{m o y}} \nabla p=0 \\
& -\nu_{1 h} \Delta w-\frac{\partial}{\partial z}\left(\nu_{1 v} \frac{\partial w}{\partial z}\right)+\frac{1}{\rho_{m o y}} \frac{\partial p}{\partial z}+\frac{\rho}{\rho_{m o y}} g=0 \\
& \frac{\partial \rho}{\partial t}+(\mathbf{u} . \nabla) \rho+w \frac{\partial \rho}{\partial z}-\nu_{2 h} \Delta \rho-\frac{\partial}{\partial z}\left(\nu_{2 v} \frac{\partial \rho}{\partial z}\right)=0 \\
& \operatorname{div}(\mathbf{u})+\frac{\partial w}{\partial z}=0
\end{aligned}
$$

with $\mathbf{F} \wedge_{2} \mathbf{u}=\mathbf{F} \wedge(\mathbf{u}, 0)$.

We now split up the circulation $(\mathbf{u}, w, \rho, p)$ into a given mean value $\left(\mathbf{u}_{0}, w_{0}, \rho_{0}, p_{0}\right)$ and a variability $\left(\mathbf{u}^{\prime}, w^{\prime}, \rho^{\prime}, p^{\prime}\right)$ that will be computed by the model. This expansion is justified in tropical oceans: the steady mean circulation $\left(\mathbf{u}_{\mathbf{0}}, w_{0}, \rho_{0}, p_{0}\right)$ is known for each tropical season; the variability $\left(\mathbf{u}^{\prime}, w^{\prime}, \rho^{\prime}, p^{\prime}\right)$ is made of westward propagating waves. $\left(\mathbf{u}_{\mathbf{0}}, w_{0}, \rho_{0}, p_{0}\right)$ has to satisfy steady state equations (1.3).

The equations satisfied by the variability $\left(\mathbf{u}^{\prime}, w^{\prime}, \rho^{\prime}, p^{\prime}\right)$ can be deduced from (1.3). They can be written:

$$
\begin{aligned}
& \frac{\partial \mathbf{u}^{\prime}}{\partial t}+\left(\mathbf{u}^{\prime} \cdot \nabla\right) \mathbf{u}^{\prime}+w^{\prime} \frac{\partial \mathbf{u}^{\prime}}{\partial z}+\left(\mathbf{u}_{\mathbf{0}} \cdot \nabla\right) \mathbf{u}^{\prime}+w_{0} \frac{\partial \mathbf{u}^{\prime}}{\partial z}+\left(\mathbf{u}^{\prime} . \nabla\right) \mathbf{u}_{\mathbf{0}}+w^{\prime} \frac{\partial \mathbf{u}_{\mathbf{0}}}{\partial z} \\
& +\mathbf{F} \wedge_{2} \mathbf{u}^{\prime}-\nu_{1 h} \Delta \mathbf{u}^{\prime}-\frac{\partial}{\partial z}\left(\nu_{1 v} \frac{\partial \mathbf{u}^{\prime}}{\partial z}\right)+\frac{1}{\rho_{\text {moy }}} \nabla p^{\prime}=0 \\
& -\nu_{1 h} \Delta w^{\prime}-\frac{\partial}{\partial z}\left(\nu_{1 v} \frac{\partial w^{\prime}}{\partial z}\right)+\frac{1}{\rho_{m o y}} \frac{\partial p^{\prime}}{\partial z}+\frac{\rho^{\prime}}{\rho_{m o y}} g=0 \\
& \frac{\partial \rho^{\prime}}{\partial t}+\left(\mathbf{u}^{\prime} \cdot \nabla\right) \rho^{\prime}+w^{\prime} \frac{\partial \rho^{\prime}}{\partial z}+\left(\mathbf{u}^{\prime} \cdot \nabla\right) \rho_{0}+w^{\prime} \frac{\partial \rho_{0}}{\partial z}+\left(\mathbf{u}_{\mathbf{0}} \cdot \nabla\right) \rho^{\prime} \\
& \quad+w_{0} \frac{\partial \rho^{\prime}}{\partial z}-\nu_{2 h} \Delta \rho^{\prime}-\frac{\partial}{\partial z}\left(\nu_{2 v} \frac{\partial \rho^{\prime}}{\partial z}\right)=0 \\
& \operatorname{div}\left(\mathbf{u}^{\prime}\right)+\frac{\partial w^{\prime}}{\partial z}=0
\end{aligned}
$$

Nota bene: notation " $\prime$ " used for the perturbation of the mean flow will now be omitted.

The oceanic domain $\Omega$ can be defined as $\Omega=] 0, L x[\times]-L y, L y[\times]-H, 0[$. $\Gamma$ denotes its boundary: $\Gamma=\Gamma_{0} \cup \Gamma_{5} \cup \Gamma_{1} \cup \Gamma_{2} \cup \Gamma_{3} \cup \Gamma_{4}$ where $\Gamma_{0}$ denotes 
the surface $(z=0), \Gamma_{5}$ the bottom $(z=-H), \Gamma_{1}$ and $\Gamma_{2}$ the eastern and western boundaries, $\Gamma_{3}$ and $\Gamma_{4}$ the northern and southern boundaries.

We note that the vertical extension $-H \leq z \leq 0$ of the domain $\Omega$ does not correspond to the physical ocean but only to the layer where the variability is computed. We assume that for depths greater than $H$ the perturbation of the mean flow is negligible. The perturbation is not computed in the thin surface layer $0 \leq z \leq \xi$, where $z=\xi$ is the free sea surface. To take into account the phenomena we want to describe we set mixed boundary conditions:

- the flow is periodic in the $x$-direction:

$\left.\mathbf{u}\right|_{\Gamma_{1}}=\left.\mathbf{u}\right|_{\Gamma_{2}},\left.w\right|_{\Gamma_{1}}=\left.w\right|_{\Gamma_{2}}$ and $\left.\rho\right|_{\Gamma_{1}}=\left.\rho\right|_{\Gamma_{2}}$,

- on the northern and southern boundaries we impose a sliding condition for the flow and an homogeneous Dirichlet condition for the perturbation of the density:

$v=\rho=0, \frac{\partial u}{\partial y}=0$ and $\frac{\partial w}{\partial y}=0$ on $\Gamma_{3}$ and $\Gamma_{4}$,

- the perturbation vanishes at $z=-H$ :

$\mathbf{u}=\mathbf{0}, w=0$ and $\rho=0$ on $\Gamma_{5}$,

- the perturbation is driven by the perturbation of the wind-stress $\mathbf{f}$

$$
w=0, \frac{\partial u}{\partial z}=\frac{f_{1}}{\nu_{1 v}}, \frac{\partial v}{\partial z}=\frac{f_{2}}{\nu_{1 v}} \text { and } \frac{\partial \rho}{\partial z}=0 \text { on } \Gamma_{0},
$$

with $\mathbf{f}=\left(f_{1}, f_{2}\right), \mathbf{u}=(u, v)$.

We assume that, at initial time $t=0$, the mean circulation is not disturbed. Therefore the initial condition is:

$$
\mathbf{u}(t=0)=\mathbf{0}, \rho(t=0)=0 .
$$

The equations (1.4) are not of a Cauchy-Kovalevsky type with respect to all variables because of the continuity equation and of the Hydrostatic equation with vertical viscosity (1.2). The method proposed by Lions-Temam-Wang in [19], [20] is the integration of these two diagnostic equations with respect to the vertical variable, and then we obtain another formulation of the primitive equations, which is an three dimensional evolution system. Therefore we can used Faedo-Galerkin method to solve the weak formulations. By integrating the continuity equation with respect to $z$ and taking the boundary conditions for $w$ into account, we obtain

$$
\begin{aligned}
& w(x, y, z ; t)=W(\mathbf{u})(x, y, z ; t)=-\operatorname{div}\left(\int_{-H}^{z} \mathbf{u}\left(z^{\prime}\right) d z^{\prime}\right), \\
& -\operatorname{div}\left(\int_{-H}^{0} \mathbf{u}\left(z^{\prime}\right) d z^{\prime}\right)=0
\end{aligned}
$$


By integrating the Hydrostatic equation with vertical viscosity in (1.4), we obtain:

$$
\frac{p}{\rho_{\text {moy }}}=\frac{P}{\rho_{\text {moy }}}+\frac{g}{\rho_{\text {moy }}} \int_{z}^{0} \rho\left(z^{\prime}\right) d z^{\prime}+\int_{z}^{0} L(\mathbf{u})\left(z^{\prime}\right) d z^{\prime}
$$

where

$$
L(\mathbf{u})=-\nu_{1 h} \Delta W(\mathbf{u})-\frac{\partial}{\partial z}\left(\nu_{1 v} \frac{\partial W(\mathbf{u})}{\partial z}\right)
$$

and $P$ is the pressure of the sea water on the surface of the ocean. Then by using (1.7), (1.8) and after linearization around the steady mean solution we obtain the following reformulation of equations (1.4):

$$
\begin{aligned}
& \frac{\partial \mathbf{u}}{\partial t}+\left(\mathbf{u}_{\mathbf{0}} \cdot \nabla\right) \mathbf{u}+w_{0} \frac{\partial \mathbf{u}}{\partial z}+(\mathbf{u} . \nabla) \mathbf{u}_{\mathbf{0}}+W(\mathbf{u}) \frac{\partial \mathbf{u}_{\mathbf{0}}}{\partial z}+\mathbf{F} \wedge_{2} \mathbf{u} \\
& \quad-\nu_{1 h} \Delta \mathbf{u}-\frac{\partial}{\partial z}\left(\nu_{1 v} \frac{\partial \mathbf{u}}{\partial z}\right)+\frac{1}{\rho_{\text {moy }}} \nabla P+\frac{g}{\rho_{\text {moy }}} \nabla R(\rho)+\nabla \mathcal{L}(\mathbf{u})=0, \\
& \frac{\partial \rho}{\partial t}+(\mathbf{u} . \nabla) \rho_{0}+W(\mathbf{u}) \frac{\partial \rho_{0}}{\partial z}+\left(\mathbf{u}_{\mathbf{0}} \cdot \nabla\right) \rho+w_{0} \frac{\partial \rho}{\partial z} \\
& \quad-\nu_{2 h} \Delta \rho-\frac{\partial}{\partial z}\left(\nu_{2 v} \frac{\partial \rho}{\partial z}\right)=0, \\
& \operatorname{div}\left(\int_{-H}^{0} \mathbf{u}\left(z^{\prime}\right) d z^{\prime}\right)=0
\end{aligned}
$$

where $R(\rho)=\int_{z}^{0} \rho\left(z^{\prime}\right) d z^{\prime}$ and $\mathcal{L}(\mathbf{u})=\int_{z}^{0} L(\mathbf{u})\left(z^{\prime}\right) d z^{\prime}$.

Remark 1.1. The above system is three dimensional, but the unknown function $P$ is only a function of $x, y$ and time $t$.

The problem obtained is studied and we prove the existence and the uniqueness of the perturbation of the mean circulation. The surface-pressure associated with that solution is not sufficiently regular to use that as the observation of the method of the control. We therefore prove a regularity result for the perturbation. This result is valid in a domain $\Omega$ with corners. The presence of corners, prevents us from applying a standard theorem of regularity. Next we develop a control method in order to compute the variability of the circulation in an oceanic domain from satellite measurements which are obviously surface observations. Altimetric measurements give the distance between the satellite and the sea surface. It is now possible to extract from these data the sea level variability with a precision in order of centimeters. The observation for the control method is the variability of the pressure, which is the level of the undisturbed sea surface. This data can be deduce from the variability of free sea surface, given by altimetric measurements (the free sea surface is unknown, only its perturbation is given by altimetry). In order to obtain the relationship between pressure and 
free sea surface, we have to integrate the equation of hydrostatic pressure, with respect to $z$, between the undisturbed sea surface and to neglect the variability of the atmospheric pressure at the sea surface.

The perturbation being made of zonally propagating waves, we construct a cyclic model by assuming the following $x$-dependence: for each perturbated real-function (vector or scalar) $C$, we denote by $\tilde{C}$ the complexfunction independant of $x$ such that:

$$
C(x, y, z ; t)=e^{i m x} \tilde{C}(y, z ; t) .
$$

The wavelength $\lambda=2 \pi / m$ will be fixed for each numerical run. We thus obtain a process model focusing on "instability waves" which are characterized by eddies of a time-space scale of 30 days-1000 kms propagating westward along the equator. The model doesn't include the reflection on coasts which gives rise to eastward propagating waves of less importance in the energetic balance of tropical oceans. According to physical features, we neglect the $x$-dependence of $\left(\mathbf{u}_{\mathbf{0}}, w_{0}, \rho_{0}\right)$ : the mean circulation is given so that $\mathbf{u}_{\mathbf{0}}=\mathbf{u}_{\mathbf{0}}(y, z), w_{0}=w_{0}(y, z), \rho_{0}=\rho_{0}(y, z)$. Since $\nu_{1 v}$ and $\nu_{2 v}$ are depending on the given mean $\left(\mathbf{u}_{\mathbf{0}}, w_{0}, \rho_{0}\right), \nu_{1 v}$ and $\nu_{2 v}$ are then supposed to vary with respect to $y$ and $z$. The periodic method reduces the initial three-dimensional problem to a two-dimensional one.

According to these assumptions, the equations satisfied by the perturbation are the following (for each fixed value of the wave number $\mathrm{m}$ ):

$$
\begin{aligned}
& \frac{\partial \tilde{\mathbf{u}}}{\partial t}+\left(\mathbf{u}_{\mathbf{0}} \cdot \nabla_{m}\right) \tilde{\mathbf{u}}+w_{0} \frac{\partial \tilde{\mathbf{u}}}{\partial z}+\left(\tilde{\mathbf{u}} \cdot \nabla_{0}\right) \mathbf{u}_{\mathbf{0}}+W_{m}(\tilde{\mathbf{u}}) \frac{\partial \mathbf{u}_{\mathbf{0}}}{\partial z}+\mathbf{F} \wedge_{2} \tilde{\mathbf{u}}-\nu_{1 h} \Delta_{m} \tilde{\mathbf{u}} \\
& \quad-\frac{\partial}{\partial z}\left(\nu_{1 v} \frac{\partial \tilde{\mathbf{u}}}{\partial z}\right)+\frac{1}{\rho_{m o y}} \nabla_{m} \tilde{P}+\frac{g}{\rho_{m o y}} \nabla_{m} R(\tilde{\rho})+\nabla_{m} \mathcal{L}_{m}(\tilde{\mathbf{u}})=0 \\
& \frac{\partial \tilde{\rho}}{\partial t}+\left(\tilde{\mathbf{u}} . \nabla_{0}\right) \rho_{0}+W_{m}(\tilde{\mathbf{u}}) \frac{\partial \rho_{0}}{\partial z}+\left(\mathbf{u}_{\mathbf{0}} \cdot \nabla_{m}\right) \tilde{\rho}+w_{0} \frac{\partial \tilde{\rho}}{\partial z}-\nu_{2 h} \Delta_{m} \tilde{\rho} \\
& \quad-\frac{\partial}{\partial z}\left(\nu_{2 v} \frac{\partial \tilde{\rho}}{\partial z}\right)=0 \\
& \operatorname{div}_{m}\left(\int_{-H}^{0} \tilde{\mathbf{u}}\left(z^{\prime}\right) d z^{\prime}\right)=0
\end{aligned}
$$

where

$$
\begin{aligned}
& W_{m}(\tilde{\mathbf{u}})=-\int_{-H}^{z} \operatorname{div}_{m}(\tilde{\mathbf{u}}) d z^{\prime}, \\
& \mathcal{L}_{m}(\tilde{\mathbf{u}})=\int_{z}^{0} L_{m}(\tilde{\mathbf{u}})\left(z^{\prime}\right) d z^{\prime}, \\
& L_{m}(\tilde{\mathbf{u}})=-\nu_{1 h} \Delta_{m} W_{m}(\tilde{\mathbf{u}})-\frac{\partial}{\partial z}\left(\nu_{1 v} \frac{\partial W_{m}(\tilde{\mathbf{u}})}{\partial z}\right),
\end{aligned}
$$




$$
\begin{aligned}
& \nabla_{m}=\left(i m, \frac{\partial}{\partial y}\right), \operatorname{div}_{m} \tilde{\mathbf{u}}=\nabla_{m} \cdot \tilde{\mathbf{u}}=i m \tilde{u}+\frac{\partial \tilde{v}}{\partial y}, \Delta_{m}=-m^{2}+\frac{\partial^{2}}{\partial y^{2}} \\
& \nabla_{0}=\left(0, \frac{\partial}{\partial y}\right) .
\end{aligned}
$$

Nota bene: notation " " used for the perturbation of the mean flow will now be omitted. The cyclic model uses complex variables: $\mathbf{u} \in \mathbb{C}^{2}$, $\rho \in \mathbb{C}, P \in \mathbb{C}$. The physically significant perturbation corresponding to each complex function is given by its real part.

Problem (1.10) is posed in $\omega \times] 0, T[$ with $\omega=]-L y, L y[\times]-H, 0\left[\subset \mathbb{R}^{2}\right.$.

Set $\gamma=\gamma_{0} \cup \gamma_{5} \cup \gamma_{3} \cup \gamma_{4}$ the boundary of $\omega, \gamma_{0}$ denotes the surface $(z=0)$, $\gamma_{5}$ the bottom $(z=-H), \gamma_{3}$ and $\gamma_{4}$ the northern and southern boundaries. The boundary conditions deduced from (1.5) are the following:

- $v=\rho=0, \frac{\partial u}{\partial y}=0$ and $\frac{\partial W_{m}(\mathbf{u})}{\partial y}=0$ on $\gamma_{3}$ and $\gamma_{4}$,

- $\mathbf{u}=\mathbf{0}, W_{m}(\mathbf{u})=0$ and $\rho=0$ on $\gamma_{5}$,

- $W_{m}(\mathbf{u})=0, \frac{\partial u}{\partial z}=\frac{f_{1}}{\nu_{1 v}}, \frac{\partial v}{\partial z}=\frac{f_{2}}{\nu_{1 v}}$ and $\frac{\partial \rho}{\partial z}=0$ on $\gamma_{0}$,

The variability of the pressure on $\Gamma_{0}$ (resp. $\gamma_{0}$ ), deduced from altimetric data, constitues the observation. The variability of the windstress $\mathbf{f}$ acts as the forcing of the perturbation and is unknown. We take $\mathbf{f}$ as the control, $(\mathbf{u}(\mathbf{f}), \rho(\mathbf{f}), P(\mathbf{f}))$ are the velocity, the density and the surface pressure corresponding to any control $\mathbf{f}$ and satisfying problem (1.9), (1.5), (1.6) (resp. (1.10), (1.11), (1.6)). The optimal control is defined as the windstress minimizing a given cost function which measures the distance between the observed pressure and the surface pressure $P(\mathbf{f})$. The pressure observed on $\Gamma_{0}$ (resp. $\left.\gamma_{0}\right)$ is the trace of a circulation $\left(\mathbf{u}_{r c}, \rho_{r c}, p_{r c}\right)$ driven by a wind $\mathbf{f}_{r c}$. The solution $(\mathbf{u}(\mathbf{f}), \rho(\mathbf{f}), P(\mathbf{f}))$ induced by the optimal control $\mathbf{f}$ has to approach the real circulation. The control method then makes it possible to compute the circulation in all the domain $\Omega$ by means of data on a part of the boundary.

In order to solve the problem of optimal control, we have to consider the adjoint equations associated to (1.9), (1.5), (1.6) (resp. (1.10), (1.11), (1.6)). We then apply the control theory introduced by Lions [17]. More recently, G. I. Marchuk developed the method of adjoint equations in mathematical physics and performed variational data assimilation in environmental problems [21], [22]. The classical control problem arising in meteorology or oceanography is the adjustment of the initial condition in order to velocity and temperature fields which agree with observation in situ obtain. Our purpose is quite different since we control by the variability of the windstress in order to restitute the observed surface pressure deduced from Geosat altimetric measurements. So, the control acts as Neumann condition on a part 
of the boundary, the surface pressure is the observed quantity and requires the use a mixed velocity-pressure formulation. Controls and observations are thus defined on a part of the boundary. Moreover the nature of the equations and especially the non-local contraint the third part of (1.9) add difficulties.

This paper is organized as follows:

Section 2 is devoted to the study of the three dimensional problem (1.9), (1.5), (1.6), linearized around the mean circulation. We prove the existence and uniqueness of a perturbation $(\mathbf{u}, \rho, P)$. According to the hydrostatic approximation with vertical viscosity, this problem is not of classical NavierStokes type. The horizontal velocity is given by a pronostic equation while the vertical velocity verifies a diagnostatic equation. This type of problem has been studied by Lions, Temam and Wang [19], [20] but in this paper the formulation is quite different by advection terms related to the mean circulation, and by specific boundary conditions. We prove a regularity result for perturbation $(\mathbf{u}, \rho, P)$. The shape of the oceanic domain $\Omega$, and especially the presence of corners, prevents us from applying a standard theorem of regularity. We assume some regularity, in space and time, on the perturbation of the wind-stress and on the mean flow, and we impose the compatibility conditions. The regularity result is obtained by means of an extension method by even-odd reflection introduced in [9]. The regularity result of this type of problem, in stationnary case with constant viscosity and diffusivity and a "cylindric-type" domain, has been studied by Ziane in [26] by using the results obtained by Dauge in [14], [15] and Grisvard in [16]. Our purpose is different. Firstly we study the perturbation of time-dependant primitive equations in a "cubic-type" domain around the mean flow $\left(U_{0}, \rho_{0}, p_{0}\right)$. The turbulence is modelized by the dissipative terms. Observation of turbulent flows leads distinguish between horizontal and vertical mixing. According to the parametrization described in [24], the vertical viscosity and diffusivity are variables, positives and bounded functions. Consequently different operators and then difficulties are appearing. Secondly, we impose other boundary conditions (1.5). These mixed boundary conditions are not too restrictive: they are applied not to the total circulation, but only to its perturbation. Moreover the nature of these conditions permits to use the extension method by even-odd reflexion to obtain the regularity results. Next we study the problem of control. We prove the existence and uniqueness of the solution. The optimal control is given as a function of $\left(\mathbf{u}^{*}, \rho^{*}, P^{*}\right)$, the solution of the adjoint problem associated with the direct problem (1.9), (1.5), (1.6). We thus obtain a set of equations characterizing the optimal control.

Section 3 is devoted to the study of the two dimensional problem (1.10), (1.11), (1.6). The plan is the same as in Section 2: we study the properties 
of operators appearing in the weak formulation and then prove the existence and uniqueness of a solution. As in Section 2 we give sufficient conditions on the perturbation of the windstress $\mathbf{f}$ for to prove the regularity result. Finally, we study the optimal control problem. In this section operators are modified by the cyclic asumption: they depend on the wavenumber $\mathrm{m}$ and the functions are complex valued. The main difference between the present problem and problem studied in [6] is that the equations in [6] are of Cauchy-Kovalevsky type with respect to all variables.

\section{The three dimensional problem}

\subsection{Variational formulation.}

In order to study problem (1.9), we introduce the following functional spaces:

$$
\begin{aligned}
& \mathcal{V}_{0}=\left\{(\mathbf{v}, w) \in\left(H^{1}(\Omega)\right)^{3} \mid \operatorname{div}(\mathbf{v})+\frac{\partial w}{\partial z}=0,(\mathbf{v}, w) \cdot \mathbf{n}=0 \text { on } \Gamma\right\}, \\
& \mathcal{H}_{1}=\left\{\mathbf{v} \in\left(L^{2}(\Omega)\right)^{2},(\mathbf{v}, 0) \cdot \mathbf{n}=0\right. \\
&\text { on } \left.\Gamma_{0} \cup \Gamma_{3} \cup \Gamma_{4} \cup \Gamma_{5},\left.(\mathbf{v}, 0) \cdot \mathbf{n}\right|_{\Gamma_{1}}=-\left.(\mathbf{v}, 0) \cdot \mathbf{n}\right|_{\Gamma_{2}}\right\}, \\
& \mathcal{W}_{1}=\left\{\mathbf{v} \in\left(H^{1}(\Omega)\right)^{2} \mid W(\mathbf{v})=-\int_{-H}^{z} \operatorname{div}(\mathbf{v}) d z^{\prime} \in H^{1}(\Omega),\right. \\
&\left.\quad \mathbf{v}=\mathbf{0} \text { on } \Gamma_{5},(\mathbf{v}, 0) \cdot \mathbf{n}=0 \text { on } \Gamma_{0} \cup \Gamma_{3} \cup \Gamma_{4},\left.\mathbf{v}\right|_{\Gamma_{1}}=\left.\mathbf{v}\right|_{\Gamma_{2}}\right\}, \\
& \mathcal{V}_{1}=\left\{\mathbf{v} \in \mathcal{W}_{1} \mid \int_{-H}^{0} \operatorname{div}(\mathbf{v}) d z=0\right\}, \\
& \mathcal{H}_{2}= L^{2}(\Omega), \\
& \mathcal{V}_{2}=\left\{\phi \in H^{1}(\Omega) \mid \phi=0 \text { on } \Gamma_{3} \cup \Gamma_{4} \cup \Gamma_{5},\left.\phi\right|_{\Gamma_{1}}=\left.\phi\right|_{\Gamma_{2}}\right\},
\end{aligned}
$$

where, $\mathbf{n}$ is the unit outward vector normal to $\Gamma$.

\section{Remark 2.1.}

1) The semi-norm $|\cdot|_{1, \Omega}$ and the norm $\|\cdot\|_{1, \Omega}$ defined on $H^{1}(\Omega)$ are equivalent in $\mathcal{W}_{1}$ (resp. $\mathcal{V}_{1}$ ) and $\mathcal{V}_{2}$. We set $\|\mathbf{v}\|=|\mathbf{v}|_{1, \Omega},\|\phi\|=$ $|\phi|_{1, \Omega}$ where $|\mathbf{v}|$ and $|\phi|$ denotes the norm in $L^{2}(\Omega) ;|\mathbf{f}|_{\Gamma_{0}}$ and $(\mathbf{f}, \mathbf{v})_{\Gamma_{0}}$ denote the norm and the scalar product in $L^{2}\left(\Gamma_{0}\right)$.

2) $\mathcal{W}_{1}$ (resp. $\mathcal{V}_{1}$ ) is equipped with the following norm:

$$
\|\mathbf{u}\|_{W}^{2}=\|\mathbf{u}\|^{2}+\left\|\int_{-H}^{z} \operatorname{div}(\mathbf{u}) d z^{\prime}\right\|^{2}, \forall \mathbf{u} \in \mathcal{W}_{1}\left(\operatorname{resp} . \in \mathcal{V}_{1}\right)
$$

and $\mathcal{V}_{2}$ is equipped with the norm $\|\phi\|, \forall \phi \in \mathcal{V}_{2}$ 


\section{Lemma 2.1.}

$$
\begin{aligned}
& (\nabla \mathcal{L}(\mathbf{u}), \mathbf{v})=\nu_{1 h}\left(\nabla\left(\int_{-H}^{z} \operatorname{div}(\mathbf{u}) d z^{\prime}\right), \nabla\left(\int_{-H}^{z} \operatorname{div}(\mathbf{v}) d z^{\prime}\right)\right) \\
& \quad+\left(\nu_{1 v} \operatorname{div}(\mathbf{u}), \operatorname{div}(\mathbf{v})\right)-\left(\nu_{1 v} \gamma_{0}(\operatorname{div}(\mathbf{u})), \int_{-H}^{0} \operatorname{div}(\mathbf{v}) d z\right) \\
& \forall(\mathbf{u}, \mathbf{v}) \in\left(\mathcal{W}_{1} \cap H^{2}(\Omega)\right)^{2}
\end{aligned}
$$

Proof.

$$
(\nabla \mathcal{L}(\mathbf{u}), \mathbf{v})=\int_{\Omega} \nabla\left(\int_{z}^{0} L(\mathbf{u}) d z^{\prime}\right) \cdot \mathbf{v} d \Omega
$$

By using Green's formula we obtain

$$
(\nabla \mathcal{L}(\mathbf{u}), \mathbf{v})=-\int_{\Omega}\left(\int_{z}^{0} L(\mathbf{u}) d z^{\prime}\right) \operatorname{div}(\mathbf{v}) d \Omega+\int_{\Gamma}\left(\int_{z}^{0} L(\mathbf{u}) d z^{\prime}\right)(\mathbf{v}, 0) . \mathbf{n} d \Gamma
$$

As $\mathbf{v}$ is any element in $\mathcal{W}_{1}$, we obtain:

$$
(\nabla \mathcal{L}(\mathbf{u}), \mathbf{v})=-\int_{\Gamma_{0}}\left(\int_{-H}^{0}\left(\operatorname{div}(\mathbf{v}) \int_{z}^{0} L(\mathbf{u}) d z^{\prime}\right) d z\right) d \Gamma_{0} .
$$

By integrating by part with respect to $z$, we have

$$
(\nabla \mathcal{L}(\mathbf{u}), \mathbf{v})=\int_{\Omega}\left(L(\mathbf{u}) \int_{-H}^{z} \operatorname{div}(\mathbf{v}) d z^{\prime}\right) d \Omega .
$$

Since

$$
L(\mathbf{u})=-\nu_{1 h} \Delta W(\mathbf{u})-\frac{\partial}{\partial z}\left(\nu_{1 v} \frac{\partial W(\mathbf{u})}{\partial z}\right),
$$

by applying Green's formula, we obtain

$$
\begin{aligned}
(\nabla \mathcal{L}(\mathbf{u}), \mathbf{v})= & \nu_{1 h}\left(\nabla\left(\int_{-H}^{z} \operatorname{div}(\mathbf{u}) d z^{\prime}\right), \nabla\left(\int_{-H}^{z} \operatorname{div}(\mathbf{v}) d z^{\prime}\right)\right) \\
& +\left(\nu_{1 v} \operatorname{div}(\mathbf{u}), \operatorname{div}(\mathbf{v})\right)-\left(\nu_{1 v} \gamma_{0}(\operatorname{div}(\mathbf{u})), \int_{-H}^{0} \operatorname{div}(\mathbf{v}) d z^{\prime}\right)_{\Gamma_{0}}
\end{aligned}
$$

Remark 2.2. If $\mathbf{v} \in \mathcal{V}_{1}$ we have

$$
\begin{aligned}
(\nabla \mathcal{L}(\mathbf{u}), \mathbf{v})= & \nu_{1 h}\left(\nabla\left(\int_{-H}^{z} \operatorname{div}(\mathbf{u}) d z^{\prime}\right), \nabla\left(\int_{-H}^{z} \operatorname{div}(\mathbf{v}) d z^{\prime}\right)\right) \\
& +\left(\nu_{1 v} \operatorname{div}(\mathbf{u}), \operatorname{div}(\mathbf{v})\right) .
\end{aligned}
$$


We now define:

$$
\begin{aligned}
& a_{11}(\mathbf{u}, \mathbf{v})=\nu_{1 h}(\nabla \mathbf{u}, \nabla \mathbf{v})+\left(\nu_{1 v} \frac{\partial \mathbf{u}}{\partial z}, \frac{\partial \mathbf{v}}{\partial z}\right) \\
& a_{12}(\mathbf{u}, \mathbf{v})=\nu_{1 h}(\nabla W(\mathbf{u}), \nabla W(\mathbf{v}))+\left(\nu_{1 v} \operatorname{div}(\mathbf{u}), \operatorname{div}(\mathbf{v})\right), \\
& a_{1}(\mathbf{u}, \mathbf{v})=a_{11}(\mathbf{u}, \mathbf{v})+a_{12}(\mathbf{u}, \mathbf{v}) \\
& a_{2}(\rho, \phi)=\nu_{2 h}(\nabla \rho, \nabla \phi)+\left(\nu_{2 v} \frac{\partial \rho}{\partial z}, \frac{\partial \phi}{\partial z}\right) \\
& d(\mathbf{u}, \mathbf{v})=\left(\mathbf{F} \wedge_{2} \mathbf{u}, \mathbf{v}\right) \\
& b_{1}(\mathbf{u}, \mathbf{v}, \mathbf{w})=((\mathbf{u} . \nabla) \mathbf{v}, \mathbf{w})+\left(W(\mathbf{u}) \frac{\partial \mathbf{v}}{\partial z}, \mathbf{w}\right) \\
& b_{2}(\mathbf{u}, \rho, \phi)=((\mathbf{u} \cdot \nabla) \rho, \phi)+\left(W(\mathbf{u}) \frac{\partial \rho}{\partial z}, \phi\right) \\
& l_{1}(\mathbf{u}, \mathbf{v})=b_{1}\left(\mathbf{u}, \mathbf{u}_{\mathbf{0}}, \mathbf{v}\right)+b_{1}\left(\mathbf{u}_{\mathbf{0}}, \mathbf{u}, \mathbf{v}\right) \\
& l_{2}(\mathbf{u}, \rho, \phi)=b_{2}\left(\mathbf{u}, \rho_{0}, \phi\right)+b_{2}\left(\mathbf{u}_{\mathbf{0}}, \rho, \phi\right)
\end{aligned}
$$

Lemma 2.2. Suppose that $\mathbf{f} \in L^{2}\left(0, T, L^{2}\left(\Gamma_{0}\right)\right)$ and that $(\mathbf{u}, \rho, P)$ is a sufficiently regular. Then:

(i) $-\nu_{1 h}(\Delta \mathbf{u}, \mathbf{v})-\left(\frac{\partial}{\partial z}\left(\nu_{1 v} \frac{\partial \mathbf{u}}{\partial z}\right), \mathbf{v}\right)=a_{11}(\mathbf{u}, \mathbf{v})-\left(\mathbf{f}, \gamma_{0} \mathbf{v}\right), \forall \mathbf{v} \in \mathcal{W}_{1}$,

(ii) $-\nu_{2 h}(\Delta \rho, \phi)-\left(\frac{\partial}{\partial z}\left(\nu_{1 v} \frac{\partial \rho}{\partial z}\right), \phi\right)=a_{2}(\rho, \phi), \forall \phi \in \mathcal{V}_{2}$,

(iii) $(\nabla P, \mathbf{v})=-\left(P, \int_{-H}^{0} \operatorname{div}(\mathbf{v}) d z\right)_{\Gamma_{0}}, \forall \mathbf{v} \in \mathcal{W}_{1}$,

(iv) $(\nabla P, \mathbf{v})=0, \forall \mathbf{v} \in \mathcal{V}_{1}$,

(v) $\left(\nabla\left(\int_{z}^{0} \rho d z^{\prime}\right), \mathbf{v}\right)=-\left(\int_{z}^{0} \rho d z^{\prime}, \operatorname{div}(\mathbf{v})\right)=\left(\rho, \int_{-H}^{z} \operatorname{div}(\mathbf{v}) d z^{\prime}\right), \forall \mathbf{v} \in \mathcal{W}_{1}$ where $\gamma_{0} \mathbf{v}$ denotes the trace of $\mathbf{v}$ on $\Gamma_{0}$ and $\left(\mathbf{f}, \gamma_{0} \mathbf{v}\right)_{\Gamma_{0}}$ denotes the scalar product in $L^{2}\left(\Gamma_{0}\right)$, which makes sense if $\mathbf{v} \in \mathcal{W}_{1}$.

Proof. Results (i) and (ii) are deduced from the definition of the spaces $\mathcal{W}_{1}, \mathcal{V}_{1}$ and $\mathcal{V}_{2}$, and from the boundary conditions satisfied by $(\mathbf{u}, \rho)$ on $\Gamma$.

(iii) According to Green's formula and boundary conditions verified by $\mathbf{v} \in$ $\mathcal{W}_{1}$, we then obtain: $(\nabla P, \mathbf{v})=-(P, \operatorname{div}(\mathbf{v})) . P$ is only a function of $x$ and $y$ we can deduce that:

$$
(\nabla P, \mathbf{v})=-\left(P, \int_{-H}^{0} \operatorname{div}(\mathbf{v}) d z^{\prime}\right)_{\Gamma_{0}} .
$$

(iv) If $\mathbf{v} \in \mathcal{V}_{1}$, we have $\int_{-H}^{0} \operatorname{div}(\mathbf{v}) d z^{\prime}=0$ and therefore, $(\nabla P, \mathbf{v})=0$. 
(v) According to Green's formula and boundary conditions verified by $\mathbf{v} \in$ $\mathcal{W}_{1}$, we then have

$$
\left(\nabla\left(\int_{z}^{0} \rho d z^{\prime}\right), \mathbf{v}\right)=-\left(\int_{z}^{0} \rho d z^{\prime}, \operatorname{div}(\mathbf{v})\right) .
$$

By integrating by part with respect to $z$ gives

$$
\left(\nabla\left(\int_{z}^{0} \rho d z^{\prime}\right), \mathbf{v}\right)=\left(\rho, \int_{-H}^{z} \operatorname{div}(\mathbf{v}) d z^{\prime}\right) .
$$

The initial problem $(1.9),(1.5),(1.6)$ satisfied by the perturbation $(\mathbf{u}, \rho, P)$ of the mean flow admits two weak formulations:

$$
\begin{aligned}
& \text { Find }(\mathbf{u}, \rho) \in L^{2}\left(0, T, \mathcal{V}_{1}\right) \times L^{2}\left(0, T, \mathcal{V}_{2}\right) \text { such that } \\
& \left(\frac{\partial \mathbf{u}}{\partial t}, \mathbf{v}\right)+a_{1}(\mathbf{u}, \mathbf{v})+l_{1}(\mathbf{u}, \mathbf{v})+d(\mathbf{u}, \mathbf{v})+\frac{g}{\rho_{\text {moy }}}\left(\rho, \int_{-H}^{z} \operatorname{div}(\mathbf{v}) d z^{\prime}\right) \\
& =\left(\mathbf{f}, \gamma_{0} \mathbf{v}\right)_{\Gamma_{0}}, \forall \mathbf{v} \in \mathcal{V}_{1} \\
& \left(\frac{\partial \rho}{\partial t}, \phi\right)+a_{2}(\rho, \phi)+l_{2}(\mathbf{u}, \rho, \phi)=0, \forall \phi \in \mathcal{V}_{2}, \\
& \mathbf{u}(0)=\mathbf{0}, \rho(0)=0
\end{aligned}
$$

and

Find $(\mathbf{u}, \rho, P) \in L^{2}\left(0, T, \mathcal{W}_{1} \cap H^{2}\right) \times L^{2}\left(0, T, \mathcal{V}_{2} \cap H^{2}\right) \times L^{2}\left(0, T, L^{2}\left(\Gamma_{0}\right)\right)$ such that

$$
\begin{aligned}
& \left(\frac{\partial \mathbf{u}}{\partial t}, \mathbf{v}\right)+a_{1}(\mathbf{u}, \mathbf{v})+l_{1}(\mathbf{u}, \mathbf{v})+d(\mathbf{u}, \mathbf{v})+\frac{g}{\rho_{\text {moy }}}\left(\rho, \int_{-H}^{z} \operatorname{div}(\mathbf{v}) d z^{\prime}\right) \\
& \quad-\left(\nu_{1 v} \gamma_{0}(\operatorname{div}(\mathbf{u})), \int_{-H}^{0} \operatorname{div}(\mathbf{v}) d z^{\prime}\right)_{\Gamma_{0}}-\frac{1}{\rho_{m o y}}\left(P, \int_{-H}^{0} \operatorname{div}(\mathbf{v}) d z^{\prime}\right)_{\Gamma_{0}} \\
& =\left(\mathbf{f}, \gamma_{0} \mathbf{v}\right)_{\Gamma_{0}}, \forall \mathbf{v} \in \mathcal{W}_{1}, \\
& \left(\frac{\partial \rho}{\partial t}, \phi\right)+a_{2}(\rho, \phi)+l_{2}(\mathbf{u}, \rho, \phi)=0, \forall \phi \in \mathcal{V}_{2}, \\
& \left(\int_{-H}^{0} \operatorname{div}(\mathbf{u}) d z^{\prime}, Q\right)_{\Gamma_{0}}=0, \forall Q \in L^{2}\left(\Gamma_{0}\right), \\
& \mathbf{u}(0)=\mathbf{0}, \rho(0)=0 .
\end{aligned}
$$

In the two following propositions we are dealing with some properties of operators $a_{1}, a_{2}, d, l_{1}$ and $l_{2}$.

Proposition 2.1. (i) $a_{1}$ (resp. $a_{2}$ ) is a bilinear continuous and coercive form on $\mathcal{W}_{1}^{2}$ (resp. on $\left.\mathcal{V}_{2}^{2}\right)$,

(ii) $d$ is a bilinear continuous form on $\mathcal{W}_{1}^{2}$, 
(iii) $\|W(\mathbf{u})\|_{L^{2}(\Omega)} \leq c\|\mathbf{u}\|$.

(iv) $\left|\frac{g}{\rho_{\text {moy }}}\left(\rho, \int_{-H}^{z} \operatorname{div}(\mathbf{v}) d z^{\prime}\right)\right| \leq c\|\rho\|_{L^{2}(\Omega)}\|\mathbf{v}\|$, $\leq c\|\mathbf{v}\|_{L^{2}(\Omega)}\|\rho\|, \forall(\mathbf{v}, \rho) \in \mathcal{W}_{1} \times \mathcal{V}_{2}$.

Proof. (i) $\nu_{1 h}$ and $\nu_{2 h}$ are constant coefficients, $\nu_{1 v}$ and $\nu_{2 v}$ are bounded functions, therefore the continuity and the coercivity of $a_{1}$ and $a_{2}$ are easily obtained. The constant of coercivity of $\alpha_{1}$ (resp. $\alpha_{2}$ ) is then given by $\alpha_{1}=\min \left(\nu_{1 h}, \min \left(\nu_{1 v}\right)\right)\left(\right.$ resp. $\left.\alpha_{2}=\min \left(\nu_{2 h}, \min \left(\nu_{2 v}\right)\right)\right)$.

(ii) $d(\mathbf{u}, \mathbf{v})=\left(\mathbf{F} \wedge_{2} \mathbf{u}, \mathbf{v}\right)$, with $\mathbf{F}=(0,0,2 \omega \sin (\phi))$, then

$$
|d(\mathbf{u}, \mathbf{v})| \leq 2 \omega|\mathbf{u}||\mathbf{v}| \leq C\|\mathbf{u}\|\|\mathbf{v}\|
$$

(iii) Since $W(\mathbf{u})=-\int_{-H}^{z} \operatorname{div}(\mathbf{u}) d z^{\prime}$, we have

$$
\|W(\mathbf{u})\|_{L^{2}(\Omega)}^{2} \leq \int_{\Omega}\left(\int_{-H}^{0}|\operatorname{div}(\mathbf{u})| d z^{\prime}\right)^{2} d \Omega
$$

Applying Hölder's inequality, we obtain

$$
\left(\int_{-H}^{0}|\operatorname{div}(\mathbf{u})| d z^{\prime}\right)^{2} \leq H \int_{-H}^{0}|\operatorname{div}(\mathbf{u})|^{2} d z^{\prime} .
$$

Since $\left.\Omega=\Omega_{0} \times\right]-H, 0[$, this implies

$$
\|W(\mathbf{u})\|_{L^{2}(\Omega)}^{2} \leq H \int_{\Omega}\left(\int_{-H}^{0}|\operatorname{div}(\mathbf{u})|^{2} d z^{\prime}\right) d \Omega=H^{2} \int_{\Omega}|\operatorname{div}(\mathbf{u})|^{2} d \Omega,
$$

and then $\|W(\mathbf{u})\|_{L^{2}(\Omega)} \leq c\|\mathbf{u}\|$.

(iv) We have

$$
\begin{aligned}
\left|\frac{g}{\rho_{\text {moy }}}\left(\rho, \int_{-H}^{z} \operatorname{div}(\mathbf{v}) d z^{\prime}\right)\right| & \leq \frac{g}{\rho_{\text {moy }}}\|\rho\|_{L^{2}(\Omega)}\left\|\int_{-H}^{z} \operatorname{div}(\mathbf{v}) d z^{\prime}\right\|_{L^{2}(\Omega)} \\
& =\frac{g}{\rho_{\text {moy }}}\|\rho\|_{L^{2}(\Omega)}\|W(\mathbf{v})\|_{L^{2}(\Omega)}^{2} .
\end{aligned}
$$

By using (iii) we then obtain

$$
\left|\frac{g}{\rho_{\text {moy }}}\left(\rho, \int_{-H}^{z} \operatorname{div}(\mathbf{v}) d z^{\prime}\right)\right| \leq c\|\rho\|_{L^{2}(\Omega)}\|\mathbf{v}\| .
$$

According to Lemma 2.2, we have

$$
\frac{g}{\rho_{\text {moy }}}\left(\rho, \int_{-H}^{z} \operatorname{div}(\mathbf{v}) d z^{\prime}\right)=\frac{g}{\rho_{\text {moy }}}\left(\nabla\left(\int_{z}^{0} \rho d z^{\prime}\right), \mathbf{v}\right) .
$$


So,

$$
\begin{aligned}
& \left|\frac{g}{\rho_{\text {moy }}}\left(\rho, \int_{-H}^{z} \operatorname{div}(\mathbf{v}) d z^{\prime}\right)\right| \leq \frac{g}{\rho_{\text {moy }}} \|\left(\nabla\left(\int_{z}^{0} \rho d z^{\prime}\right)\left\|_{L^{2}(\Omega)}\right\| \mathbf{v} \|_{L^{2}(\Omega)},\right. \\
& \leq \frac{g}{\rho_{\text {moy }}} H\|\nabla \rho\|_{L^{2}(\Omega)}\|\mathbf{v}\|_{L^{2}(\Omega)}, \leq c\|\rho\|\|\mathbf{v}\|_{L^{2}(\Omega)} .
\end{aligned}
$$

Proposition 2.2. If the mean circulation $\left(\mathbf{u}_{\mathbf{0}}, w_{0}, \rho_{0}\right)$ is given such that: $\mathbf{u}_{\mathbf{0}}, w_{0}, \rho_{0}, \nabla \mathbf{u}_{\mathbf{0}}, \frac{\partial \mathbf{u}_{\mathbf{0}}}{\partial z}, \nabla \rho_{0}$ and $\frac{\partial \rho_{0}}{\partial z} \in L^{\infty}(\Omega)$, then there exists a positive constant $c_{0}$ such that

$$
\begin{aligned}
& \left|b_{1}\left(\mathbf{u}_{\mathbf{0}}, \mathbf{u}, \mathbf{v}\right)\right| \leq c_{0}\|\mathbf{u}\|\|\mathbf{v}\|_{L^{2}(\Omega)}, \quad \forall(\mathbf{u}, \mathbf{v}) \in \mathcal{W}_{1}^{2}, \\
& \left|b_{2}\left(\mathbf{u}_{\mathbf{0}}, \rho, \phi\right)\right| \leq c_{0}\|\rho\|\|\phi\|_{L^{2}(\Omega)}, \quad \forall(\rho, \phi) \in \mathcal{V}_{2}^{2}, \\
& \left|b_{1}\left(\mathbf{u}, \mathbf{u}_{\mathbf{0}}, \mathbf{v}\right)\right| \leq c_{0}\|\mathbf{u}\|\|\mathbf{v}\|_{L^{2}(\Omega)}, \quad \forall(\mathbf{u}, \mathbf{v}) \in \mathcal{W}_{1}^{2}, \\
& \left|b_{2}\left(\mathbf{u}, \rho_{0}, \phi\right)\right| \leq c_{0}\|\mathbf{u}\|\|\phi\|_{L^{2}(\Omega)}, \quad \forall(\mathbf{u}, \phi) \in \mathcal{W}_{1} \times \mathcal{V}_{2}, \\
& \left|l_{1}(\mathbf{u}, \mathbf{v})\right| \leq c_{0}\|\mathbf{u}\|\|\mathbf{v}\|_{L^{2}(\Omega)}, \quad \forall(\mathbf{u}, \mathbf{v}) \in \mathcal{W}_{1}^{2}, \\
& \left|l_{2}(\mathbf{u}, \rho, \phi)\right| \leq c_{0}(\|\mathbf{u}\|+\|\rho\|)\|\phi\|_{L^{2}(\Omega)}, \quad \forall(\mathbf{u}, \rho, \phi) \in \mathcal{W}_{1} \times \mathcal{V}_{2}^{2}
\end{aligned}
$$

Proof. We have

$$
\begin{aligned}
& b_{1}\left(\mathbf{u}_{\mathbf{0}}, \mathbf{u}, \mathbf{v}\right)=\left(\left(\mathbf{u}_{\mathbf{0}} \nabla\right) \mathbf{u}, \mathbf{v}\right)+\left(w_{0} \frac{\partial \mathbf{u}}{\partial z}, \mathbf{v}\right) . \\
& \left|\left(\left(\mathbf{u}_{\mathbf{0}} \nabla\right) \mathbf{u}, \mathbf{v}\right)\right| \leq \sum_{i, j=1}^{2} \int_{\Omega}\left|\mathbf{u}_{\mathbf{0} i}\right|\left|\partial_{i} \mathbf{u}_{j}\right|\left|\mathbf{v}_{j}\right| d \Omega .
\end{aligned}
$$

If $\mathbf{u}_{\mathbf{0}} \in L^{\infty}(\Omega)$, there exists a positive constant $c_{0}$ such that

$$
\left|\left(\left(\mathbf{u}_{\mathbf{0}} \nabla\right) \mathbf{u}, \mathbf{v}\right)\right| \leq c_{0}\|\mathbf{u}\|\|\mathbf{v}\|_{L^{2}(\Omega)} .
$$

Since $w_{0} \in L^{\infty}(\Omega)$, we have:

$$
\left|\left(w_{0} \frac{\partial \mathbf{u}}{\partial z}, \mathbf{v}\right)\right| \leq c_{0}\|\mathbf{u}\|\|\mathbf{v}\|_{L^{2}(\Omega)} .
$$

We then obtain

$$
\left|b_{1}\left(\mathbf{u}_{\mathbf{0}}, \mathbf{u}, \mathbf{v}\right)\right| \leq c_{0}\|\mathbf{u}\|\|\mathbf{v}\|_{L^{2}(\Omega)} .
$$

The same proof is valid for the majoration of $\left|b_{2}\left(\mathbf{u}_{\mathbf{0}}, \rho, \phi\right)\right|$.

$$
\begin{aligned}
& b_{1}\left(\mathbf{u}, \mathbf{u}_{\mathbf{0}}, \mathbf{v}\right)=\left((\mathbf{u} \nabla) \mathbf{u}_{\mathbf{0}}, \mathbf{v}\right)+\left(W(\mathbf{u}) \frac{\partial \mathbf{u}_{\mathbf{0}}}{\partial z}, \mathbf{v}\right) \\
& \left|\left(\left(\mathbf{u}_{\mathbf{0}} \nabla\right) \mathbf{u}, \mathbf{v}\right)\right| \leq \sum_{i, j=1}^{2} \int_{\Omega}\left|\mathbf{u}_{i}\right|\left|\partial_{i} \mathbf{u}_{\mathbf{0}_{j}} \| \mathbf{v}_{j}\right| d \Omega
\end{aligned}
$$


Since $\nabla \mathbf{u}_{\mathbf{0}} \in L^{\infty}(\Omega)$, we have the majoration:

$$
\left|\left((\mathbf{u} \nabla) \mathbf{u}_{\mathbf{0}}, \mathbf{v}\right)\right| \leq c_{0}\|\mathbf{u}\|\|\mathbf{v}\|_{L^{2}(\Omega)} .
$$

Since $\frac{\partial \mathbf{u}_{\mathbf{0}}}{\partial z} \in L^{\infty}(\Omega)$, we have

$$
\left|\left(W(\mathbf{u}) \frac{\partial \mathbf{u}_{\mathbf{0}}}{\partial z}, \mathbf{v}\right)\right| \leq c_{0}\|W(\mathbf{u})\|_{L^{2}(\Omega)}\|\mathbf{v}\|_{L^{2}(\Omega)} .
$$

By applying Proposition 2.1, we obtain

$$
\left|\left(W(\mathbf{u}) \frac{\partial \mathbf{u}_{\mathbf{0}}}{\partial z}, \mathbf{v}\right)\right| \leq c_{0}\|\mathbf{u}\|\|\mathbf{v}\|_{L^{2}(\Omega)} .
$$

We can now conclude that

$$
\left|b_{1}\left(\mathbf{u}, \mathbf{u}_{\mathbf{0}}, \mathbf{v}\right)\right| \leq c_{0}\|\mathbf{u}\|\|\mathbf{v}\|_{L^{2}(\Omega)} .
$$

The same proof is valid for the majoration of $\left|b_{2}\left(\mathbf{u}, \rho_{0}, \phi\right)\right|$. Since $l_{1}(\mathbf{u}, \mathbf{v})=$ $b_{1}\left(\mathbf{u}_{\mathbf{0}}, \mathbf{u}, \mathbf{v}\right)+b_{1}\left(\mathbf{u}, \mathbf{u}_{\mathbf{0}}, \mathbf{v}\right)$ and $l_{2}(\mathbf{u}, \rho, \phi)=b_{2}\left(\mathbf{u}_{\mathbf{0}}, \rho, \phi\right)+b_{2}\left(\mathbf{u}, \rho_{0}, \phi\right)$, by using the majorations of $b_{1}$ and $b_{2}$ we obtain the majorations of $\left|l_{1}(\mathbf{u}, \mathbf{v})\right|$ and $\left|l_{2}(\mathbf{u}, \rho, \phi)\right|$.

We are now going to prove the existence and uniqueness of a solution.

\subsection{Existence and uniqueness of the solution.}

Theorem 2.1. For $\mathbf{f}, \mathbf{u}_{\mathbf{0}}, w_{0}$ and $\rho_{0}$ given such that: $\mathbf{f} \in L^{2}\left(0, T, L^{2}\left(\Gamma_{0}\right)\right)$, $\mathbf{u}_{\mathbf{0}}, w_{0}, \rho_{0}, \nabla \mathbf{u}_{\mathbf{0}}, \frac{\partial \mathbf{u}_{\mathbf{0}}}{\partial z}, \nabla \rho_{0}$ and $\frac{\partial \rho_{0}}{\partial z} \in L^{\infty}(\Omega)$, there exists a unique solution $(\mathbf{u}, \rho)$ of problem $(2.1)$ verifying:

$$
\begin{aligned}
& \mathbf{u} \in L^{2}\left(0, T, \mathcal{V}_{1}\right) \cap C^{0}\left([0, T], \mathcal{H}_{1}\right), \frac{\partial \mathbf{u}}{\partial t} \in L^{2}\left(0, T, \mathcal{V}_{1}^{\prime}\right) \\
& \rho \in L^{2}\left(0, T, \mathcal{V}_{2}\right) \cap C^{0}\left([0, T], \mathcal{H}_{2}\right), \frac{\partial \rho}{\partial t} \in L^{2}\left(0, T, \mathcal{V}_{2}^{\prime}\right)
\end{aligned}
$$

Proof. To prove the existence of a solution we use the Faedo-Galerkin method (see [25], for example). Set $\left(\mathbf{v}_{1}, \ldots, \mathbf{v}_{l}, \ldots\right)$ a total and free sequence in $\mathcal{V}_{1}$ and $\left(\phi_{1}, \ldots, \phi_{l}, \ldots\right)$ a total and free sequence in $\mathcal{V}_{2}$ (see [25, p. 255]). Set $\left(\mathbf{u}_{l}, \rho_{l}\right)=\sum_{i=1}^{l} g_{i l}(t)\left(\mathbf{v}_{i}, \phi_{i}\right)$ (the function $g_{i l}$ are scalar functions defined on $[0, T])$ an approximation solution of problem (2.1), verifying the following 
problem:

$$
\begin{aligned}
& \left(\frac{\partial \mathbf{u}_{l}}{\partial t}, \mathbf{v}_{k}\right)+a_{1}\left(\mathbf{u}_{l}, \mathbf{v}_{k}\right)+l_{1}\left(\mathbf{u}_{l}, \mathbf{v}_{k}\right) \\
& \quad+d\left(\mathbf{u}_{l}, \mathbf{v}_{k}\right)+\frac{g}{\rho_{\text {moy }}}\left(\rho_{l}, \int_{-H}^{z} \operatorname{div}\left(\mathbf{v}_{k}\right) d z^{\prime}\right)=\left(\mathbf{f}, \gamma_{0} \mathbf{v}_{k}\right)_{\Gamma_{0}}, \\
& \left(\frac{\partial \rho_{l}}{\partial t}, \phi_{k}\right)+a_{2}\left(\rho_{l}, \phi_{k}\right)+l_{2}\left(\mathbf{u}_{l}, \rho_{l}, \phi_{k}\right)=0, \forall k=1, \ldots, l, \\
& \mathbf{u}_{l}(t=0)=\mathbf{0}, \rho_{l}(t=0)=0 .
\end{aligned}
$$

The existence and uniqueness of the approached solution $\left(\mathbf{u}_{l}, \rho_{l}\right)$ are easily obtained. In order to extract a converging subsequence from $\left(\mathbf{u}_{l}, \rho_{l}\right)$, we have to prove that the sequence $\left(\mathbf{u}_{l}, \rho_{l}\right)$ is bounded in $L^{\infty}\left(0, T, \mathcal{H}_{1} \times \mathcal{H}_{2}\right) \cap$ $L^{2}\left(0, T, \mathcal{V}_{1} \times \mathcal{V}_{2}\right)$

By applying the coercivity of $a_{1}$ and $a_{2}$, the result of Propositions 2.1 and 2.2 we obtain the estimations:

$$
\begin{aligned}
& \frac{d}{d t}\left|\mathbf{u}_{l}\right|^{2}+2 \alpha_{1}\left\|\mathbf{u}_{l}\right\|_{W}^{2} \leq c_{1}\left|\mathbf{u}_{l}\right|\left\|\mathbf{u}_{l}\right\|+c_{2}\left|\rho_{l}\right|\left\|\mathbf{u}_{l}\right\|+c_{3}|\mathbf{f}|_{\Gamma_{0}}\left\|\mathbf{u}_{l}\right\|, \\
& \frac{d}{d t}\left|\rho_{l}\right|^{2}+2 \alpha_{2}\left\|\rho_{l}\right\|^{2} \leq c_{4}\left|\rho_{l}\right|\left\|\mathbf{u}_{l}\right\|+c_{5}\left|\rho_{l}\right|\left\|\rho_{l}\right\|,
\end{aligned}
$$

where $\left(c_{i}\right)_{i=1, \ldots, 5}$ are positive constants.

By applying Hölder's inequality, we deduce from (2.4) that there exists a positive constants $\left(\varepsilon_{i}\right)_{i=1, \ldots, 3}$ as small as wanted such that:

$$
\begin{aligned}
& \frac{d}{d t}\left|\mathbf{u}_{l}\right|^{2}+\left(2 \alpha_{1}-\varepsilon_{1}\right)\left\|\mathbf{u}_{l}\right\|_{W}^{2} \leq c_{6}\left|\mathbf{u}_{l}\right|^{2}+c_{7}\left|\rho_{l}\right|^{2}+c_{8}|\mathbf{f}|_{\Gamma_{0}}^{2}, \\
& \frac{d}{d t}\left|\rho_{l}\right|^{2}+\left(2 \alpha_{2}-\varepsilon_{2}\right)\left\|\rho_{l}\right\|^{2} \leq c_{9}\left|\rho_{l}\right|^{2}+\varepsilon_{3}\|\mathbf{u}\|_{W}^{2} .
\end{aligned}
$$

We choose $\left(\varepsilon_{i}\right)_{i=1, \ldots, 3}$ in order to have $2 \alpha_{1}-\varepsilon_{1}-\varepsilon_{3}>0$ and $2 \alpha_{2}-\varepsilon_{2}>0$. By setting $K=\min \left(2 \alpha_{1}-\varepsilon_{1}-\varepsilon_{3}, 2 \alpha_{2}-\varepsilon_{2}\right)$, and $X_{l}=\left(\mathbf{u}_{l}, \rho_{l}\right)$, we so obtain

$$
\frac{d}{d t}\left|X_{l}\right|^{2}+K\left\|X_{l}\right\|_{W}^{2} \leq c_{10}\left|X_{l}\right|^{2}+c_{11}|\mathbf{f}|_{\Gamma_{0}}^{2},
$$

where, $\left|X_{l}\right|^{2}=\left|\mathbf{u}_{l}\right|^{2}+\left|\rho_{l}\right|^{2}$, and $\left\|X_{l}\right\|_{W}^{2}=\left\|\mathbf{u}_{l}\right\|_{W}^{2}+\left\|\rho_{l}\right\|^{2}$.

Applying the Gronwall lemma, we easily deduce from (2.6) that the sequence $X_{l}$ is bounded in $L^{\infty}\left(0, T, \mathcal{H}_{1} \times \mathcal{H}_{2}\right) \cap L^{2}\left(0, T, \mathcal{V}_{1} \times \mathcal{V}_{2}\right)$. This result makes it possible to extract from $X_{l}$ a subsequence converging towards $(\mathbf{u}, \rho)$ weakly star in $L^{\infty}\left(0, T, \mathcal{H}_{1} \times \mathcal{H}_{2}\right)$, weakly in $L^{2}\left(0, T, \mathcal{V}_{1} \times \mathcal{V}_{2}\right)$.

It is then easy to prove that $(\mathbf{u}, \rho)$ is the unique solution of (2.1) and verifies the regularity:

$$
(\mathbf{u}, \rho) \text { in } L^{2}\left(0, T, \mathcal{V}_{1} \times \mathcal{V}_{2}\right) \text {, where }\left(\frac{\partial \mathbf{u}}{\partial t}, \frac{\partial \rho}{\partial t}\right) \in L^{2}\left(0, T, \mathcal{V}_{1}^{\prime} \times \mathcal{V}_{2}^{\prime}\right)
$$

By Lions [18] we conclude that $(\mathbf{u}, \rho) \in C^{0}\left([0, T], \mathcal{H}_{1} \times \mathcal{H}_{2}\right)$. 
Proposition 2.3. If $(\mathbf{u}, \rho)$ is the solution of problem (2.1), then there is a unique distribution $P$ (up to a constant) such that $(\mathbf{u}, \rho, P)$ is solution of problem (1.9), in the distribution sense.

Proof. Using the result proved by Lions-Temam-Wang [20, Lemma 2.2, p. 1025] and Lions-Temam-Wang [19, pp. 266-267] we have the existence of surface pressure $P$ such that $(\mathbf{u}, \rho, P)$ is a solution of problem (1.9), in the distribution sense.

Remark 2.3. $\left(\mathbf{u}_{\mathbf{0}}, w_{0}, \rho_{0}\right)$ is given and has to represent the mean circulation in a tropical area. For any physically significant circulation $\mathbf{u}_{\mathbf{0}}, \nabla \mathbf{u}_{\mathbf{0}}, \rho_{0}$, $\frac{\partial w_{0}}{\partial z}, \nabla \rho_{0}$ and $\frac{\partial \rho_{0}}{\partial z}$ are bounded in $\Omega$. Therefore the conditions imposed to $\left(\mathbf{u}_{\mathbf{0}}, w_{0}, \rho_{0}\right)$ are not restrictive.

\subsection{A result of regularity.}

The regularity of the solution $(\mathbf{u}, \rho, P)$ depends on the regularity of the wind-stress $\mathbf{f}$. We are going to give sufficient conditions about the windstress $\mathbf{f}$ in order to define this quantity even near the corners of the open set $\Omega$.

From now on we assume the following regularity of the wind-stress:

$$
\mathbf{f} \in L^{2}\left(0, T, H^{1}\left(\Gamma_{0}\right)\right), \frac{\partial \mathbf{f}}{\partial t} \in L^{2}\left(0, T, L^{2}\left(\Gamma_{0}\right)\right) .
$$

Remark 2.4. (2.7) implies that $\mathbf{f} \in \mathcal{C}^{0}\left([0, T], L^{2}\left(\Gamma_{0}\right)\right)$ a.e on $[0, T]$.

f must be consistent with the initial and boundary conditions (1.5), (1.6) imposed to the velocity $\mathbf{u}$. We then have to impose the following compatibility conditions:

$$
\begin{aligned}
& \mathbf{f}(0)=\mathbf{0} \text { on } \Gamma_{0}, \\
& f_{2}=0 \text { and } \frac{\partial f_{1}}{\partial y}=0 \text { on } \gamma_{3} \cup \gamma_{4}, \\
& \mathbf{f}_{\left.\right|_{\gamma_{1}}}=\mathbf{f}_{\left.\right|_{\gamma_{2}}} .
\end{aligned}
$$

Notation: $\mathbf{f}$ is defined on the open set $\left.\Gamma_{0}=\right] 0, L_{x}[\times]-L_{y}, L_{y}[, \gamma$ denotes the boundary of $\Gamma_{0} ; \gamma=\bigcup_{i=1, \ldots, 4} \gamma_{i}$, where $\gamma_{1}$ and $\gamma_{2}$ are the western and eastern boundaries, $\gamma_{3}$ and $\gamma_{4}$ the southern and northern boundaries. 
Lemma 2.3. If the wind-stress $\mathbf{f}$ satisfies the conditions (2.7), (2.8), then $(\mathbf{u}, \rho)$, solution of problem $(2.1)$, is such that:

$$
\begin{aligned}
& (\mathbf{u}, \rho) \in \mathcal{C}^{0}\left([0, T], \mathcal{V}_{1} \times \mathcal{V}_{2}\right) \\
& \left(\frac{\partial \mathbf{u}}{\partial t}, \frac{\partial \rho}{\partial t}\right) \in L^{2}\left(0, T, \mathcal{V}_{1} \times \mathcal{V}_{2}\right) \cap \mathcal{C}^{0}\left([0, T], \mathcal{H}_{1} \times \mathcal{H}_{2}\right)
\end{aligned}
$$

Proof. Introduce the following problem:

Find $(\mathbf{w}, \Psi)$ such that:

$$
\begin{aligned}
& \left(\frac{\partial \mathbf{w}}{\partial t}, \mathbf{v}\right)+a_{1}(\mathbf{w}, \mathbf{v})+l_{1}(\mathbf{w}, \mathbf{v})+d(\mathbf{w}, \mathbf{v})+\frac{g}{\rho_{m o y}}\left(\Psi, \int_{-H}^{z} \operatorname{div}(\mathbf{v}) d z^{\prime}\right) \\
& =\left(\frac{\partial \mathbf{f}}{\partial t}, \gamma_{0} \mathbf{v}\right)_{\Gamma_{0}}, \forall \mathbf{v} \in \mathcal{V}_{1}, \\
& \left(\frac{\partial \Psi}{\partial t}, \phi\right)+a_{2}(\Psi, \phi)+l_{2}(\mathbf{w}, \Psi, \phi)=0, \forall \phi \in \mathcal{V}_{2}, \\
& \mathbf{w}(0)=\mathbf{0}, \Psi(0)=0 .
\end{aligned}
$$

According to Theorem 2.1, problem (2.9) admits one unique solution (w, $\Psi)$, such that:

$$
\begin{aligned}
& (\mathbf{w}, \Psi) \in L^{2}\left(0, T, \mathcal{V}_{1} \times \mathcal{V}_{2}\right) \cap \mathcal{C}^{0}\left([0, T], \mathcal{H}_{1} \times \mathcal{H}_{2}\right) \\
& \left(\frac{\partial \mathbf{w}}{\partial t}, \frac{\partial \Psi}{\partial t}\right) \in L^{2}\left(0, T, \mathcal{V}_{1}^{\prime} \times \mathcal{V}_{2}^{\prime}\right)
\end{aligned}
$$

Set

$$
\omega(t)=\int_{0}^{t} \mathbf{w}(s) d s \text { and } \psi(t)=\int_{0}^{t} \Psi(s) d s, \quad \forall t \in[0, T]
$$

where $(\omega, \psi) \in \mathcal{C}^{1}\left([0, T], \mathcal{H}_{1} \times \mathcal{H}_{2}\right)$ since $(\mathbf{w}, \Psi) \in \mathcal{C}^{0}\left([0, T], \mathcal{H}_{1} \times \mathcal{H}_{2}\right)$.

According to (2.8),

$$
\mathbf{f}(t)=\int_{0}^{t} \frac{\partial \mathbf{f}}{\partial t}(s) d s
$$

Integrating equations (2.9) with respect to time gives:

$$
\begin{aligned}
& \left(\frac{\partial \omega}{\partial t}, \mathbf{v}\right)+a_{1}(\omega, \mathbf{v})+l_{1}(\omega, \mathbf{v})+d(\omega, \mathbf{v})+\frac{g}{\rho_{\text {moy }}}\left(\psi, \int_{-H}^{z} \operatorname{div}(\mathbf{v}) d z^{\prime}\right) \\
& =\left(\mathbf{f}, \gamma_{0} \mathbf{v}\right)_{\Gamma_{0}}, \forall \mathbf{v} \in \mathcal{V}_{1}, \\
& \left(\frac{\partial \psi}{\partial t}, \phi\right)+a_{2}(\psi, \phi)+l_{2}(\omega, \psi, \phi)=0, \forall \phi \in \mathcal{V}_{2}, \\
& \omega(0)=\mathbf{0}, \psi(0)=0 .
\end{aligned}
$$

We have proved that $(\omega, \psi)$ is a solution of problem (2.1). We then have

$$
(\mathbf{u}, \rho)=(\omega, \psi),(\mathbf{u}, \rho) \in L^{2}\left(0, T, \mathcal{V}_{1} \times \mathcal{V}_{2}\right) \cap \mathcal{C}^{0}\left([0, T], \mathcal{H}_{1} \times \mathcal{H}_{2}\right)
$$


On the other hand,

$$
\left(\frac{\partial \mathbf{u}}{\partial t}, \frac{\partial \rho}{\partial t}\right)=\left(\frac{\partial \omega}{\partial t}, \frac{\partial \psi}{\partial t}\right)=(\mathbf{w}, \Psi)
$$

Therefore,

$$
\left(\frac{\partial \mathbf{u}}{\partial t}, \frac{\partial \rho}{\partial t}\right) \in L^{2}\left(0, T, \mathcal{V}_{1} \times \mathcal{V}_{2}\right) \cap \mathcal{C}^{0}\left([0, T], \mathcal{H}_{1} \times \mathcal{H}_{2}\right)
$$

Since

$$
(\mathbf{u}, \rho) \in L^{2}\left(0, T, \mathcal{V}_{1} \times \mathcal{V}_{2}\right) \text { and }\left(\frac{\partial \mathbf{u}}{\partial t}, \frac{\partial \rho}{\partial t}\right) \in L^{2}\left(0, T, \mathcal{V}_{1} \times \mathcal{V}_{2}\right),
$$

we conclude $([18])$ that $(\mathbf{u}, \rho) \in \mathcal{C}^{0}\left([0, T], \mathcal{V}_{1} \times \mathcal{V}_{2}\right)$.

Theorem 2.2. If the wind-stress $\mathbf{f}$ satisfies the regularity (2.7), and the initial and boundary conditions (2.8), then the solution $(\mathbf{u}, \rho, P)$ of problem (2.1) is such that:

$$
\begin{aligned}
& \mathbf{u} \in L^{2}\left(0, T, H^{2}(\Omega)\right), W(\mathbf{u}) \in L^{2}\left(0, T, H^{2}(\Omega)\right), \\
& \rho \in L^{2}\left(0, T, H^{2}(\Omega)\right), \quad P \in L^{2}\left(0, T, L^{2}\left(\Gamma_{0}\right)\right) .
\end{aligned}
$$

Proof. According to Section 2.2, the solution $(\mathbf{u}, \rho, P)$ of the weak problem satisfies the equations (1.9), which can also be written:

$$
\begin{aligned}
& -\nu_{1 h} \Delta \mathbf{u}-\frac{\partial}{\partial z}\left(\nu_{1 v} \frac{\partial \mathbf{u}}{\partial z}\right)+\frac{1}{\rho_{\text {moy }}} \nabla P+\nabla \mathcal{L}(\mathbf{u}) \\
& =-\left(\frac{\partial \mathbf{u}}{\partial t}+\left(\mathbf{u}_{\mathbf{0}} \cdot \nabla\right) \mathbf{u}+w_{0} \frac{\partial \mathbf{u}}{\partial z}+(\mathbf{u} \cdot \nabla) \mathbf{u}_{\mathbf{0}}+W(\mathbf{u}) \frac{\partial \mathbf{u}_{\mathbf{0}}}{\partial z}+\mathbf{F} \wedge_{2} \mathbf{u}\right. \\
& \left.+\frac{g}{\rho_{\text {moy }}} \nabla R(\rho)\right), \\
& -\nu_{2 h} \Delta \rho-\frac{\partial}{\partial z}\left(\nu_{2 v} \frac{\partial \rho}{\partial z}\right)=-\left(\frac{\partial \rho}{\partial t}+(\mathbf{u} . \nabla) \rho_{0}+W(\mathbf{u}) \frac{\partial \rho_{0}}{\partial z}+\left(\mathbf{u}_{\mathbf{0}} \cdot \nabla\right) \rho+w_{0} \frac{\partial \rho}{\partial z}\right), \\
& \operatorname{div}\left(\int_{-H}^{0} \mathbf{u}\left(z^{\prime}\right) d z^{\prime}\right)=0 .
\end{aligned}
$$

Set

$$
\begin{aligned}
& \mathbf{D}=-\left(\frac{\partial \mathbf{u}}{\partial t}+\left(\mathbf{u}_{\mathbf{0}} \cdot \nabla\right) \mathbf{u}+w_{0} \frac{\partial \mathbf{u}}{\partial z}+(\mathbf{u} . \nabla) \mathbf{u}_{\mathbf{0}}+W(\mathbf{u}) \frac{\partial \mathbf{u}_{\mathbf{0}}}{\partial z}+\mathbf{F} \wedge_{2} \mathbf{u}\right), \\
& d=\frac{g}{\rho_{\text {moy }}} \rho
\end{aligned}
$$

and

$$
\Psi=-\left(\frac{\partial \rho}{\partial t}+(\mathbf{u} \cdot \nabla) \rho_{0}+W(\mathbf{u}) \frac{\partial \rho_{0}}{\partial z}+\left(\mathbf{u}_{\mathbf{0}} \cdot \nabla\right) \rho+w_{0} \frac{\partial \rho}{\partial z}\right)
$$


We define

$$
\frac{p}{\rho_{\text {moy }}}=\frac{P}{\rho_{\text {moy }}}+\frac{g}{\rho_{\text {moy }}} \int_{z}^{0} \rho d z^{\prime}+\int_{z}^{0} L(\mathbf{u}) d z^{\prime}
$$

we can then obtain that:

$$
\begin{aligned}
& -\nu_{1 h} \Delta \mathbf{u}-\frac{\partial}{\partial z}\left(\nu_{1 v} \frac{\partial \mathbf{u}}{\partial z}\right)+\frac{1}{\rho_{\text {moy }}} \nabla p=\mathbf{D}, \\
& -\nu_{1 h} \Delta W(\mathbf{u})-\frac{\partial}{\partial z}\left(\nu_{1 v} \frac{\partial W(\mathbf{u})}{\partial z}\right)+\frac{1}{\rho_{\text {moy }}} \frac{\partial p}{\partial z}=d, \\
& -\nu_{2 h} \Delta \rho-\frac{\partial}{\partial z}\left(\nu_{2 v} \frac{\partial \rho}{\partial z}\right)=\Psi, \\
& \operatorname{div}(\mathbf{u})+\frac{\partial W(\mathbf{u})}{\partial z}=0,
\end{aligned}
$$

If $\mathbf{u}_{\mathbf{0}}, w_{0}$ and $\rho_{0}$ are sufficiently regular, by the result of Lemma 2.3 we have: $(\mathbf{D}, d, \Psi) \in \mathcal{C}^{0}\left([0, T], L^{2}(\Omega)\right)$. At each time $t \in[0, T]$, the initial problem (2.1) can then be written:

$$
\begin{aligned}
& \text { Given }(\mathbf{D}, d, \Psi) \in L^{2}(\Omega), \text { find }(\mathbf{u}, \rho, p) \text { such that }: \\
& -\nu_{1 h} \Delta \mathbf{u}-\frac{\partial}{\partial z}\left(\nu_{1 v} \frac{\partial \mathbf{u}}{\partial z}\right)+\frac{1}{\rho_{\text {moy }}} \nabla p=\mathbf{D}, \text { in } \Omega \\
& -\nu_{1 h} \Delta W(\mathbf{u})-\frac{\partial}{\partial z}\left(\nu_{1 v} \frac{\partial W(\mathbf{u})}{\partial z}\right)+\frac{1}{\rho_{\text {moy }}} \frac{\partial p}{\partial z}=d, \text { in } \Omega \\
& -\nu_{2 h} \Delta \rho-\frac{\partial}{\partial z}\left(\nu_{2 v} \frac{\partial \rho}{\partial z}\right)=\Psi, \text { in } \Omega \\
& \operatorname{div}(\mathbf{u})+\frac{\partial W(\mathbf{u})}{\partial z}=0, \text { in } \Omega
\end{aligned}
$$

and satisfying the boundary conditions (1.5).

At each time $t \in[0, T]$, we then have the regularity result: $(\mathbf{u}, W(\mathbf{u})) \in$ $H^{2}(\Omega), \rho \in H^{2}(\Omega), p \in H^{1}(\Omega)$, except near the corners of the open set $\Omega$ $([2])$. The problem is now to prove the regularity of $(\mathbf{u}, W(\mathbf{u}), \rho, p)$ in the corners.

The solution $(\mathbf{u}, \rho, p)$ is periodic in the $x$-direction. So, the open set $\Omega$ can be extended from $x=-L_{x}$ to $x=2 L_{x}$, and the regularity near the western and eastern boundaries $\Gamma_{1}, \Gamma_{2}$ is automatically obtained.

The open set $\Omega$ can be defined as: $] 0, L_{x}[\times] 0,1[\times]-H, 0[$ by a change of the variable $y$.

To prove the regularity of $(\mathbf{u}, W(\mathbf{u}), \rho, p)$ in the corners (1) and (2), we define $(\tilde{\mathbf{u}}, \tilde{\rho}, \tilde{p})$, extension of $(\mathbf{u}, \rho, p)$ in $\tilde{\Omega}=] 0, L_{x}[\times]-1,1[\times]-H, 0[$.

Set $\tilde{\mathbf{u}}=\mathbf{u}, \tilde{\rho}=\rho, \tilde{p}=p, \tilde{\mathbf{u}}_{\mathbf{0}}=\mathbf{u}_{\mathbf{0}}, \tilde{w}_{0}=w_{0}$ and $\tilde{\rho}_{0}=\rho_{0}$, in $\Omega$. $\tilde{\mathbf{u}}_{i}$ (resp. $\left.\mathbf{u}_{i}\right)$ denotes the components of $\tilde{\mathbf{u}}($ resp. $\mathbf{u})$ and $\left(\tilde{u}_{0}, \tilde{v}_{0}\right)$ (resp. $\left.\left(u_{0}, v_{0}\right)\right)$ denotes the components of $\tilde{\mathbf{u}}_{\mathbf{0}}\left(\right.$ resp. $\left.\mathbf{u}_{\mathbf{0}}\right)$. 
$\Gamma_{0}$

$\Omega_{1}$

$\Omega$

$\Omega_{2}$

$\Gamma_{3} \quad \Gamma_{4}$

FiguRE 1. Extension of the open set $\Omega$

Let $M(x, y, z)$ be a point in $\Omega . \quad M_{1}(x,-y, z)$ denotes its symmetrical about $\Gamma_{3}$. $\tilde{\mathbf{u}}, \tilde{\rho}, \tilde{p}, \tilde{\mathbf{u}}_{\mathbf{0}}, \tilde{w}_{0}$ and $\tilde{\rho}_{0}$ are defined in $\Omega_{1}$ by:

$$
\begin{array}{rlrl}
\tilde{u}_{1}\left(M_{1}\right) & =u_{1}(M), \tilde{u}_{2}\left(M_{1}\right)=-u_{2}(M), W(\tilde{\mathbf{u}})\left(M_{1}\right) & =W(\mathbf{u})(M), \\
\tilde{u}_{0}\left(M_{1}\right) & =u_{0}(M), \tilde{v}_{0}\left(M_{1}\right)=-v_{0}(M), & \tilde{w}_{0}\left(M_{1}\right)=w_{0}(M), \\
\tilde{\rho}\left(M_{1}\right) & =\rho(M), \quad \tilde{\rho}_{0}\left(M_{1}\right)=\rho_{0}(M), & \tilde{p}\left(M_{1}\right) & =p(M) .
\end{array}
$$

$(\tilde{\mathbf{D}}, \tilde{d})$, extension of $(\mathbf{D}, d)$, is defined by:

$$
\begin{aligned}
& \tilde{\mathbf{D}}=\mathbf{D}, \tilde{d}=d \text { in } \Omega \\
& \tilde{D}_{1}\left(M_{1}\right)=D_{1}(M), \tilde{D}_{2}\left(M_{1}\right)=-D_{2}(M), \tilde{d}\left(M_{1}\right)=d(M) .
\end{aligned}
$$

$\tilde{\Psi}$, extension of $\Psi$, is defined by:

$$
\begin{aligned}
& \tilde{\Psi}=\Psi \text { in } \Omega, \\
& \tilde{\Psi}\left(M_{1}\right)=\Psi(M) .
\end{aligned}
$$

$\tilde{\mathbf{f}}$ denotes the extension of the wind-stress on the surface $] 0, L_{x}[\times]-1,1[$, and is defined by:

$$
\begin{aligned}
& \tilde{\mathbf{f}}=\mathbf{f} \text { in } \Gamma_{0}, \\
& \tilde{f}_{1}\left(m_{1}\right)=f_{1}(m), \tilde{f}_{2}\left(m_{1}\right)=-f_{2}(m),
\end{aligned}
$$

where $m(x, y)$ is a point in $\Gamma_{0}$, and $m_{1}(x,-y)$ its symmetrical about $\gamma_{3}$.

The eddy viscosity and diffusivity coefficients are defined in $\Omega_{1}$ by

$$
\tilde{\nu_{1 h}}=\nu_{1 h}, \tilde{\nu_{2} h}=\nu_{2 h}, \tilde{\nu_{1 v}}\left(M_{1}\right)=\nu_{1 v}(M), \tilde{\nu_{2 v}}\left(M_{1}\right)=\nu_{2 v}(M) .
$$


If $(\mathbf{u}, \rho, p)$ is solution of problem $(2.11)$, it is easy to prove that $(\tilde{\mathbf{u}}, \tilde{\rho}, \tilde{p})$ satisfies the equations:

$$
\begin{aligned}
& -\nu_{1 h} \Delta \tilde{\mathbf{u}}-\frac{\partial}{\partial z}\left(\nu_{1 v} \frac{\partial \tilde{\mathbf{u}}}{\partial z}\right)+\frac{1}{\rho_{\text {moy }}} \nabla \tilde{p}=\tilde{\mathbf{D}}, \text { in } \tilde{\Omega} \\
& -\nu_{1 h} \Delta W(\tilde{\mathbf{u}})-\frac{\partial}{\partial z}\left(\nu_{1 v} \frac{\partial W(\tilde{\mathbf{u}})}{\partial z}\right)+\frac{1}{\rho_{m o y}} \frac{\partial \tilde{p}}{\partial z}=\tilde{d}, \text { in } \tilde{\Omega} \\
& -\nu_{2 h} \Delta \tilde{\rho}-\frac{\partial}{\partial z}\left(\nu_{2 v} \frac{\partial \tilde{\rho}}{\partial z}\right)=\tilde{\Psi}, \text { in } \tilde{\Omega} \\
& \operatorname{div}(\tilde{\mathbf{u}})+\frac{\partial W(\tilde{\mathbf{u}})}{\partial z}=0, \text { in } \tilde{\Omega}
\end{aligned}
$$

and boundary conditions similar to conditions (1.5).

$(\tilde{\mathbf{D}}, \tilde{d}, \tilde{\Psi})$ is given in $L^{2}(\tilde{\Omega})$, and we can apply the regularity result: $\tilde{\mathbf{u}} \in$ $H^{2}(\tilde{\Omega}), \quad W(\tilde{\mathbf{u}}) \in H^{2}(\tilde{\Omega}), \tilde{\rho} \in H^{2}(\tilde{\Omega}), \tilde{p} \in H^{1}(\tilde{\Omega})$, except near the corners of $\tilde{\Omega}$. This implies the regularity of $(\mathbf{u}, \rho, p)$ near the corners $(1)$ and $(2)$. To obtain the regularity near the corners (3) and (4), we have to extend the solution $(\mathbf{u}, W(\mathbf{u}), \rho, p)$ in $\Omega_{2}$.

This extension method also allows us to apply the estimate proved in [2], and valid in a sufficiently regular open set. We thus obtain

$$
\begin{aligned}
& \|\mathbf{u}\|_{H^{2}(\Omega)}+\|W(\mathbf{u})\|_{H^{2}(\Omega)}+\|p\|_{H^{1}(\Omega)} \\
& \leq C\left(\|\mathbf{f}\|_{H^{1}\left(\Gamma_{0}\right)}+\|\mathbf{D}\|_{L^{2}(\Omega)}+\|d\|_{L^{2}(\Omega)}\right), \\
& \|\rho\|_{H^{2}(\Omega)} \leq C\|\Psi\|_{L^{2}(\Omega)}, \text { a.e. on }[0, T],
\end{aligned}
$$

where $C$ is a positive constant, independent of time.

We deduce from (2.12) that

$$
\begin{aligned}
& (\mathbf{u}, W(\mathbf{u})) \in L^{2}\left(0, T, H^{2}(\Omega)\right), \rho \in L^{2}\left(0, T, H^{2}(\Omega)\right) \text { and } \\
& P \in L^{2}\left(0, T, L^{2}\left(\Gamma_{0}\right)\right) .
\end{aligned}
$$

Proposition 2.4. If the wind-stress $\mathbf{f}$ satisfies the conditions (2.7), (2.8) and $\mathbf{u}_{\mathbf{0}}, w_{0}, \nabla \mathbf{u}_{\mathbf{0}}, \frac{\partial \mathbf{u}_{\mathbf{0}}}{\partial z}, \rho_{0}, \nabla \rho_{0}$ and $\frac{\partial \rho_{0}}{\partial z} \in L^{\infty}(\Omega)$, then the solution $(\mathbf{u}, \rho, P)$ of problem (2.1) satisfies the following estimates:

(i) $\|\mathbf{u}\|_{L^{2}\left(0, T, H^{2}(\Omega)\right)} \leq C\|\mathbf{f}\|_{\mathcal{U}}$,

(ii) $\|\rho\|_{L^{2}\left(0, T, H^{2}(\Omega)\right)} \leq C\|\mathbf{f}\|_{\mathcal{U}}$,

(iii) $\|P\|_{L^{2}\left(0, T, L^{2}\left(\Gamma_{0}\right)\right)} \leq C\|\mathbf{f}\|_{\mathcal{U}}$,

where

$$
\begin{gathered}
\mathcal{U}=\left\{\mathbf{f} \mid \mathbf{f} \in L^{2}\left(0, T, H^{1}\left(\Gamma_{0}\right)\right), \frac{\partial \mathbf{f}}{\partial t} \in L^{2}\left(0, T, L^{2}\left(\Gamma_{0}\right)\right)\right\} \\
\|\mathbf{f}\|_{\mathcal{U}}^{2}=\|\mathbf{f}\|_{L^{2}\left(0, T, H^{1}\left(\Gamma_{0}\right)\right)}^{2}+\left\|\frac{\partial \mathbf{f}}{\partial t}\right\|_{L^{2}\left(0, T, L^{2}\left(\Gamma_{0}\right)\right)}^{2} .
\end{gathered}
$$


Proof. According to the Theorem 2.2 we have:

$$
\begin{aligned}
\|\mathbf{u}\|_{L^{2}\left(0, T, H^{2}(\Omega)\right)}^{2} \leq & C\left(\|\mathbf{u}\|_{L^{2}\left(0, T, \mathcal{V}_{1}\right)}^{2}+\left\|\frac{\partial \mathbf{u}}{\partial t}\right\|_{L^{2}\left(0, T, \mathcal{H}_{1}\right)}^{2}\right. \\
& \left.+\|\rho\|_{L^{2}\left(0, T, \mathcal{H}_{2}\right)}^{2}+\|\mathbf{f}\|_{L^{2}\left(0, T, H^{1}\left(\Gamma_{0}\right)\right)}^{2}\right) \\
\|\rho\|_{L^{2}\left(0, T, H^{2}(\Omega)\right) \leq} & C\left(\|\rho\|_{L^{2}\left(0, T, \mathcal{V}_{2}\right)}^{2}+\left\|\frac{\partial \rho}{\partial t}\right\|_{L^{2}\left(0, T, \mathcal{H}_{2}\right)}^{2}\right. \\
& \left.+\|\mathbf{u}\|_{L^{2}\left(0, T, \mathcal{H}_{1}\right)}^{2}\right), \\
\|P\|_{L^{2}\left(0, T, L^{2}\left(\Gamma_{0}\right)\right) \leq} & C\left(\|\mathbf{u}\|_{L^{2}\left(0, T, \mathcal{V}_{1}\right)}^{2}+\left\|\frac{\partial \mathbf{u}}{\partial t}\right\|_{L^{2}\left(0, T, \mathcal{H}_{1}\right)}^{2}\right. \\
& \left.+\|\rho\|_{L^{2}\left(0, T, \mathcal{H}_{2}\right)}^{2}+\|\mathbf{f}\|_{L^{2}\left(0, T, H^{1}\left(\Gamma_{0}\right)\right)}^{2}\right)
\end{aligned}
$$

Setting $\mathbf{v}=\mathbf{u}$ and $\phi=\rho$ in (2.1) gives:

$$
\begin{aligned}
& \frac{d}{d t}|\mathbf{u}|^{2}+2 a_{1}(\mathbf{u}, \mathbf{u})+l_{1}(\mathbf{u}, \mathbf{u})+2 d(\mathbf{u}, \mathbf{u})+\frac{2 g}{\rho_{\text {moy }}}\left(\rho, \int_{-H}^{z} \operatorname{div}(\mathbf{u}) d z^{\prime}\right) \\
& =2\left(\mathbf{f}, \gamma_{0} \mathbf{u}\right)_{\Gamma_{0}} \\
& \frac{d}{d t}|\rho|^{2}+2 a_{2}(\rho, \rho)+l_{2}(\mathbf{u}, \rho, \rho)=0
\end{aligned}
$$

According to the coercivity of $a_{1}$ and $a_{2}$, the result of Proposition 2.1, and by applying Hölder's inequality, we deduce that there exists positive constants $\left(\varepsilon_{i}\right)_{i=1, \ldots, 3}$ as small as wanted such that:

$$
\begin{aligned}
& \frac{d}{d t}|\mathbf{u}|^{2}+\left(2 \alpha_{1}-\varepsilon_{1}\right)\|\mathbf{u}\|_{W}^{2} \leq c_{1}|\mathbf{u}|^{2}+c_{2}|\rho|^{2}+c_{3}|\mathbf{f}|_{\Gamma_{0}}^{2} \\
& \frac{d}{d t}|\rho|^{2}+\left(2 \alpha_{2}-\varepsilon_{2}\right)\|\rho\|^{2} \leq c_{4}|\rho|^{2}+\varepsilon_{3}\|\mathbf{u}\|_{W}^{2} .
\end{aligned}
$$

We choose $\left(\varepsilon_{i}\right)_{i=1, \ldots, 3}$ in order to have $2 \alpha_{1}-\varepsilon_{1}-\varepsilon_{3}>0$ and $2 \alpha_{2}-\varepsilon_{2}>0$. By setting $K=\min \left(2 \alpha_{1}-\varepsilon_{1}-\varepsilon_{3}, 2 \alpha_{2}-\varepsilon_{2}\right)$ and $X=(\mathbf{u}, \rho)$, we so obtain:

$$
\frac{d}{d t}|X|^{2}+K\|X\|_{W}^{2} \leq c_{5}|X|^{2}+c_{6}|\mathbf{f}|_{\Gamma_{0}}^{2},
$$

where, $|X|^{2}=|\mathbf{u}|^{2}+|\rho|^{2}$ and $\|X\|_{W}^{2}=\|\mathbf{u}\|_{W}^{2}+\|\rho\|^{2}$.

The Gronwall lemma now gives

$$
|X|(t)^{2} \leq C\|\mathbf{f}\|_{L^{2}\left(0, T, L^{2}\left(\Gamma_{0}\right)\right)}^{2}, \quad \forall t \in[0, T] .
$$

We deduce from (2.14), (2.15) that

$$
\begin{gathered}
\|X\|_{L^{2}\left(0, T, \mathcal{H}_{1} \times \mathcal{H}_{2}\right)}^{2} \leq C\|\mathbf{f}\|_{L^{2}\left(0, T, L^{2}\left(\Gamma_{0}\right)\right)}^{2}, \\
\|X\|_{L^{2}\left(0, T, \mathcal{V}_{1} \times \mathcal{V}_{2}\right)}^{2} \leq C\|\mathbf{f}\|_{L^{2}\left(0, T, L^{2}\left(\Gamma_{0}\right)\right)}^{2} .
\end{gathered}
$$


Setting $(\mathbf{v}, \phi)=\left(\frac{\partial \mathbf{u}}{\partial t}, \frac{\partial \rho}{\partial t}\right)=\left(\mathbf{u}^{\prime}, \phi^{\prime}\right)$ in $(2.1)$ we obtain

$$
\begin{aligned}
& \left|\mathbf{u}^{\prime}\right|^{2}+a_{1}\left(\mathbf{u}, \mathbf{u}^{\prime}\right)+l_{1}\left(\mathbf{u}, \mathbf{u}^{\prime}\right)+d\left(\mathbf{u}, \mathbf{u}^{\prime}\right)+\frac{g}{\rho_{\text {moy }}}\left(\rho, \int_{-H}^{z} \operatorname{div}\left(\mathbf{u}^{\prime}\right) d z^{\prime}\right) \\
& =\left(\mathbf{f}, \gamma_{0} \mathbf{u}^{\prime}\right)_{\Gamma_{0}} \\
& \left|\rho^{\prime}\right|^{2}+a_{2}\left(\rho, \rho^{\prime}\right)+l_{2}\left(\mathbf{u}, \rho, \rho^{\prime}\right)=0
\end{aligned}
$$

and after integration with respect to time

$$
\begin{aligned}
& 2 \int_{0}^{t}\left|\mathbf{u}^{\prime}\right|^{2}(s) d s+a_{1}(\mathbf{u}(t), \mathbf{u}(t))=-2 \int_{0}^{t} l_{1}\left(\mathbf{u}, \mathbf{u}^{\prime}\right)(s) d s \\
& \quad-\int_{0}^{t} \frac{2 g}{\rho_{\text {moy }}}\left(\rho, \int_{-H}^{z} \operatorname{div}(\mathbf{u}) d z^{\prime}\right)(s) d s-2 \int_{0}^{t} d(\mathbf{u}, \mathbf{u})(s) d s+2\left(\mathbf{f}(t), \gamma_{0} \mathbf{u}(t)\right)_{\Gamma_{0}} \\
& \quad-2 \int_{0}^{t}\left(\mathbf{f}^{\prime}(s), \gamma_{0} \mathbf{u}(s)\right)_{\Gamma_{0}} d s \\
& 2 \int_{0}^{t}\left|\rho^{\prime}\right|^{2}(s) d s+a_{2}(\rho(t), \rho(t))=-2 \int_{0}^{t} l_{2}\left(\mathbf{u}, \rho, \rho^{\prime}\right)(s) d s, \forall t \in[0, T] .
\end{aligned}
$$

Since

$$
\begin{aligned}
& l_{1}\left(\mathbf{u}, \mathbf{u}^{\prime}\right)=b_{1}\left(\mathbf{u}, \mathbf{u}_{\mathbf{0}}, \mathbf{u}^{\prime}\right)+b_{1}\left(\mathbf{u}_{\mathbf{0}}, \mathbf{u}, \mathbf{u}^{\prime}\right), \\
& l_{2}\left(\mathbf{u}, \rho, \rho^{\prime}\right)=b_{2}\left(\mathbf{u}, \rho_{0}, \rho^{\prime}\right)+b_{2}\left(\mathbf{u}_{\mathbf{0}}, \rho, \rho^{\prime}\right),
\end{aligned}
$$

which yields, according to the result of Proposition 2.1,

$$
l_{1}\left(\mathbf{u}, \mathbf{u}^{\prime}\right)+d\left(\mathbf{u}, \mathbf{u}^{\prime}\right)+\frac{g}{\rho_{\text {moy }}}\left(\rho, \int_{-H}^{z} \operatorname{div}\left(\mathbf{u}^{\prime}\right) d z^{\prime}\right) \leq C_{1}(\|\mathbf{u}\|+|\rho|)\left|\mathbf{u}^{\prime}\right|
$$

and

$$
l_{2}\left(\mathbf{u}, \rho, \rho^{\prime}\right) \leq C_{2}(\|\rho\|+|\mathbf{u}|)\left|\rho^{\prime}\right| .
$$

Applying the coercivity of $a_{1}$ and $a_{2}$ yields

$$
\begin{aligned}
& 2 \int_{0}^{t}\left|\mathbf{u}^{\prime}\right|^{2}(s) d s+\alpha_{1}\|\mathbf{u}\|^{2} \\
& \leq C_{1} \int_{0}^{t}(\|\mathbf{u}\|+|\rho|)\left|\mathbf{u}^{\prime}\right| d s+C_{3} \int_{0}^{t}\|\mathbf{u}\|\left(\left|\mathbf{f}^{\prime}\right|_{\Gamma_{0}}+|\mathbf{f}|_{\Gamma_{0}}\right) d s, \\
& 2 \int_{0}^{t}\left|\rho^{\prime}\right|^{2}(s) d s+\alpha_{2}\|\rho\|^{2} \leq C_{2} \int_{0}^{t}(\|\rho\|+|\mathbf{u}|)\left|\rho^{\prime}\right| d s .
\end{aligned}
$$

Since $\mathbf{f}(t)=\int_{0}^{t} \mathbf{f}^{\prime}(s) d s$, we have

$$
|\mathbf{f}|_{\Gamma_{0}}^{2}(t) \leq C\left\|\mathbf{f}^{\prime}\right\|_{L^{2}\left(0, T, L^{2}\left(\Gamma_{0}\right)\right)}^{2}, \forall t \in[0, T],
$$


and according to (2.16) we obtain

$$
\begin{gathered}
\left\|\mathbf{u}^{\prime}\right\|_{L^{2}\left(0, T, \mathcal{H}_{1}\right)}^{2} \leq C\|\mathbf{f}\|_{\mathcal{U}}^{2}, \\
\left\|\rho^{\prime}\right\|_{L^{2}\left(0, T, \mathcal{H}_{2}\right)}^{2} \leq C\|\mathbf{f}\|_{\mathcal{U}}^{2} .
\end{gathered}
$$

We deduce from (2.13), (2.16) and (2.17) the estimates (i)-(iii).

\subsection{The optimal control problem.}

The problem is controlled by the variability of the wind-stress $\mathbf{f}$. The observation is the surface pressure, deduced from altimetric measurements. Controls and observations are thus defined on $\Gamma_{0}$. Thereby

$$
\mathcal{U}=\left\{\mathbf{f} \mid \mathbf{f} \in L^{2}\left(0, T, H^{1}\left(\Gamma_{0}\right)\right), \frac{\partial \mathbf{f}}{\partial t} \in L^{2}\left(0, T, L^{2}\left(\Gamma_{0}\right)\right)\right\}
$$

will be the control space; $\mathcal{B}=L^{2}\left(0, T, L^{2}\left(\Gamma_{0}\right)\right)$ will be the observation space. For each control $\mathbf{f},(\mathbf{u}(\mathbf{f}), \rho(\mathbf{f}), P(\mathbf{f}))$ is the solution of the weak problem (2.2), and the cost function $\mathcal{J}$ is defined by

$$
\mathcal{J}(\mathbf{f})=\frac{1}{2}\left\|P(\mathbf{f})-P_{o b s}\right\|_{\mathcal{B}}^{2}+\frac{\alpha}{2}\|\mathbf{f}\|_{\mathcal{U}}^{2},
$$

$P_{\text {obs }} \in \mathcal{B}$ is the observation. $\alpha$ is a given positive constant $(\alpha \neq 0)$. The optimal control problem then is as follows:

$$
\begin{array}{r}
\text { Find } \mathbf{f} \in \mathcal{U} \text { such that } \\
\mathcal{J}(\mathbf{f})=\inf _{\mathbf{g} \in \mathcal{U}} \mathcal{J}(\mathbf{g}) .
\end{array}
$$

Remark 2.5. $P$ is defined regardless of any time-dependant function. We now determine this function by setting the condition

$$
\int_{\Gamma_{0}} P d \Gamma=\int_{\Gamma_{0}} P_{o b s} d \Gamma
$$

Proposition 2.5. Problem (2.19) admits one unique solution $\mathbf{f} \in \mathcal{U}$.

Proof. For $(\mathbf{f}, \mathbf{g}) \in \mathcal{U}^{2}$, we set

$$
\mathcal{D}(\mathbf{f}, \mathbf{g})=(P(\mathbf{f}), P(\mathbf{g}))_{\mathcal{B}}+\alpha(\mathbf{f}, \mathbf{g})_{\mathcal{U}}
$$

and

$$
\mathcal{S}(\mathbf{f})=\left(P(\mathbf{f}), P_{o b s}\right)_{\mathcal{B}}
$$

We have then,

$$
\mathcal{J}(\mathbf{f})=\frac{1}{2} \mathcal{D}(\mathbf{f}, \mathbf{f})-\mathcal{S}(\mathbf{f})+\frac{1}{2}\left\|P_{o b s}\right\|_{\mathcal{B}}^{2}
$$


By using the Proposition 2.4, we prove easily that $\mathcal{J}$ is continuous, coercive and strictly convex on $\mathcal{U}$. Therefore, according to [13], [17] we deduce the existence and uniqueness of the solution of problem (2.19).

$\mathbf{f} \in \mathcal{U}$ is solution of problem (2.19) if and only if $\mathcal{J}^{\prime}(\mathbf{f})=0$. In order to characterize the optimal control $\mathbf{f}$ we introduce the adjoint problem associated with problem (2.2).

For a wind-stress $\mathbf{f} \in \mathcal{U},(\mathbf{u}(\mathbf{f}), \rho(\mathbf{f}), P(\mathbf{f}))$ is the solution of (2.2). We note $\left(\mathbf{u}^{*}(\mathbf{f}), \rho^{*}(\mathbf{f}), P^{*}(\mathbf{f})\right)$ the adjoint state which is the solution of the adjoint problem:

$$
\begin{aligned}
& \left(-\frac{\partial \mathbf{u}^{*}(\mathbf{f})}{\partial t}, \mathbf{v}\right)+a_{1}\left(\mathbf{u}^{*}(\mathbf{f}), \mathbf{v}\right)+l_{1}\left(\mathbf{v}, \mathbf{u}^{*}(\mathbf{f})\right)+\left(\rho^{*}(\mathbf{f}) \nabla \rho_{0}, \mathbf{v}\right) \\
& \quad-\left(\rho^{*}(\mathbf{f}) \frac{\partial \rho_{0}}{\partial z}, \int_{-H}^{z} \operatorname{div}(\mathbf{v}) d z^{\prime}\right)+\frac{1}{\rho_{\text {moy }}}\left(P^{*}(\mathbf{f}), \int_{-H}^{0} \operatorname{div}(\mathbf{v}) d z^{\prime}\right)_{\Gamma_{0}} \\
& \quad-\left(\nu_{1 v} \int_{-H}^{0} \operatorname{div}\left(\mathbf{u}^{*}(\mathbf{f})\right) d z^{\prime}, \gamma_{0} \operatorname{div}(\mathbf{v})\right)_{\Gamma_{0}}-d\left(\mathbf{u}^{*}(\mathbf{f}), \mathbf{v}\right)=0, \\
& \left(-\frac{\partial \rho^{*}(\mathbf{f})}{\partial t}, \phi\right)+a_{2}\left(\rho^{*}(\mathbf{f}), \phi\right)+b_{2}\left(\mathbf{u}_{\mathbf{0}}, \phi, \rho^{*}(\mathbf{f})\right) \\
& \quad-\frac{g}{\rho_{m o y}}\left(\int_{-H}^{z} \operatorname{div}\left(\mathbf{u}^{*}(\mathbf{f})\right) d z^{\prime}, \phi\right)=0, \\
& \frac{1}{\rho_{m o y}}\left(\int_{-H}^{0} \operatorname{div}\left(\mathbf{u}^{*}(\mathbf{f})\right) d z^{\prime}, Q\right)_{\Gamma_{0}}=\left(P(\mathbf{f})-P_{o b s}, Q\right)_{\Gamma_{0}}, \\
& \forall(\mathbf{v}, \phi, Q) \in\left(\left(\mathcal{W}_{1} \times \mathcal{V}_{2}\right) \cap H^{2}\right) \times L^{2}\left(\Gamma_{0}\right), \\
& \mathbf{u}^{*}(\mathbf{f})(T)=\mathbf{0}, \rho^{*}(\mathbf{f})(T)=0 \\
& \int_{\Gamma_{0}}\left(P(\mathbf{f})-P_{o b s}\right) d \Gamma_{0}=0 .
\end{aligned}
$$

Proposition 2.6. The adjoint problem (2.20) admits one unique solution such that:

$$
\left(\mathbf{u}^{*}(\mathbf{f}), \rho^{*}(\mathbf{f}), P^{*}(\mathbf{f})\right) \in L^{2}\left(0, T, \mathcal{W}_{1} \times \mathcal{V}_{2} \times L^{2}\left(\Gamma_{0}\right)\right)
$$

Proof. The compatibility condition $\int_{\Gamma_{0}}\left(P(\mathbf{f})-P_{o b s}\right) d \Gamma_{0}=0$ is satisfied. So, almost everywhere in $] 0, T\left[\right.$, there exists $\mathbf{u}_{1} \in \mathcal{W}_{1}$ such that:

$$
\frac{1}{\rho_{\text {moy }}}\left(\int_{-H}^{0} \operatorname{div}\left(\mathbf{u}_{1}\right) d z^{\prime}, Q\right)_{\Gamma_{0}}=\left(P(\mathbf{f})-P_{o b s}, Q\right)_{\Gamma_{0}}, \forall Q \in L^{2}\left(\Gamma_{0}\right) .
$$


Set $\tilde{\mathbf{u}}(\mathbf{f})=\mathbf{u}^{*}(\mathbf{f})-\mathbf{u}_{1}$, equations (2.20) can be written:

$$
\begin{aligned}
& \left(-\frac{\partial \tilde{\mathbf{u}}(\mathbf{f})}{\partial t}, \mathbf{v}\right)+a_{1}(\tilde{\mathbf{u}}(\mathbf{f}), \mathbf{v})+l_{1}(\mathbf{v}, \tilde{\mathbf{u}}(\mathbf{f}))+\left(\rho^{*}(\mathbf{f}) \nabla \rho_{0}, \mathbf{v}\right) \\
& \quad-\left(\rho^{*}(\mathbf{f}) \frac{\partial \rho_{0}}{\partial z}, \int_{-H}^{z} \operatorname{div}(\mathbf{v}) d z^{\prime}\right)+\frac{1}{\rho_{m o y}}\left(P^{*}(\mathbf{f}), \int_{-H}^{0} \operatorname{div}(\mathbf{v}) d z^{\prime}\right)_{\Gamma_{0}} \\
& \quad-d(\tilde{\mathbf{u}}(\mathbf{f}), \mathbf{v})=(G, \mathbf{v}), \\
& \left(-\frac{\partial \rho^{*}(\mathbf{f})}{\partial t}, \phi\right)+a_{2}\left(\rho^{*}(\mathbf{f}), \phi\right)+b_{2}\left(\mathbf{u}_{\mathbf{0}}, \phi, \rho^{*}(\mathbf{f})\right) \\
& \quad-\frac{g}{\rho_{m o y}}\left(\int_{-H}^{z} \operatorname{div}\left(\tilde{\mathbf{u}}(\mathbf{f}) d z^{\prime}, \phi\right)=(\Psi, \phi),\right. \\
& \left(-\int_{-H}^{0} \operatorname{div}\left(\tilde{\mathbf{u}}(\mathbf{f}) d z^{\prime}, Q\right)_{\Gamma_{0}}=0, \forall(\mathbf{v}, \phi, Q) \in \mathcal{W}_{1} \times \mathcal{V}_{2} \times L^{2}\left(\Gamma_{0}\right),\right. \\
& \tilde{\mathbf{u}}(\mathbf{f})(T)=\mathbf{0}, \rho^{*}(\mathbf{f})(T)=0, \\
& \int_{\Gamma_{0}}\left(P(\mathbf{f})-P_{o b s}\right) d \Gamma_{0}=0 .
\end{aligned}
$$

Problem (2.21), being similar to problem 2.2, admits one unique solution such that

$$
\left(\tilde{\mathbf{u}}(\mathbf{f}), \rho^{*}(\mathbf{f}), P^{*}(\mathbf{f})\right) \in L^{2}\left(0, T, \mathcal{V}_{1} \times \mathcal{V}_{2} \times L^{2}\left(\Gamma_{0}\right)\right)
$$

We can deduce that there exists

$$
\left(\mathbf{u}^{*}(\mathbf{f}), \rho^{*}(\mathbf{f}), P^{*}(\mathbf{f})\right) \in L^{2}\left(0, T, \mathcal{W}_{1} \times \mathcal{V}_{2} \times L^{2}\left(\Gamma_{0}\right)\right)
$$

and verifies (2.20). The existence of a solution being proved, demonstrating the uniqueness is fairly simple.

Proposition 2.7. $\mathcal{J}^{\prime}(\mathbf{f})=0$ if and only if $\alpha \mathbf{f}-\Lambda^{-1} \gamma_{0} \mathbf{u}^{*}(\mathbf{f})=0$ in $\mathcal{U}$. $\Lambda$ is the canonical isomorphism $\mathcal{U} \longrightarrow \mathcal{U}^{\prime}$ such that

$$
\langle\mathbf{g}, \mathbf{v}\rangle_{\mathcal{U}, \mathcal{U}^{\prime}}=(\Lambda \mathbf{g}, \mathbf{v})_{\mathcal{U}^{\prime}}=\left(\mathbf{g}, \Lambda^{-1} \mathbf{v}\right)_{\mathcal{U}}, \forall(\mathbf{g}, \mathbf{v}) \in \mathcal{U} \times \mathcal{U}^{\prime}
$$

Proof. $\left(\mathcal{J}^{\prime}(\mathbf{f}), \mathbf{g}\right)=\mathcal{D}(\mathbf{f}, \mathbf{g})-S(\mathbf{g})=\left(P(\mathbf{f})-P_{o b s}, P(\mathbf{g})\right)_{\mathcal{B}}+\alpha(\mathbf{f}, \mathbf{g})_{\mathcal{U}}$, $\forall(\mathbf{f}, \mathbf{g}) \in \mathcal{U}^{2}$.

The third part of equation (2.20) implies

$$
\begin{aligned}
& \left(\mathcal{J}^{\prime}(\mathbf{f}), \mathbf{g}\right)=\alpha(\mathbf{f}, \mathbf{g})_{\mathcal{U}}+\frac{1}{\rho_{\text {moy }}}\left(\int_{-H}^{0} \operatorname{div}\left(\mathbf{u}^{*}(\mathbf{f})\right) d z^{\prime}, P(\mathbf{g})\right)_{\mathcal{B}} \\
& \forall(\mathbf{f}, \mathbf{g}) \in \mathcal{U}^{2}
\end{aligned}
$$


Since $(\mathbf{u}(\mathbf{g}), \rho(\mathbf{g}), P(\mathbf{g}))$ is the solution of problem (2.2), we have

$$
\begin{aligned}
& \left(\frac{\partial \mathbf{u}(\mathbf{g})}{\partial t}, \mathbf{v}\right)+a_{1}(\mathbf{u}(\mathbf{g}), \mathbf{v})+l_{1}(\mathbf{u}(\mathbf{g}), \mathbf{v})+d(\mathbf{u}(\mathbf{g}), \mathbf{v}) \\
& \quad+\frac{g}{\rho_{\text {moy }}}\left(\rho(\mathbf{g}), \int_{-H}^{z} \operatorname{div}(\mathbf{v}) d z^{\prime}\right)-\left(\nu_{1 v} \gamma_{0}(\operatorname{div}(\mathbf{u}(\mathbf{g}))), \int_{-H}^{0} \operatorname{div}(\mathbf{v}) d z^{\prime}\right)_{\Gamma_{0}} \\
& \quad+\frac{1}{\rho_{\text {moy }}}\left(\int_{-H}^{0} \operatorname{div}(\mathbf{v}) d z^{\prime}, P(\mathbf{g})\right)_{\Gamma_{0}}=\left(\mathbf{g}, \gamma_{0} \mathbf{v}\right)_{\Gamma_{0}}, \\
& \quad\left(\frac{\partial \rho(\mathbf{g})}{\partial t}, \phi\right)+a_{2}(\rho(\mathbf{g}), \phi)+l_{2}(\mathbf{u}(\mathbf{g}), \rho(\mathbf{g}), \phi)=0, \\
& \quad\left(\int_{-H}^{0} \operatorname{div}(\mathbf{u}(\mathbf{g})) d z^{\prime}, Q\right)_{\Gamma_{0}}=0, \forall(\mathbf{v}, \phi, Q) \in \mathcal{W}_{1} \times \mathcal{V}_{2} \times L^{2}\left(\Gamma_{0}\right) \\
& \quad \mathbf{u}(\mathbf{g})(0)=\mathbf{0}, \rho(\mathbf{g})(0)=0, \\
& \text { and } \operatorname{so}, \operatorname{setting}(\mathbf{v}, \phi, Q)=\left(\mathbf{u}^{*}(\mathbf{f}), \rho^{*}(\mathbf{f}), P^{*}(\mathbf{f})\right) \\
& \left(\frac{\partial \mathbf{u}(\mathbf{g})}{\partial t}, \mathbf{u}^{*}(\mathbf{f})\right)+a_{1}\left(\mathbf{u}(\mathbf{g}), \mathbf{u}^{*}(\mathbf{f})\right)+l_{1}\left(\mathbf{u}(\mathbf{g}), \mathbf{u}^{*}(\mathbf{f})\right) \\
& \quad-\left(\nu_{1 v} \gamma_{0}(\operatorname{div}(\mathbf{u}(\mathbf{g}))), \int_{-H}^{0} \operatorname{div}\left(\mathbf{u}^{*}(\mathbf{f})\right) d z^{\prime}\right)_{\Gamma_{0}}+d\left(\mathbf{u}(\mathbf{g}), \mathbf{u}^{*}(\mathbf{f})\right) \\
& \quad+\frac{g}{\rho_{m o y}}\left(\rho(\mathbf{g}), \int_{-H}^{z} \operatorname{div}\left(\mathbf{u}^{*}(\mathbf{f})\right) d z^{\prime}\right)+\frac{1}{\rho_{m o y}}\left(\int_{-H}^{0} \operatorname{div}\left(\mathbf{u}^{*}(\mathbf{f})\right) d z^{\prime}, P(\mathbf{g})\right)_{\Gamma_{0}} \\
& =\left(\mathbf{g}, \gamma_{0} \mathbf{u}^{*}(\mathbf{f})\right)_{\Gamma_{0}}, \\
& \left(\frac{\partial \rho(\mathbf{g})}{\partial t}, \rho^{*}(\mathbf{f})\right)+a_{2}\left(\rho(\mathbf{g}), \rho^{*}(\mathbf{f})\right)+l_{2}\left(\mathbf{u}(\mathbf{g}), \rho(\mathbf{g}), \rho^{*}(\mathbf{f})\right)=0 \\
& \quad\left(\int_{-H}^{0} \operatorname{div}(\mathbf{u}(\mathbf{g})) d z^{\prime}, P^{*}(\mathbf{f})\right)_{\Gamma_{0}}=0, \\
& \mathbf{u}(\mathbf{g})(0)=\mathbf{0}, \rho(\mathbf{g})(0)=0 .
\end{aligned}
$$

Since $\left(\mathbf{u}^{*}(\mathbf{f}), \rho^{*}(\mathbf{f}), P^{*}(\mathbf{f})\right)$ is the solution of problem $(2.20)$, we thus obtain after integrating by time:

$$
\int_{0}^{T} b_{2}\left(\mathbf{u}(\mathbf{g}), \rho_{0}, \rho^{*}(\mathbf{f})\right) d t=\int_{0}^{T} \frac{g}{\rho_{\text {moy }}}\left(\rho(\mathbf{g}), \int_{-H}^{z} \operatorname{div}\left(\mathbf{u}^{*}(\mathbf{f})\right) d z^{\prime}\right) d t
$$

and

$$
\int_{0}^{T}\left(P(\mathbf{f})-P_{o b s}, P(\mathbf{g})\right)_{\Gamma_{0}} d t=\int_{0}^{T}\left(\mathbf{g}, \gamma_{0} \mathbf{u}^{*}(\mathbf{f})\right)_{\Gamma_{0}} d t .
$$

We can now conclude that $\mathcal{J}^{\prime}(\mathbf{f})=0$ if and only if $\alpha \mathbf{f}-\Lambda^{-1} \gamma_{0} \mathbf{u}^{*}(\mathbf{f})=0$ in $\mathcal{U}$ (according to (2.22)). 
We have proved that the optimal control $\mathbf{f}$, the solution of problem (2.19), is characterized by the following set of equations:

$$
\begin{aligned}
& \left(\frac{\partial \mathbf{u}(\mathbf{f})}{\partial t}, \mathbf{v}\right)+a_{1}(\mathbf{u}(\mathbf{f}), \mathbf{v})+l_{1}(\mathbf{u}(\mathbf{f}), \mathbf{v})+d(\mathbf{u}(\mathbf{f}), \mathbf{v}) \\
& +\frac{g}{\rho_{\text {moy }}}\left(\rho(\mathbf{f}), \int_{-H}^{z} \operatorname{div}(\mathbf{v}) d z^{\prime}\right)-\left(\nu_{1 v} \gamma_{0}(\operatorname{div}(\mathbf{u}(\mathbf{f}))), \int_{-H}^{0} \operatorname{div}(\mathbf{v}) d z^{\prime}\right)_{\Gamma_{0}} \\
& +\frac{1}{\rho_{\text {moy }}}\left(\int_{-H}^{0} \operatorname{div}(\mathbf{v}) d z^{\prime}, P(\mathbf{f})\right)_{\Gamma_{0}}=\left(\mathbf{f}, \gamma_{0} \mathbf{v}\right)_{\Gamma_{0}}, \\
& \left(\frac{\partial \rho(\mathbf{f})}{\partial t}, \phi\right)+a_{2}(\rho(\mathbf{f}), \phi)+l_{2}(\mathbf{u}(\mathbf{f}), \rho(\mathbf{f}), \phi)=0, \\
& \left(\int_{-H}^{0} \operatorname{div}(\mathbf{u}(\mathbf{f})) d z^{\prime}, Q\right)_{\Gamma_{0}}=0 \\
& \left(-\frac{\partial \mathbf{u}^{*}(\mathbf{f})}{\partial t}, \mathbf{v}\right)+a_{1}\left(\mathbf{u}^{*}(\mathbf{f}), \mathbf{v}\right)+l_{1}\left(\mathbf{v}, \mathbf{u}^{*}(\mathbf{f})\right)+\left(\rho^{*}(\mathbf{f}) \nabla \rho_{0}, \mathbf{v}\right) \\
& -\left(\rho^{*}(\mathbf{f}) \frac{\partial \rho_{0}}{\partial z}, \int_{-H}^{z} \operatorname{div}(\mathbf{v}) d z^{\prime}\right)+\frac{1}{\rho_{\text {moy }}}\left(P^{*}(\mathbf{f}), \int_{-H}^{0} \operatorname{div}(\mathbf{v}) d z^{\prime}\right)_{\Gamma_{0}} \\
& -\left(\nu_{1 v} \int_{-H}^{0} \operatorname{div}\left(\mathbf{u}^{*}(\mathbf{f})\right) d z^{\prime}, \gamma_{0} \operatorname{div}(\mathbf{v})\right)_{\Gamma_{0}}-d\left(\mathbf{u}^{*}(\mathbf{f}), \mathbf{v}\right)=0, \\
& \left(-\frac{\partial \rho^{*}(\mathbf{f})}{\partial t}, \phi\right)+a_{2}\left(\rho^{*}(\mathbf{f}), \phi\right)+b_{2}\left(\mathbf{u}_{\mathbf{0}}, \phi, \rho^{*}(\mathbf{f})\right) \\
& -\frac{g}{\rho_{\text {moy }}}\left(\int_{-H}^{z} \operatorname{div}\left(\mathbf{u}^{*}(\mathbf{f})\right) d z^{\prime}, \phi\right)=0, \\
& \frac{1}{\rho_{\text {moy }}}\left(\int_{-H}^{0} \operatorname{div}\left(\mathbf{u}^{*}(\mathbf{f})\right) d z^{\prime}, Q\right)_{\Gamma_{0}}=\left(P(\mathbf{f})-P_{o b s}, Q\right)_{\Gamma_{0}}, \\
& \forall(\mathbf{v}, \phi, Q) \in\left(\left(\mathcal{W}_{1} \times \mathcal{V}_{2}\right) \cap H^{2}\right) \times L^{2}\left(\Gamma_{0}\right), \\
& \int_{\Gamma_{0}}\left(P(\mathbf{f})-P_{o b s}\right) d \Gamma_{0}=0 \\
& \mathbf{u}^{*}(\mathbf{f})(T)=\mathbf{u}(\mathbf{f})(0)=\mathbf{0}, \rho^{*}(\mathbf{f})(T)=\rho(\mathbf{f})(0)=0, \\
& \alpha \mathbf{f}-\Lambda^{-1} \gamma_{0} \mathbf{u}^{*}(\mathbf{f})=0 \text { in } \mathcal{U} .
\end{aligned}
$$




\section{The two dimensional cyclic problem}

\subsection{Variational formulation.}

In order to study problem (1.10), we introduce the following functional spaces:

$$
\begin{aligned}
& \mathcal{V}_{0}=\left\{\left(\mathbf{v}, v_{3}\right) \in\left(H^{1}(\omega)\right)^{3} \mid \operatorname{div}_{0}(\mathbf{v})+\frac{\partial v_{3}}{\partial z}=0,\left(\mathbf{v}, v_{3}\right) \cdot \mathbf{n}_{0}=0 \text { on } \gamma\right\}, \\
& \mathcal{H}_{1 c}=\left\{\mathbf{v} \in\left(L_{c}^{2}(\omega)\right)^{2} \mid(\mathbf{v}, 0) \cdot \mathbf{n}_{0}=0 \text { on } \gamma_{0} \cup \gamma_{3} \cup \gamma_{4} \cup \gamma_{5}\right\}, \\
& \mathcal{W}_{1 c}=\left\{\mathbf{v} \in\left(H_{c}^{1}(\omega)\right)^{2} \mid W_{m}(\mathbf{v})=-\int_{-H}^{z} \operatorname{div}_{m}(\mathbf{v}) d z^{\prime} \in H_{c}^{1}(\omega),\right. \\
& \left.\mathbf{v}=\mathbf{0} \text { on } \gamma_{5},(\mathbf{v}, 0) \cdot \mathbf{n}_{0}=0 \text { on } \gamma_{0} \cup \gamma_{3} \cup \gamma_{4}\right\}, \\
& \mathcal{V}_{1 c}=\left\{\mathbf{v} \in \mathcal{W}_{1 c} \mid \int_{-H}^{0} \operatorname{div}_{m}(\mathbf{v}) d z=0\right\}, \\
& \mathcal{H}_{2 c}=L_{c}^{2}(\omega), \\
& \mathcal{V}_{2 c}=\left\{\phi \in H_{c}^{1}(\omega) \mid \phi=0 \text { on } \gamma_{5} \cup \gamma_{3} \cup \gamma_{4}\right\},
\end{aligned}
$$

where, $\mathbf{n}_{0}=\left(0, n_{1}, n_{2}\right), \mathbf{n}=\left(n_{1}, n_{2}\right)$ the unit outward vector normal to $\gamma$.

Let $\mathcal{X}$ be a real Hilbert space, $\mathcal{X}_{c}$ denotes the complex space defined by: $\mathbf{v} \in \mathcal{X}_{c}$ if $\mathbf{v}=\mathbf{v}_{1}+i \mathbf{v}_{2}$, with real part $\mathbf{v}_{1} \in \mathcal{X}$ and imaginary part $\mathbf{v}_{2} \in \mathcal{X}$. The norm in $\mathcal{X}_{c}$ is defined by

$$
\|\mathbf{v}\|_{\mathcal{X}_{c}}^{2}=\left\|\mathbf{v}_{1}\right\|_{\mathcal{X}}^{2}+\left\|\mathbf{v}_{2}\right\|_{\mathcal{X}}^{2}
$$

where $|\cdot|_{1, \omega}$ and $\|\cdot\|_{1, \omega}$ denotes the semi-norm, and the norm on $H_{c}^{1}(\omega)$. They are equivalent on $\mathcal{W}_{1 c}, \mathcal{V}_{1 c}$ and $\mathcal{V}_{2 c}$. We set $\|\mathbf{u}\|=|\mathbf{u}|_{1, \omega},\|\phi\|=$ $|\phi|_{1, \omega},|\mathbf{u}|$ and $|\phi|$ denotes the norm in $L_{c}^{2}(\Omega)$.

$\mathcal{W}_{1 c}$ (resp. $\mathcal{V}_{1 c}$ ) is equipped with the following norm:

$$
\|\mathbf{v}\|_{W}^{2}=\|\mathbf{v}\|^{2}+\left\|\int_{-H}^{0} \operatorname{div}_{m}(\mathbf{v}) d z\right\|^{2}, \forall \mathbf{v} \in \mathcal{W}_{1 c}\left(\operatorname{resp} . \mathbf{v} \in \mathcal{V}_{1 c}\right),
$$

and $\mathcal{V}_{2 c}$ is equipped with the norm $\|\phi\|, \forall \phi \in \mathcal{V}_{2 c}$.

\section{Lemma 3.1.}

$$
\begin{aligned}
& \left(\nabla_{m} \mathcal{L}_{m}(\mathbf{u}), \mathbf{v}\right)=\nu_{1 h}\left(\nabla_{m}\left(\int_{-H}^{z} \operatorname{div}_{m}(\mathbf{u}) d z^{\prime}\right), \nabla_{m}\left(\int_{-H}^{z} \operatorname{div}_{m}(\mathbf{v}) d z^{\prime}\right)\right) \\
& \quad+\left(\nu_{1 v} \operatorname{div}_{m}(\mathbf{u}), \operatorname{div}_{m}(\mathbf{v})\right)-\left(\nu_{1 v} \gamma_{0}\left(\operatorname{div}_{m}(\mathbf{u})\right), \int_{-H}^{0} \operatorname{div}_{m}(\mathbf{v}) d z^{\prime}\right)_{\gamma_{0}} \\
& \forall(\mathbf{u}, \mathbf{v}) \in\left(\mathcal{W}_{1 c} \cap H_{c}^{2}\right)^{2} .
\end{aligned}
$$


Proof. Since

$$
\left(\nabla_{m} \mathcal{L}_{m}(\mathbf{u}), \mathbf{v}\right)=\left(\nabla_{m}\left(\int_{z}^{0} L_{m}(\mathbf{u}) d z^{\prime}\right), \mathbf{v}\right),
$$

by using Green's formula we obtain

$$
\left(\nabla_{m} \mathcal{L}_{m}(\mathbf{u}), \mathbf{v}\right)=-\left(\int_{z}^{0} L_{m}(\mathbf{u}) d z^{\prime}, \operatorname{div}_{m}(\mathbf{v})\right)+\int_{\gamma}\left(\int_{z}^{0} L_{m}(\mathbf{u}) d z^{\prime}\right) \overline{(\mathbf{v}, 0)} \cdot \mathbf{n}_{0} d \gamma
$$

where $\mathbf{v}$ is any element in $\mathcal{W}_{1 c}$. We obtain

$$
\left(\nabla_{m} \mathcal{L}_{m}(\mathbf{u}), \mathbf{v}\right)=-\left(\int_{z}^{0} L_{m}(\mathbf{u}) d z^{\prime}, \operatorname{div}_{m}(\mathbf{v})\right),
$$

By integrating by part with respect to $z$ gives

$$
\left(\nabla_{m} \mathcal{L}_{m}(\mathbf{u}), \mathbf{v}\right)=\left(L_{m}(\mathbf{u}), \int_{-H}^{z} \operatorname{div}_{m}(\mathbf{v}) d z^{\prime}\right) .
$$

Since

$$
L_{m}(\mathbf{u})=-\nu_{1 h} \Delta_{m} W_{m}(\mathbf{u})-\frac{\partial}{\partial z}\left(\nu_{1 v} \frac{\partial W_{m}(\mathbf{u})}{\partial z}\right),
$$

by applying Green's formula, we obtain

$$
\begin{aligned}
& \left(\nabla_{m} \mathcal{L}_{m}(\mathbf{u}), \mathbf{v}\right)=\nu_{1 h}\left(\nabla_{m}\left(\int_{-H}^{z} \operatorname{div}_{m}(\mathbf{u}) d z^{\prime}\right), \nabla_{m}\left(\int_{-H}^{z} \operatorname{div}_{m}(\mathbf{v}) d z^{\prime}\right)\right) \\
& \quad+\left(\nu_{1 v} \operatorname{div}_{m}(\mathbf{u}), \operatorname{div}_{m}(\mathbf{v})\right)-\left(\nu_{1 v} \gamma_{0}\left(\operatorname{div}_{m}(\mathbf{u})\right), \int_{-H}^{0} \operatorname{div}_{m}(\mathbf{v}) d z^{\prime}\right)_{\gamma_{0}} .
\end{aligned}
$$

Remark 3.1. If $\mathbf{v} \in \mathcal{V}_{1 c}$, we have

$$
\begin{aligned}
\left(\nabla_{m} \mathcal{L}_{m}(\mathbf{u}), \mathbf{v}\right)= & \nu_{1 h}\left(\nabla_{m}\left(\int_{-H}^{z} \operatorname{div}_{m}(\mathbf{u}) d z^{\prime}\right), \nabla_{m}\left(\int_{-H}^{z} \operatorname{div}_{m}(\mathbf{v}) d z^{\prime}\right)\right) \\
& +\left(\nu_{1 v} \operatorname{div}_{m}(\mathbf{u}), \operatorname{div}_{m}(\mathbf{v})\right)
\end{aligned}
$$

We now define:

$$
\begin{aligned}
& a_{11 m}(\mathbf{u}, \mathbf{v})=\nu_{1 h}\left(\nabla_{m} \mathbf{u}, \nabla_{m} \mathbf{v}\right)+\left(\nu_{1 v} \frac{\partial \mathbf{u}}{\partial z}, \frac{\partial \mathbf{v}}{\partial z}\right) \\
& a_{12 m}(\mathbf{u}, \mathbf{v})=\nu_{1 h}\left(\nabla_{m} W_{m}(\mathbf{u}), \nabla_{m} W_{m}(\mathbf{v})\right)+\left(\nu_{1 v} \operatorname{div}_{m}(\mathbf{u}), \operatorname{div}_{m}(\mathbf{v})\right), \\
& a_{1 m}(\mathbf{u}, \mathbf{v})=a_{11 m}(\mathbf{u}, \mathbf{v})+a_{12 m}(\mathbf{u}, \mathbf{v}) \\
& a_{2 m}(\rho, \phi)=\nu_{2 h}\left(\nabla_{m} \rho, \nabla_{m} \phi\right)+\left(\nu_{2 v} \frac{\partial \rho}{\partial z}, \frac{\partial \phi}{\partial z}\right) \\
& d(\mathbf{u}, \mathbf{v})=\left(\mathbf{F} \wedge_{2} \mathbf{u}, \mathbf{v}\right)
\end{aligned}
$$




$$
\begin{aligned}
& b_{1 m}\left(\mathbf{u}_{\mathbf{0}}, \mathbf{u}, \mathbf{v}\right)=\left(\left(\mathbf{u}_{\mathbf{0}} \nabla_{m}\right) \mathbf{u}, \mathbf{v}\right)+\left(w_{0} \frac{\partial \mathbf{u}}{\partial z}, \mathbf{v}\right) \\
& b_{2 m}\left(\mathbf{u}_{\mathbf{0}}, \rho, \phi\right)=\left(\left(\mathbf{u}_{\mathbf{0}} \nabla_{m}\right) \rho, \phi\right)+\left(w_{0} \frac{\partial \rho}{\partial z}, \phi\right) \\
& c_{1 m}(\mathbf{u}, \mathbf{v}, \mathbf{w})=\left(\left(\mathbf{u} \nabla_{0}\right) \mathbf{v}, \mathbf{w}\right)+\left(W_{m}(\mathbf{u}) \frac{\partial \mathbf{v}}{\partial z}, \mathbf{w}\right), \\
& c_{2 m}(\mathbf{u}, \rho, \phi)=\left(\left(\mathbf{u} \nabla_{0}\right) \rho, \phi\right)+\left(W_{m}(\mathbf{u}) \frac{\partial \rho}{\partial z}, \phi\right), \\
& l_{1 m}(\mathbf{u}, \mathbf{v})=b_{1 m}\left(\mathbf{u}_{\mathbf{0}}, \mathbf{u}, \mathbf{v}\right)+c_{1 m}\left(\mathbf{u}, \mathbf{u}_{\mathbf{0}}, \mathbf{v}\right), \\
& L_{1 m}(\mathbf{u}, \rho, \mathbf{v})=l_{1 m}(\mathbf{u}, \mathbf{v})+d(\mathbf{u}, \mathbf{v})+\frac{g}{\rho_{m o y}}\left(\rho, \int_{-H}^{z} \operatorname{div}_{m}(\mathbf{v}) d z^{\prime}\right), \\
& l_{2 m}(\mathbf{u}, \rho, \phi)=b_{2 m}\left(\mathbf{u}_{\mathbf{0}}, \rho, \phi\right)+c_{2 m}\left(\mathbf{u}, \rho_{0}, \phi\right)
\end{aligned}
$$

where $R e($.$) is the real part of (.).$

Lemma 3.2. Suppose that $\mathbf{f} \in L^{2}\left(0, T, L_{c}^{2}\left(\gamma_{0}\right)\right)$ and that $(\mathbf{u}, \rho, P)$ is a sufficiently regular. Then:

(i) $-\nu_{1 h}\left(\Delta_{m} \mathbf{u}, \mathbf{v}\right)-\left(\frac{\partial}{\partial z}\left(\nu_{1 v} \frac{\partial \mathbf{u}}{\partial z}\right), \mathbf{v}\right)=a_{1 m}(\mathbf{u}, \mathbf{v})-\left(\mathbf{f}, \gamma_{0} \mathbf{v}\right)_{\gamma_{0}}, \forall \mathbf{v} \in \mathcal{W}_{1 c}$,

(ii) $-\nu_{1 h}\left(\Delta_{m} \rho, \phi\right)-\left(\frac{\partial}{\partial z}\left(\nu_{1 v} \frac{\partial \rho}{\partial z}\right), \phi\right)=a_{2 m}(\rho, \phi), \forall \phi \in \mathcal{V}_{2 c}$,

(iii) $\left(\nabla_{m}\left(\int_{z}^{0} \rho d z^{\prime}\right), \mathbf{v}\right)=-\left(\int_{z}^{0} \rho d z^{\prime}, \operatorname{div}_{m}(\mathbf{v})\right)=\left(\rho, \int_{-H}^{z} \operatorname{div}_{m}(\mathbf{v}) d z^{\prime}\right)$,

$\forall \mathbf{v} \in \mathcal{W}_{1 c}$

(iv) $\left(\nabla_{m} P, \mathbf{v}\right)=-\left(P, \int_{-H}^{0} \operatorname{div}_{m}(\mathbf{v}) d z^{\prime}\right)_{\gamma_{0}}, \forall \mathbf{v} \in \mathcal{W}_{1 c}$.

$\gamma_{0} \mathbf{v}$ denotes the trace of $\mathbf{v}$ on $\gamma_{0}$, and $\left(\mathbf{f}, \gamma_{0} \mathbf{v}\right)_{\gamma_{0}}$ denotes the scalar product in $L_{c}^{2}\left(\gamma_{0}\right)$, which makes sense if $\mathbf{v} \in \mathcal{W}_{1 c}$.

Proof. Results (i)-(ii) are deduced from the definition of the spaces $\mathcal{W}_{1 c}, \mathcal{V}_{1 c}$ and $\mathcal{V}_{2 c}$, and from the boundary conditions satisfied by $(\mathbf{u}, \rho)$ on $\gamma$.

$$
\begin{aligned}
& \left(\nabla_{m}\left(\int_{z}^{0} \rho d z^{\prime}\right), \mathbf{v}\right)=\left(i m \int_{z}^{0} \rho d z^{\prime}, v_{1}\right)+\left(\frac{\partial}{\partial y}\left(\int_{z}^{0} \rho d z^{\prime}\right), v_{2}\right) \\
& =-\left(\int_{z}^{0} \rho d z^{\prime}, i m v_{1}\right)-\left(\int_{z}^{0} \rho d z^{\prime}, \frac{\partial v_{2}}{\partial y}\right)+\int_{\gamma}\left(\int_{z}^{0} \rho d z^{\prime}\right) \cdot \overline{(\mathbf{v}, 0)} \cdot \mathbf{n}_{0} d \gamma .
\end{aligned}
$$

Since $\mathbf{v} \in \mathcal{W}_{1 c}$ we have $(\mathbf{v}, 0) . \mathbf{n}_{0}=0$ on $\gamma$ and therefore

$$
\left(\nabla_{m}\left(\int_{z}^{0} \rho d z^{\prime}\right), \mathbf{v}\right)=-\left(\int_{z}^{0} \rho d z^{\prime}, \operatorname{div}_{m}(\mathbf{v})\right), \forall \mathbf{v} \in \mathcal{W}_{1 c} .
$$


By integrating by parts with respect to $z$ gives

$$
-\left(\int_{z}^{0} \rho d z^{\prime}, \operatorname{div}_{m}(\mathbf{v})\right)=\left(\rho, \int_{-H}^{z} \operatorname{div}_{m}(\mathbf{v}) d z^{\prime}\right) .
$$

(iv) According to Green's formula and boundary conditions verified by $\mathbf{v}$, we then have: $\left(\nabla_{m} P, \mathbf{v}\right)=-\left(P, \operatorname{div}_{m}(\mathbf{v})\right)$.

Since $P$ is only a function of $y$ we can deduce that

$$
\left(\nabla_{m} P, \mathbf{v}\right)=-\left(P, \int_{-H}^{0} \operatorname{div}_{m}(\mathbf{v}) d z^{\prime}\right)_{\gamma_{0}} .
$$

We can now write the two weak formulations of problem (1.10), (1.11), (1.6) satisfied by $(\mathbf{u}, \rho, P)$ :

Find $(\mathbf{u}, \rho) \in L^{2}\left(0, T, \mathcal{V}_{1 c}\right) \times L^{2}\left(0, T, \mathcal{V}_{2 c}\right)$ such that :

$$
\begin{aligned}
& \left(\frac{\partial \mathbf{u}}{\partial t}, \mathbf{v}\right)+a_{1 m}(\mathbf{u}, \mathbf{v})+L_{1 m}(\mathbf{u}, \rho, \mathbf{v})=\left(\mathbf{f}, \gamma_{0} \mathbf{v}\right), \forall \mathbf{v} \in \mathcal{V}_{1 c} \\
& \left(\frac{\partial \rho}{\partial t}, \phi\right)+a_{2 m}(\rho, \phi)+l_{2 m}(\mathbf{u}, \rho, \phi)=0, \forall \phi \in \mathcal{V}_{2 c} \\
& \mathbf{u}(0)=\mathbf{0}, \rho(0)=0
\end{aligned}
$$

and

Find $(\mathbf{u}, \rho, P) \in L^{2}\left(0, T, \mathcal{W}_{1 c} \cap H_{c}^{2}\right) \times L^{2}\left(0, T, \mathcal{V}_{2 c} \cap H_{c}^{2}\right) \times L^{2}\left(0, T, L_{c}^{2}\left(\gamma_{0}\right)\right)$ such that:

$$
\begin{aligned}
& \left(\frac{\partial \mathbf{u}}{\partial t}, \mathbf{v}\right)+a_{1 m}(\mathbf{u}, \mathbf{v})+L_{1 m}(\mathbf{u}, \rho, \mathbf{v})-\left(\nu_{1 v} \gamma_{0}\left(\operatorname{div}_{m}(\mathbf{u})\right), \int_{-H}^{0} \operatorname{div}_{m}(\mathbf{v}) d z^{\prime}\right)_{\gamma_{0}} \\
& \quad-\frac{1}{\rho_{m o y}}\left(P, \int_{-H}^{0} \operatorname{div}_{m}(\mathbf{v}) d z^{\prime}\right)_{\gamma_{0}}=\left(\mathbf{f}, \gamma_{0} \mathbf{v}\right)_{\gamma_{0}} \\
& \left(\frac{\partial \rho}{\partial t}, \phi\right)+a_{2 m}(\rho, \phi)+l_{2 m}(\mathbf{u}, \rho, \phi)=0, \forall(\mathbf{v}, \phi) \in \mathcal{W}_{1 c} \times \mathcal{V}_{2 c} \\
& -\left(\int_{-H}^{0} \operatorname{div}_{m}(\mathbf{u}) d z^{\prime}, Q\right)_{\gamma_{0}}=0, \forall Q \in L^{2}\left(\gamma_{0}\right) \\
& \mathbf{u}(0)=\mathbf{0}, \rho(0)=0
\end{aligned}
$$

In the two following propositions we are dealing with some properties of operators $a_{1 m}, a_{2 m}, l_{1 m}, L_{1 m}, l_{2 m}$ and $d$.

Proposition 3.1. (i) $a_{1 m}$ (resp. $\left.a_{2 m}\right)$ is a bilinear continuous and coercive form on $\mathcal{W}_{1 c}^{2}$ (resp. on $\left.\mathcal{V}_{2 c}^{2}\right)$, 
(ii) $d$ is a bilinear continuous form on $\mathcal{W}_{1 c}^{2}$ and $d(\mathbf{u}, \mathbf{v})=-\bar{d}(\mathbf{v}, \mathbf{u})$ $(\bar{d}$ denotes the complex conjugate of $d)$.

There exists a positive constant $c$, depending on $m$, such that:

(iii) $\left\|W_{m}(\mathbf{v})\right\|_{L_{c}^{2}(\omega)} \leq c\|\mathbf{v}\|, \forall \mathbf{v} \in \mathcal{W}_{1 c}$

(iv) $\left|\frac{g}{\rho_{\text {moy }}}\left(\rho, \int_{-H}^{z} \operatorname{div}_{m}(\mathbf{v}) d z^{\prime}\right)\right| \leq c\|\rho\|_{L_{c}^{2}(\omega)}\|\mathbf{v}\|$, $\leq c\|\mathbf{v}\|_{L_{c}^{2}(\omega)}\|\rho\|, \forall(\mathbf{v}, \rho) \in \mathcal{W}_{1 c} \times \mathcal{V}_{2 c}$.

Proof. (i) $\nu_{1 h}$ and $\nu_{2 h}$ are constant coefficients, $\nu_{1 v}$ and $\nu_{2 v}$ are bounded functions, therefore the continuity and the coercivity of $a_{1 m}$ and $a_{2 m}$ are easily obtained. The constant of coercivity of $a_{1 m}$ (resp. $a_{2 m}$ ) $\alpha_{1 m}$ (resp. $\left.\alpha_{2 m}\right)$ is then given by $\alpha_{1 m}=\min \left(\nu_{1 h}, m^{2} \nu_{1 h}, \min \left(\nu_{1 v}\right)\right)$ (resp. $\alpha_{2 m}=$ $\left.\min \left(\nu_{2 h}, m^{2} \nu_{2 h}, \min \left(\nu_{2 v}\right)\right)\right)$.

(ii) is easily obtained from the definition of $d: d(\mathbf{u}, \mathbf{v})=\left(\mathbf{F} \wedge_{2} \mathbf{u}, \mathbf{v}\right)$.

(iii) $W_{m}(\mathbf{v})=-\int_{-H}^{z} \operatorname{div}_{m}(\mathbf{v}) d z^{\prime}=-i m \int_{-H}^{z} v_{1} d z^{\prime}-\int_{-H}^{z} \frac{\partial v_{2}}{\partial y} d z^{\prime}$, so

$$
\left|W_{m}(\mathbf{v})\right| \leq \int_{-H}^{0}\left|\operatorname{div}_{m}(\mathbf{v})\right| d z^{\prime} \leq m \int_{-H}^{0}\left|v_{1}\right| d z^{\prime}+\int_{-H}^{0}\left|\frac{\partial v_{2}}{\partial y}\right| d z^{\prime},
$$

and therefore:

$$
\begin{aligned}
\left\|W_{m}(\mathbf{v})\right\|_{L_{c}^{2}(\omega)}^{2} & \leq 2 m^{2} \int_{\omega}\left(\int_{-H}^{0}\left|v_{1}\right| d z^{\prime}\right)^{2} d \omega+2 \int_{\omega}\left(\int_{-H}^{0}\left|\frac{\partial v_{2}}{\partial y}\right| d z^{\prime}\right)^{2} d \omega \\
& \leq 2 m^{2} H\|\mathbf{v}\|_{L_{c}^{2}(\omega)}^{2}+2 H\|\mathbf{v}\|^{2}
\end{aligned}
$$

from which we deduce

$$
\begin{gathered}
\left\|W_{m}(\mathbf{v})\right\|_{L_{c}^{2}(\omega)} \leq c\|\mathbf{v}\| \\
\text { (iv) }\left|\frac{g}{\rho_{m o y}}\left(\rho, \int_{-H}^{z} \operatorname{div}_{m}(\mathbf{v}) d z^{\prime}\right)\right| \leq \frac{g}{\rho_{m o y}}\|\rho\|_{L_{c}^{2}(\omega)}\left\|\int_{-H}^{z} \operatorname{div}_{m}(\mathbf{v}) d z^{\prime}\right\|_{L_{c}^{2}(\omega)} \\
=\frac{g}{\rho_{\text {moy }}}\|\rho\|_{L_{c}^{2}(\omega)}\left\|W_{m}(\mathbf{v})\right\|_{L_{c}^{2}(\omega)} .
\end{gathered}
$$

By using (iii) we then obtain

$$
\left|\frac{g}{\rho_{\text {moy }}}\left(\rho, \int_{-H}^{z} \operatorname{div}_{m}(\mathbf{v}) d z^{\prime}\right)\right| \leq c\|\rho\|_{L_{c}^{2}(\omega)}\|\mathbf{v}\|, \forall(\rho, \mathbf{v}) \in \mathcal{W}_{1 c} \times \mathcal{V}_{2 c} .
$$

By applying lemma 3.2, we have

$$
\frac{g}{\rho_{\text {moy }}}\left(\rho, \int_{-H}^{z} \operatorname{div}_{m}(\mathbf{v}) d z^{\prime}\right)=-\frac{g}{\rho_{\text {moy }}}\left(\nabla_{m}\left(\int_{z}^{0} \rho d z^{\prime}\right), \mathbf{v}\right),
$$


and therefore

$$
\begin{aligned}
\frac{g}{\rho_{\text {moy }}}\left|\left(\rho, \int_{-H}^{z} \operatorname{div}_{m}(\mathbf{v}) d z^{\prime}\right)\right| & \leq \frac{g}{\rho_{\text {moy }}}\left\|\nabla_{m}\left(\int_{z}^{0} \rho d z^{\prime}\right)\right\|_{L_{c}^{2}(\omega)}\|\mathbf{v}\|_{L_{c}^{2}(\omega)} \\
& \leq \frac{g H}{\rho_{\text {moy }}}\left\|\nabla_{m} \rho\right\|_{L_{c}^{2}(\omega)}\|\mathbf{v}\|_{L_{c}^{2}(\omega)} .
\end{aligned}
$$

So there exists a positive constant $c$ such that

$$
\frac{g}{\rho_{\text {moy }}}\left|\left(\rho, \int_{-H}^{z} \operatorname{div}_{m}(\mathbf{v}) d z^{\prime}\right)\right| \leq c\|\rho\|\|\mathbf{v}\|_{L_{c}^{2}(\omega)}, \forall(\mathbf{v}, \rho) \in \mathcal{W}_{1 c} \times \mathcal{V}_{2 c}
$$

Proposition 3.2. (i) If the mean circulation $\left(\mathbf{u}_{\mathbf{0}}, w_{0}, \rho_{0}\right)$ is given such that: $\mathbf{u}_{\mathbf{0}}, w_{0}, \rho_{0}, \nabla_{0} \mathbf{u}_{\mathbf{0}}, \frac{\partial \mathbf{u}_{\mathbf{0}}}{\partial z}, \nabla_{0} \rho_{0}$ and $\frac{\partial \rho_{0}}{\partial z} \in L^{\infty}(\omega)$, then there exists a positive constant $C$ such that:

(a1) $\left|b_{1 m}\left(\mathbf{u}_{\mathbf{0}}, \mathbf{u}, \mathbf{v}\right)\right| \leq C\|\mathbf{u}\|\|\mathbf{v}\|_{L_{c}^{2}(\omega)}, \forall(\mathbf{u}, \mathbf{v}) \in \mathcal{W}_{1 c}^{2}$,

(a2) $\left|b_{2 m}\left(\mathbf{u}_{\mathbf{0}}, \rho, \phi\right)\right| \leq C\|\rho\|\|\phi\|_{L_{c}^{2}(\omega)}, \forall(\rho, \phi) \in \mathcal{V}_{2 c}^{2}$,

(b1) $\left|c_{1 m}\left(\mathbf{u}, \mathbf{u}_{\mathbf{0}}, \mathbf{v}\right)\right| \leq C\|\mathbf{u}\|\|\mathbf{v}\|_{L_{c}^{2}(\omega)}, \forall(\mathbf{u}, \mathbf{v}) \in \mathcal{W}_{1 c}^{2}$,

(b2) $\left|c_{2 m}\left(\mathbf{u}, \rho_{0}, \phi\right)\right| \leq C\|\mathbf{u}\|\|\phi\|_{L_{c}^{2}(\omega)}, \forall(\mathbf{u}, \phi) \in \mathcal{W}_{1 c} \times \mathcal{V}_{2 c}$,

(ii) $b_{1 m}\left(\mathbf{u}_{\mathbf{0}}, \mathbf{u}, \mathbf{v}\right)=-\overline{b_{1 m}}\left(\mathbf{u}_{\mathbf{0}}, \mathbf{v}, \mathbf{u}\right), \forall(\mathbf{u}, \mathbf{v}) \in \mathcal{W}_{1 c}^{2}$,

(iii) $b_{2 m}\left(\mathbf{u}_{\mathbf{0}}, \rho, \phi\right)=-\overline{b_{2 m}}\left(\mathbf{u}_{\mathbf{0}}, \phi, \rho\right), \forall(\rho, \phi) \in \mathcal{V}_{2 c}^{2}$

(iv) $c_{1 m}\left(\mathbf{u}, \mathbf{u}_{\mathbf{0}}, \mathbf{v}\right)=-c_{1 m}\left(\mathbf{u}, \overline{\mathbf{v}}, \mathbf{u}_{\mathbf{0}}\right)+i m\left(u_{1} \mathbf{v}, \mathbf{u}_{\mathbf{0}}\right), \forall(\mathbf{u}, \mathbf{v}) \in \mathcal{V}_{1 c} \times \mathcal{W}_{1 c}$,

(v) $c_{2 m}\left(\mathbf{u}, \rho_{0}, \phi\right)=-c_{2 m}\left(\mathbf{u}, \bar{\phi}, \rho_{0}\right)+i m\left(u_{1} \phi, \rho_{0}\right), \forall(\mathbf{u}, \mathbf{v}) \in \mathcal{V}_{1 c} \times \mathcal{V}_{2 c}$,

(vi) $\operatorname{Re}\left(b_{1 m}\left(\mathbf{u}_{\mathbf{0}}, \mathbf{v}, \mathbf{v}\right)\right)=0$ and $\operatorname{Re}\left(b_{2 m}\left(\mathbf{u}_{\mathbf{0}}, \phi, \phi\right)\right)=0, \forall(\mathbf{v}, \phi) \in \mathcal{W}_{1 c} \times \mathcal{V}_{2 c}$.

Notation: $\mathbf{u}=\left(u_{1}, u_{2}\right), \mathbf{u}_{\mathbf{0}}=\left(u_{0}, v_{0}\right), \overline{\mathbf{u}}$ is the complex conjugate of $\mathbf{u}$.

Proof. (i) (a1) $b_{1 m}\left(\mathbf{u}_{\mathbf{0}}, \mathbf{u}, \mathbf{v}\right)=i m\left(u_{0} \mathbf{u}, \mathbf{v}\right)+\left(v_{0} \frac{\partial \mathbf{u}}{\partial y}, \mathbf{v}\right)+\left(w_{0} \frac{\partial \mathbf{u}}{\partial z}, \mathbf{v}\right)$.

Since $\mathbf{u}_{\mathbf{0}}$ and $w_{0} \in L^{\infty}(\omega)$, then there exists a positive constant $C$ such that:

$$
\left|\left(\left(\mathbf{u}_{\mathbf{0}} \nabla_{m}\right) \mathbf{u}, \mathbf{v}\right)+\left(w_{0} \frac{\partial \mathbf{u}}{\partial z}, \mathbf{v}\right)\right| \leq C\|\mathbf{u}\|\|\mathbf{v}\|_{L_{c}^{2}(\omega)} .
$$

The same proof is valid for (a2).

(b1) $c_{1 m}\left(\mathbf{u}, \mathbf{u}_{\mathbf{0}}, \mathbf{v}\right)=\left(u_{2} \frac{\partial \mathbf{u}_{\mathbf{0}}}{\partial y}, \mathbf{v}\right)+\left(W_{m}(\mathbf{u}) \frac{\partial \mathbf{u}_{\mathbf{0}}}{\partial z}, \mathbf{v}\right)$.

Since $\nabla_{0} \mathbf{u}_{\mathbf{0}}$ and $\frac{\partial w_{0}}{\partial z} \in L^{\infty}(\omega)$, then there exists a positive constant $C$ such that:

$$
\left|c_{1 m}\left(\mathbf{u}, \mathbf{u}_{\mathbf{0}}, \mathbf{v}\right)\right| \leq C\|\mathbf{u}\|\|\mathbf{v}\|_{L_{c}^{2}(\omega)},
$$

according to the Proposition 3.1. The same proof is valid for (b2).

(ii) $b_{1 m}\left(\mathbf{u}_{\mathbf{0}}, \mathbf{u}, \mathbf{v}\right)=\left(\left(i m u_{0}+v_{0} \frac{\partial}{\partial y}+w_{0} \frac{\partial}{\partial z}\right) \mathbf{u}, \mathbf{v}\right)$. 
Since $\mathbf{u}_{\mathbf{0}}$ is real, we have

$$
\overline{b_{1 m}}\left(\mathbf{u}_{\mathbf{0}}, \mathbf{v}, \mathbf{u}\right)=\left(\left(-i m u_{0}+v_{0} \frac{\partial}{\partial y}+w_{0} \frac{\partial}{\partial z}\right) \overline{\mathbf{v}}, \overline{\mathbf{u}}\right)
$$

and then

$$
b_{1 m}\left(\mathbf{u}_{\mathbf{0}}, \mathbf{u}, \mathbf{v}\right)+\overline{b_{1 m}}\left(\mathbf{u}_{\mathbf{0}}, \mathbf{v}, \mathbf{u}\right)=\int_{\omega}\left(v_{0} \frac{\partial(\mathbf{u} \cdot \overline{\mathbf{v}})}{\partial y}+w_{0} \frac{\partial(\mathbf{u} \cdot \overline{\mathbf{v}})}{\partial z}\right) d \omega .
$$

Using Green's formula we obtain

$$
\begin{aligned}
b_{1 m}\left(\mathbf{u}_{\mathbf{0}}, \mathbf{u}, \mathbf{v}\right)+\overline{b_{1 m}}\left(\mathbf{u}_{\mathbf{0}}, \mathbf{v}, \mathbf{u}\right)= & -\int_{\omega}\left(\operatorname{div}_{0}\left(\mathbf{u}_{\mathbf{0}}\right)+\frac{\partial}{\partial z} w_{0}\right)(\mathbf{u} \cdot \overline{\mathbf{v}}) d \omega \\
& +\int_{\partial \omega}\left(\left(\mathbf{u}_{\mathbf{0}}, w_{0}\right) \cdot \mathbf{n}_{0}\right)(\mathbf{u} \cdot \overline{\mathbf{v}}) d \gamma
\end{aligned}
$$

Since $\mathbf{u}_{\mathbf{0}} \in \mathcal{V}_{0}$, then $\operatorname{div}_{0}\left(\mathbf{u}_{\mathbf{0}}\right)+\frac{\partial}{\partial z} w_{0}=0$, and $\left(\mathbf{u}_{\mathbf{0}}, w_{0}\right) \cdot \mathbf{n}_{0}=0$. We have then the result (ii). The same proof is valid for (iii).

(iv) According to the definition of $c_{1 m}$ we have

$$
c_{1 m}\left(\mathbf{u}, \mathbf{u}_{\mathbf{0}}, \mathbf{v}\right)=\left(\left(u_{2} \frac{\partial}{\partial y}+W_{m}(\mathbf{u}) \frac{\partial}{\partial z}\right) \mathbf{u}_{\mathbf{0}}, \mathbf{v}\right)
$$

and

$$
c_{1 m}\left(\mathbf{u}, \overline{\mathbf{v}}, \mathbf{u}_{\mathbf{0}}\right)=\left(\left(u_{2} \frac{\partial}{\partial y}+W_{m}(\mathbf{u}) \frac{\partial}{\partial z}\right) \overline{\mathbf{v}}, \mathbf{u}_{\mathbf{0}}\right) .
$$

Since $\mathbf{u}_{\mathbf{0}}$ is real, we have

$$
c_{1 m}\left(\mathbf{u}, \mathbf{u}_{\mathbf{0}}, \mathbf{v}\right)+c_{1 m}\left(\mathbf{u}, \overline{\mathbf{v}}, \mathbf{u}_{\mathbf{0}}\right)=\int_{\omega}\left(u_{2} \frac{\partial\left(\mathbf{u}_{\mathbf{0}} \cdot \overline{\mathbf{v}}\right)}{\partial y}+W_{m}(\mathbf{u}) \frac{\partial\left(\mathbf{u}_{\mathbf{0}} \cdot \overline{\mathbf{v}}\right)}{\partial z}\right) d \omega .
$$

According to $\mathbf{u} \in \mathcal{V}_{1 c}\left(u_{2}=0\right.$ on $\gamma_{3} \cup \gamma_{4}$ and $W_{m}(\mathbf{u})=0$ on $\left.\gamma_{0} \cup \gamma_{5}\right)$ and integrating by part with respect to $y$ (resp. $z$ ), we have

$$
c_{1 m}\left(\mathbf{u}, \mathbf{u}_{\mathbf{0}}, \mathbf{v}\right)+c_{1 m}\left(\mathbf{u}, \overline{\mathbf{v}}, \mathbf{u}_{\mathbf{0}}\right)=\int_{\omega}\left(-\frac{\partial u_{2}}{\partial y}\left(\mathbf{u}_{\mathbf{0}} \cdot \overline{\mathbf{v}}\right)-\frac{\partial W_{m}(\mathbf{u})}{\partial z}\left(\mathbf{u}_{\mathbf{0}} \cdot \overline{\mathbf{v}}\right)\right) d \omega .
$$

Since

$$
W_{m}(\mathbf{u})=-\int_{-H}^{z}\left(i m u_{1}+\frac{\partial u_{2}}{\partial y}\right) d z^{\prime}
$$

we thus obtain the result (iv). The same proof is valid for (v).

Setting $\mathbf{v}=\mathbf{u}$ (resp. $\phi=\rho$ ) in (iii) (resp. in (iv)) we deduce the result (vi). 


\subsection{Existence and uniqueness of the solution.}

Theorem 3.1. For $\left(\mathbf{u}_{\mathbf{0}}, w_{0}\right)$ and $\mathbf{f}$ given, $\left(\mathbf{u}_{\mathbf{0}}, w_{0}\right) \in \mathcal{V}_{0}, \mathbf{u}_{\mathbf{0}}, w_{0}, \nabla_{0} \mathbf{u}_{\mathbf{0}}, \frac{\partial w_{0}}{\partial z}$, $\rho_{0}, \nabla_{0} \rho_{0}, \frac{\partial \rho_{0}}{\partial z} \in L^{\infty}(\omega)$ and $\mathbf{f} \in L^{2}\left(0, T, L_{c}^{2}\left(\gamma_{0}\right)\right)$, there exists a unique solution $(\mathbf{u}, \rho)$ of problem (3.1) satisfying:

$$
\begin{gathered}
\mathbf{u} \in L^{2}\left(0, T, \mathcal{V}_{1 c}\right) \cap C^{0}\left([0, T], \mathcal{H}_{1 c}\right), \frac{\partial \mathbf{u}}{\partial t} \in L^{2}\left(0, T, \mathcal{V}_{1 c}^{\prime}\right), \\
\rho \in L^{2}\left(0, T, \mathcal{V}_{2}\right) \cap C^{0}\left([0, T], \mathcal{H}_{2 c}\right), \frac{\partial \rho}{\partial t} \in L^{2}\left(0, T, \mathcal{V}_{2 c}^{\prime}\right)
\end{gathered}
$$

Proof. To prove the existence of a solution we use the Faedo-Galerkin method with the same notations as in Section 2: $\left(\mathbf{v}_{1}, \ldots, \mathbf{v}_{l}, \ldots\right)$ is a total and free sequence in $\mathcal{V}_{1 c}$ and $\left(\phi_{1}, \ldots, \phi_{l}, \ldots\right)$ is a total and free sequence in $\mathcal{V}_{2 c}$. The approached solution $\left(\mathbf{u}_{l}, \rho_{l}\right)$ is defined as the solution of the following problem:

$$
\begin{gathered}
\left(\frac{\partial \mathbf{u}_{l}}{\partial t}, \mathbf{v}_{k}\right)+a_{1 m}\left(\mathbf{u}_{l}, \mathbf{v}_{k}\right)+L_{1 m}\left(\mathbf{u}_{l}, \rho_{l}, \mathbf{v}_{k}\right)=\left(\mathbf{f}, \gamma_{0} \mathbf{v}_{k}\right)_{\gamma_{0}}, \\
\left(\frac{\partial \rho_{l}}{\partial t}, \phi_{k}\right)+a_{2 m}\left(\rho_{l}, \phi_{k}\right)+l_{2 m}\left(\mathbf{u}_{l}, \rho_{l}, \phi_{k}\right)=0, \forall k=1, \ldots, l .
\end{gathered}
$$

By applying the coercivity of $a_{1 m}$ and $a_{2 m}$, the result of Propositions 3.1 and 3.2, we obtain the estimations:

$$
\begin{aligned}
& \frac{d}{d t}\left|\mathbf{u}_{l}\right|^{2}+2 \alpha_{1 m}\left\|\mathbf{u}_{l}\right\|_{W}^{2} \leq c_{1}\left|\mathbf{u}_{l}\right|\left\|\mathbf{u}_{l}\right\|+c_{2}\left|\rho_{l}\right|\left\|\mathbf{u}_{l}\right\|+c_{3}|\mathbf{f}|_{\gamma_{0}}\left\|\mathbf{u}_{l}\right\| \\
& \frac{d}{d t}\left|\rho_{l}\right|^{2}+2 \alpha_{2 m}\left\|\rho_{l}\right\|^{2} \leq c_{4}\left|\rho_{l}\right|\left\|\mathbf{u}_{l}\right\|+c_{5}\left\|\rho_{l}\right\|\left|\rho_{l}\right|
\end{aligned}
$$

where $\left(c_{i}\right)_{i=1, \ldots, 5}$ are positive constants. By setting $X_{l}=\left(\mathbf{u}_{l}, \rho_{l}\right)$, we can deduce from (3.4) the estimation

$$
\frac{d}{d t}\left|X_{l}\right|^{2}+K\left\|X_{l}\right\|_{W}^{2} \leq c_{6}\left|X_{l}\right|^{2}+c_{7}|\mathbf{f}|_{\gamma_{0}}^{2},
$$

where $K$ is a positive constant. Then the proof is achieved in the same way as in the proof of Theorem 2.1.

Proposition 3.3. If $(\mathbf{u}, \rho)$ is the solution of problem (3.1), then there is a unique distribution pressure $P$ (up to a constant) such that $(\mathbf{u}, \rho, P)$ is solution of problem (1.10), in the distribution sense.

The proof of this proposition is very similar to that of Proposition 2.2. 


\subsection{A result of regularity.}

From now on we assume the following regularity of the wind-stress:

$$
\mathbf{f} \in L^{2}\left(0, T, H_{c}^{1}\left(\gamma_{0}\right)\right), \frac{\partial \mathbf{f}}{\partial t} \in L^{2}\left(0, T, L_{c}^{2}\left(\gamma_{0}\right)\right),
$$

f must be consistent with the initial and boundary conditions (1.6), (1.11) imposed to the velocity $\mathbf{u}$. We then have to impose the following compatibility conditions:

$$
\begin{aligned}
& \mathbf{f}(0)=\mathbf{0} \text { on } \gamma_{0}, \\
& f_{2}\left(-L_{y}, t\right)=f_{2}\left(L_{y}, t\right)=0, \forall t \in[0, T] \\
& \frac{\partial f_{1}}{\partial y}\left(-L_{y}, t\right)=\frac{\partial f_{1}}{\partial y}\left(L_{y}, t\right)=0, \forall t \in[0, T] .
\end{aligned}
$$

Lemma 3.3. If the wind-stress $\mathbf{f}$ satisfies the conditions (3.6), (3.7), then $(\mathbf{u}, \rho)$, solution of problem $(3.1)$, is such that:

$$
\begin{aligned}
& (\mathbf{u}, \rho) \in \mathcal{C}^{0}\left([0, T], \mathcal{V}_{1 c} \times \mathcal{V}_{2 c}\right) \\
& \left(\frac{\partial \mathbf{u}}{\partial t}, \frac{\partial \rho}{\partial t}\right) \in L^{2}\left(0, T, \mathcal{V}_{1 c} \times \mathcal{V}_{2 c}\right) \cap \mathcal{C}^{0}\left([0, T], \mathcal{H}_{1 c} \times \mathcal{H}_{2 c}\right)
\end{aligned}
$$

Theorem 3.2. If the wind-stress $\mathbf{f}$ satisfies the regularity (3.6), and the initial and boundary conditions (3.7), then the solution $(\mathbf{u}, \rho, P)$ of problem (3.1) is such that

$$
\mathbf{u} \in L^{2}\left(0, T, H_{c}^{2}(\omega)\right), \rho \in L^{2}\left(0, T, H_{c}^{2}(\omega)\right), P \in L^{2}\left(0, T, L_{c}^{2}\left(\gamma_{0}\right)\right) .
$$

Proof. The proof of Lemma 3.2 and theorem 3.2 are very similar to that of Lemma 2.3 and Theorem 2.2. So we omit the details.

Proposition 3.4. If the wind-stress $\mathbf{f}$ satisfies the conditions (3.6), (3.7) and $\mathbf{u}_{\mathbf{0}}, \rho_{0}, w_{0}, \nabla_{0} \mathbf{u}_{\mathbf{0}}, \nabla_{0} \rho_{0}, \frac{\partial \mathbf{u}_{\mathbf{0}}}{\partial z}$ and $\frac{\partial \rho_{0}}{\partial z} \in L^{\infty}(\omega)$, then the solution $(\mathbf{u}, \rho, P)$ of problem (3.1) satisfies the following estimates:

(i) $\|\mathbf{u}\|_{L^{2}\left(0, T, H_{c}^{2}(\omega)\right)} \leq C\|\mathbf{f}\|_{\mathcal{U}_{c}}$,

(ii) $\|\rho\|_{L^{2}\left(0, T, H_{c}^{2}(\omega)\right)} \leq C\|\mathbf{f}\|_{\mathcal{U}_{c}}$,

(iii) $\|P\|_{L^{2}\left(0, T, L_{c}^{2}\left(\gamma_{0}\right)\right)} \leq C\|\mathbf{f}\|_{\mathcal{U}_{c}}$, where

$$
\begin{gathered}
\mathcal{U}_{c}=\left\{\mathbf{f} \mid \mathbf{f} \in L^{2}\left(0, T, H_{c}^{1}\left(\gamma_{0}\right)\right), \frac{\partial \mathbf{f}}{\partial t} \in L^{2}\left(0, T, L_{c}^{2}\left(\gamma_{0}\right)\right)\right\} \\
\|\mathbf{f}\|_{\mathcal{U}}^{2}=\|\mathbf{f}\|_{L^{2}\left(0, T, H_{c}^{1}\left(\gamma_{0}\right)\right)}^{2}+\left\|\frac{\partial \mathbf{f}}{\partial t}\right\|_{L^{2}\left(0, T, L_{c}^{2}\left(\gamma_{0}\right)\right)}^{2} .
\end{gathered}
$$


Proof. According to the Theorem 3.2, the estimation (2.12) and the regularity of $\left(\mathbf{u}_{\mathbf{0}}, w_{0}, \rho_{0}\right)$ we have:

$$
\begin{aligned}
& \|\mathbf{u}\|_{L^{2}\left(0, T, H_{c}^{2}(\omega)\right)}^{2} \leq C\left(\|\mathbf{u}\|_{L^{2}\left(0, T, \mathcal{V}_{1 c}\right)}^{2}+\left\|\frac{\partial \mathbf{u}}{\partial t}\right\|_{L^{2}\left(0, T, \mathcal{H}_{1 c}\right)}^{2}\right. \\
& \left.+\|\rho\|_{L^{2}\left(0, T, \mathcal{H}_{2 c}\right)}^{2}+\|\mathbf{f}\|_{L^{2}\left(0, T, H_{c}^{1}\left(\gamma_{0}\right)\right)}^{2}\right), \\
& \|\rho\|_{L^{2}\left(0, T, H_{c}^{2}(\omega)\right)} \leq C\left(\|\rho\|_{L^{2}\left(0, T, \mathcal{V}_{2 c}\right)}^{2}+\left\|\frac{\partial \rho}{\partial t}\right\|_{L^{2}\left(0, T, \mathcal{H}_{2 c}\right)}^{2}\right. \\
& \left.\quad+\|\mathbf{u}\|_{L^{2}\left(0, T, \mathcal{H}_{1 c}\right)}^{2}\right), \\
& \|P\|_{L^{2}\left(0, T, L_{c}^{2}(\gamma)\right)} \leq C\left(\|\mathbf{u}\|_{L^{2}\left(0, T, \mathcal{V}_{1 c}\right)}^{2}+\left\|\frac{\partial \mathbf{u}}{\partial t}\right\|_{L^{2}\left(0, T, \mathcal{H}_{1 c}\right)}^{2}\right. \\
& \left.\quad+\|\rho\|_{L^{2}\left(0, T, \mathcal{H}_{2 c}\right)}^{2}+\|\mathbf{f}\|_{L^{2}\left(0, T, H_{c}^{1}\left(\gamma_{0}\right)\right)}^{2}\right) .
\end{aligned}
$$

Setting $\mathbf{v}=\mathbf{u}$ and $\phi=\rho$ in (3.1) gives

$$
\begin{aligned}
& \frac{d}{d t}|\mathbf{u}|^{2}+2 a_{1 m}(\mathbf{u}, \mathbf{u})+L_{1 m}(\mathbf{u}, \rho, \mathbf{u})=2\left(\mathbf{f}, \gamma_{0} \mathbf{u}\right)_{\gamma_{0}}, \\
& \frac{d}{d t}|\rho|^{2}+2 a_{2 m}(\rho, \rho)+l_{2 m}(\mathbf{u}, \rho, \rho)=0
\end{aligned}
$$

According to the coercivity of $a_{1 m}$ and $a_{2 m}$, the result of Propositions 3.1 and 3.2, and by applying Hölder's inequality, we deduce that:

$$
\frac{d}{d t}|X|^{2}+K\|X\|_{W}^{2} \leq c_{9}|X|^{2}+c_{7}|\mathbf{f}|_{\gamma_{0}}^{2}
$$

where $X=(\mathbf{u}, \rho), K$ is a positive constant. Applying the Gronwall lemma now gives

$$
|X|(t)^{2} \leq C\|\mathbf{f}\|_{L^{2}\left(0, T, H_{c}^{1}\left(\gamma_{0}\right)\right)}^{2}, \quad \forall t \in[0, T] .
$$

We deduce from (3.9), (3.10) that

$$
\begin{gathered}
\|X\|_{L^{2}\left(0, T, \mathcal{H}_{1 c} \times \mathcal{H}_{2 c}\right)}^{2} \leq C\|\mathbf{f}\|_{L^{2}\left(0, T, H_{c}^{1}\left(\gamma_{0}\right)\right)}^{2}, \\
\|X\|_{L^{2}\left(0, T, \mathcal{V}_{1 c} \times \mathcal{V}_{2 c}\right)}^{2} \leq C\|\mathbf{f}\|_{L^{2}\left(0, T, H_{c}^{1}\left(\gamma_{0}\right)\right)}^{2} .
\end{gathered}
$$

Setting $(\mathbf{v}, \phi)=\left(\frac{\partial \mathbf{u}}{\partial t}, \frac{\partial \rho}{\partial t}\right)=\left(\mathbf{u}^{\prime}, \phi^{\prime}\right)$ in $(3.1)$ gives

$$
\begin{aligned}
& \left|\mathbf{u}^{\prime}\right|^{2}+a_{1 m}\left(\mathbf{u}, \mathbf{u}^{\prime}\right)+L_{1 m}\left(\mathbf{u}, \rho, \mathbf{u}^{\prime}\right)=\left(\mathbf{f}, \gamma_{0} \mathbf{u}^{\prime}\right)_{\gamma_{0}}, \\
& \left|\rho^{\prime}\right|^{2}+a_{2 m}\left(\rho, \rho^{\prime}\right)+l_{2 m}\left(\mathbf{u}, \rho, \rho^{\prime}\right)=0
\end{aligned}
$$

and

$$
\begin{aligned}
& \left|\mathbf{u}^{\prime}\right|^{2}+\overline{a_{1 m}}\left(\mathbf{u}, \mathbf{u}^{\prime}\right)+\overline{L_{1 m}}\left(\mathbf{u}, \rho, \mathbf{u}^{\prime}\right)=\overline{\left(\mathbf{f}, \gamma_{0} \mathbf{u}^{\prime}\right)_{\gamma_{0}}}, \\
& \left|\rho^{\prime}\right|^{2}+\overline{a_{2 m}}\left(\rho, \rho^{\prime}\right)+\overline{l_{2 m}}\left(\mathbf{u}, \rho, \rho^{\prime}\right)=0 .
\end{aligned}
$$


Summing these two systems we obtain:

$$
\begin{aligned}
& 2\left|\mathbf{u}^{\prime}\right|^{2}+\frac{d}{d t}\left(a_{1 m}(\mathbf{u}, \mathbf{u})\right)+2 \operatorname{Re}\left(L_{1 m}\left(\mathbf{u}, \rho, \mathbf{u}^{\prime}\right)\right)=\left(\overline{\mathbf{f}}, \gamma_{0} \overline{\mathbf{u}^{\prime}}\right)_{\gamma_{0}}+\left(\mathbf{f}, \gamma_{0} \mathbf{u}^{\prime}\right)_{\gamma_{0}}, \\
& 2\left|\rho^{\prime}\right|^{2}+\frac{d}{d t}\left(a_{2 m}(\rho, \rho)\right)+2 \operatorname{Re}\left(l_{2 m}\left(\mathbf{u}, \rho, \rho^{\prime}\right)\right)=0 .
\end{aligned}
$$

Moreover

$$
\left(\overline{\mathbf{f}}, \gamma_{0} \overline{\mathbf{u}^{\prime}}\right)_{\gamma_{0}}+\left(\mathbf{f}, \gamma_{0} \mathbf{u}^{\prime}\right)_{\gamma_{0}}=2 \frac{d}{d t}\left(\operatorname{Re}\left(\left(\mathbf{f}, \gamma_{0} \mathbf{u}\right)_{\gamma_{0}}\right)\right)-2 \operatorname{Re}\left(\left(\mathbf{f}^{\prime}, \gamma_{0} \mathbf{u}\right)_{\gamma_{0}}\right) .
$$

Then after integration with respect to time we obtain

$$
\begin{aligned}
& 2 \int_{0}^{t}\left|\mathbf{u}^{\prime}\right|^{2}(s) d s+a_{1 m}(\mathbf{u}(t), \mathbf{u}(t))=-2 \int_{0}^{t} \operatorname{Re}\left(L_{1 m}\left(\mathbf{u}(s), \rho(s), \mathbf{u}^{\prime}(s)\right)\right) d s \\
& \quad+2 \operatorname{Re}\left(\left(\mathbf{f}(t), \gamma_{0} \mathbf{u}(t)\right)_{\gamma_{0}}\right)-2 \int_{0}^{t} \operatorname{Re}\left(\left(\mathbf{f}^{\prime}(s), \gamma_{0} \mathbf{u}(s)\right)_{\gamma_{0}}\right) d s \\
& 2 \int_{0}^{t}\left|\rho^{\prime}\right|^{2}(s) d s+a_{2 m}(\rho(t), \rho(t))=-2 \int_{0}^{t} \operatorname{Re}\left(l_{2 m}\left(\mathbf{u}(s), \rho(s), \rho^{\prime}(s)\right)\right) d s \\
& \forall t \in[0, T] .
\end{aligned}
$$

Applying the coercivity of $a_{1 m}$ and $a_{2 m}$ yields

$$
\begin{aligned}
& 2 \int_{0}^{t}\left|\mathbf{u}^{\prime}\right|^{2}(s) d s+\alpha_{1 m}\|\mathbf{u}\|^{2} \leq 2 \int_{0}^{t}\left|L_{1 m}\left(\mathbf{u}(s), \rho(s), \mathbf{u}^{\prime}(s)\right)\right| d s \\
& \quad+2\left|\left(\mathbf{f}(t), \gamma_{0} \mathbf{u}(t)\right)_{\gamma_{0}}\right|+2 \int_{0}^{t}\left|\left(\mathbf{f}^{\prime}(s), \gamma_{0} \mathbf{u}(s)\right)_{\gamma_{0}}\right| d s \\
& 2 \int_{0}^{t}\left|\rho^{\prime}\right|^{2}(s) d s+\alpha_{2 m}\|\rho\|^{2} \leq 2 \int_{0}^{t}\left|l_{2 m}\left(\mathbf{u}(s), \rho(s), \rho^{\prime}(s)\right)\right| d s \\
& \forall t \in[0, T] .
\end{aligned}
$$

According to the results of the Propositions 3.1 and 3.2, which yields:

$$
\begin{aligned}
& \left|L_{1 m}\left(\mathbf{u}, \rho, \mathbf{u}^{\prime}\right)\right| \leq C_{1}(\|\mathbf{u}\|+|\rho|)\left|\mathbf{u}^{\prime}\right|, \\
& \left|l_{2 m}\left(\mathbf{u}, \rho, \rho^{\prime}\right)\right| \leq C_{2}(\|\rho\|+|\mathbf{u}|)\left|\rho^{\prime}\right|
\end{aligned}
$$

Since $\mathbf{f}(t)=\int_{0}^{t} \mathbf{f}^{\prime}(s) d s$, we have

$$
|\mathbf{f}|_{\gamma_{0}}^{2}(t) \leq C\left\|\mathbf{f}^{\prime}\right\|_{L^{2}\left(0, T, L_{c}^{2}\left(\gamma_{0}\right)\right)}^{2}, \forall t \in[0, T] .
$$

According to (3.11)-(3.13) we obtain

$$
\begin{aligned}
& \left\|\mathbf{u}^{\prime}\right\|_{L^{2}\left(0, T, \mathcal{H}_{1 c}\right)}^{2} \leq C\|\mathbf{f}\|_{\mathcal{U}_{c}}^{2}, \\
& \left\|\rho^{\prime}\right\|_{L^{2}\left(0, T, \mathcal{H}_{2 c}\right)}^{2} \leq C\|\mathbf{f}\|_{\mathcal{U}_{c}}^{2} .
\end{aligned}
$$

We deduce from (3.8), (3.11) and (3.14) the estimates (i)-(iii). 


\subsection{The optimal control problem.}

The problem is controlled by the variability of the wind-stress $\mathbf{f}$. The observation is the pressure on $\gamma_{0}$, deduced from altimetric measurements. Controls and observations are thus defined on $\gamma_{0}$. Thereby

$$
\mathcal{U}_{c}=\left\{\mathbf{f} \mid \mathbf{f} \in L^{2}\left(0, T, H_{c}^{1}\left(\gamma_{0}\right)\right), \frac{\partial \mathbf{f}}{\partial t} \in L^{2}\left(0, T, L_{c}^{2}\left(\gamma_{0}\right)\right)\right\}
$$

will be the control space; $\mathcal{B}_{c}=L^{2}\left(0, T, L_{c}^{2}\left(\gamma_{0}\right)\right)$ will be the observation space. For each control $\mathbf{f},(\mathbf{u}(\mathbf{f}), \rho(\mathbf{f}), P(\mathbf{f}))$ is the solution of the weak problem (3.2), and the cost function $\mathcal{J}$ is defined by

$$
\mathcal{J}(\mathbf{f})=\frac{1}{2}\left\|P(\mathbf{f})-P_{o b s}\right\|_{\mathcal{B}_{c}}^{2}+\frac{\alpha}{2}\|\mathbf{f}\|_{\mathcal{U}_{c}}^{2}
$$

$P_{\text {obs }} \in \mathcal{B}_{c}$ is the observation. $\alpha$ is a given positive constant $(\alpha \neq 0)$. The optimal control problem then is as follows:

$$
\begin{aligned}
& \text { Find } \mathbf{f} \in \mathcal{U}_{c} \text { such that } \\
& \mathcal{J}(\mathbf{f})=\inf _{\mathbf{g} \in \mathcal{U}_{c}} \mathcal{J}(\mathbf{g}) .
\end{aligned}
$$

\section{Remark 3.2.}

1) The derivative of $\mathcal{J}$, at point $\mathbf{f}$, in $\mathbf{g}$ direction is given by

$$
\left(\mathcal{J}^{\prime}(\mathbf{f}), \mathbf{g}\right)=\operatorname{Re}\left(\left(P(\mathbf{f})-P_{o b s}, P(\mathbf{g})\right)_{\mathcal{B}_{c}}+\alpha(\mathbf{f}, \mathbf{g})_{\mathcal{U}_{c}}\right), \forall(\mathbf{f}, \mathbf{g}) \in \mathcal{U}_{c}^{2} .
$$

2) $P \in L_{c}^{2}\left(\gamma_{0}\right)$ and $P$ is defined regardless of any time-dependant function. We now determine this function by setting the condition

$$
\int_{\gamma_{0}} P d \gamma=\int_{\gamma_{0}} P_{o b s} d \gamma
$$

Proposition 3.5. Problem (3.16) admits one unique solution $\mathbf{f} \in \mathcal{U}_{c}$.

The proof of this proposition is very similar to that of Proposition 2.5.

For a wind-stress $\mathbf{f} \in \mathcal{U}_{c},(\mathbf{u}(\mathbf{f}), \rho(\mathbf{f}), P(\mathbf{f}))$ is the solution of (3.2). We note $(\tilde{\mathbf{u}}(\mathbf{f}), \tilde{\rho}(\mathbf{f}), \tilde{P}(\mathbf{f}))$ the adjoint state which is the solution of the adjoint 
problem:

$$
\begin{aligned}
& \left(-\frac{\partial \mathbf{u}^{*}(\mathbf{f})}{\partial t}, \mathbf{v}\right)+a_{1 m}\left(\mathbf{u}^{*}(\mathbf{f}), \mathbf{v}\right)+\overline{l_{1 m}}\left(\mathbf{v}, \mathbf{u}^{*}(\mathbf{f})\right)+\left(\rho^{*}(\mathbf{f}) \nabla_{0} \rho_{0}, \mathbf{v}\right) \\
& \quad-\left(\rho^{*}(\mathbf{f}) \frac{\partial \rho_{0}}{\partial z}, \int_{-H}^{z} \operatorname{div}_{m}(\mathbf{v}) d z^{\prime}\right)+\frac{1}{\rho_{m o y}}\left(P^{*}(\mathbf{f}), \int_{-H}^{0} \operatorname{div}_{m}(\mathbf{v}) d z^{\prime}\right)_{\gamma_{0}} \\
& \quad-\left(\nu_{1 v} \int_{-H}^{0} \operatorname{div}_{m}\left(\mathbf{u}^{*}(\mathbf{f})\right) d z^{\prime}, \gamma_{0} \operatorname{div}_{m}(\mathbf{v})\right)_{\gamma_{0}}-d\left(\mathbf{u}^{*}(\mathbf{f}), \mathbf{v}\right)=0, \\
& \left(-\frac{\partial \rho^{*}(\mathbf{f})}{\partial t}, \phi\right)+a_{2 m}\left(\rho^{*}(\mathbf{f}), \phi\right)-\left(\left(\mathbf{u}_{\mathbf{0}} \nabla_{m}\right) \rho^{*}(\mathbf{f}), \phi\right)-\left(w_{0} \frac{\partial \rho^{*}(\mathbf{f})}{\partial z}, \phi\right) \\
& \quad-\frac{g}{\rho_{m o y}}\left(\int_{-H}^{z} \operatorname{div}_{m}\left(\mathbf{u}^{*}(\mathbf{f})\right) d z^{\prime}, \phi\right)=0, \\
& \frac{1}{\rho_{m o y}}\left(\int_{-H}^{0} \operatorname{div}_{m}\left(\mathbf{u}^{*}(\mathbf{f})\right) d z^{\prime}, Q\right)_{\gamma_{0}}=\left(P(\mathbf{f})-P_{o b s}, Q\right)_{\gamma_{0}}, \\
& \forall(\mathbf{v}, \phi, Q) \in \mathcal{W}_{1 c} \cap H_{c}^{2} \times \mathcal{V}_{2 c} \cap H_{c}^{2} \times L_{c}^{2}\left(\gamma_{0}\right), \\
& \mathbf{u}^{*}(\mathbf{f})(T)=\mathbf{0}, \rho^{*}(\mathbf{f})(T)=0, \\
& \int_{\gamma_{0}}\left(P(\mathbf{f})-P_{o b s}\right) d \gamma_{0}=0 .
\end{aligned}
$$

Proposition 3.6. The adjoint problem (3.17) admits one unique solution such that:

$$
(\tilde{\mathbf{u}}(\mathbf{f}), \tilde{\rho}(\mathbf{f}), \tilde{P}(\mathbf{f})) \in L^{2}\left(0, T, \mathcal{W}_{1 c} \times \mathcal{V}_{2 c} \times L_{c}^{2}\left(\gamma_{0}\right)\right)
$$

The proof of this proposition is very similar to that of Proposition 2.6.

Proposition 3.7. $\mathcal{J}^{\prime}(\mathbf{f})=0$ if and only if $\alpha \mathbf{f}-\Lambda^{-1} \gamma_{0} \mathbf{u}^{*}(\mathbf{f})=0$ in $\mathcal{U}_{c}$. $\lambda$ is the canonical isomorphism $\mathcal{U}_{c} \longrightarrow \mathcal{U}_{c}^{\prime}$ such that

$$
\langle\mathbf{g}, \mathbf{v}\rangle_{\mathcal{U}_{c}, \mathcal{U}_{c}^{\prime}}=(\Lambda \mathbf{g}, \mathbf{v})_{\mathcal{U}_{c}^{\prime}}=\left(\mathbf{g}, \Lambda^{-1} \mathbf{v}\right)_{\mathcal{U}_{c}}, \forall(\mathbf{g}, \mathbf{v}) \in \mathcal{U}_{c} \times \mathcal{U}_{c}^{\prime}
$$

Proof. $\left(\mathcal{J}^{\prime}(\mathbf{f}), \mathbf{g}\right)=\operatorname{Re}\left(\left(\left(P(\mathbf{f})-P_{o b s}, P(\mathbf{g})\right)_{\mathcal{B}_{c}}+\alpha(\mathbf{f}, \mathbf{g}) \mathcal{U}_{c}\right), \forall(\mathbf{f}, \mathbf{g})\right) \in \mathcal{U}_{c}^{2}$. The third part of equation (3.17) implies

$$
\left(\mathcal{J}^{\prime}(\mathbf{f}), \mathbf{g}\right)=\operatorname{Re}\left(\alpha(\mathbf{f}, \mathbf{g})_{\mathcal{U}_{c}}+\left(\int_{-H}^{0} \operatorname{div}_{m}\left(\mathbf{u}^{*}(\mathbf{f})\right) d z^{\prime}, P(\mathbf{g})\right)_{\mathcal{B}_{c}}\right), \forall(\mathbf{f}, \mathbf{g}) \in \mathcal{U}_{c}^{2}
$$


Setting $(\mathbf{v}, \phi, Q)=\left(\mathbf{u}^{*}(\mathbf{f}), \rho^{*}(\mathbf{f}), P^{*}(\mathbf{f})\right)$ in problem (3.2) gives (according to the definition of $\left.L_{1 m}\right)$ :

$$
\begin{aligned}
& \left(\frac{\partial \mathbf{u}(\mathbf{g})}{\partial t}, \mathbf{u}^{*}(\mathbf{f})\right)+a_{1 m}\left(\mathbf{u}(\mathbf{g}), \mathbf{u}^{*}(\mathbf{f})\right)+l_{1 m}\left(\mathbf{u}(\mathbf{g}), \mathbf{u}^{*}(\mathbf{f})\right) \\
& \quad+\frac{g}{\rho_{m o y}}\left(\rho(\mathbf{g}), \int_{-H}^{z} \operatorname{div}_{m}\left(\mathbf{u}^{*}(\mathbf{f})\right) d z^{\prime}\right) \\
& \quad-\left(\nu_{1 v} \gamma_{0}\left(\operatorname{div}_{m}(\mathbf{u}(\mathbf{g}))\right), \int_{-H}^{0} \operatorname{div}_{m}\left(\mathbf{u}^{*}(\mathbf{f})\right) d z^{\prime}\right)_{\gamma_{0}} \\
& \quad-\frac{1}{\rho_{m o y}}\left(P(\mathbf{g}), \int_{-H}^{0} \operatorname{div}_{m}\left(\mathbf{u}^{*}(\mathbf{f})\right) d z^{\prime}\right)_{\gamma_{0}}+d\left(\mathbf{u}(\mathbf{g}), \mathbf{u}^{*}(\mathbf{f})\right)=\left(\mathbf{g}, \gamma_{0} \mathbf{u}^{*}(\mathbf{f})\right)_{\gamma_{0}}, \\
& \left(\frac{\partial \rho(\mathbf{g})}{\partial t}, \rho^{*}(\mathbf{f})\right)+a_{2 m}\left(\rho(\mathbf{g}), \rho^{*}(\mathbf{f})\right)+l_{2 m}\left(\mathbf{u}(\mathbf{g}), \rho(\mathbf{g}), \rho^{*}(\mathbf{f})\right)=0 \\
& -\left(\int_{-H}^{0} \operatorname{div}_{m}(\mathbf{u}(\mathbf{g})) d z^{\prime}, P^{*}(\mathbf{f})\right)_{\gamma_{0}}=0 \\
& \mathbf{u}(\mathbf{g})(0)=\mathbf{0}, \rho(\mathbf{g})(0)=0 .
\end{aligned}
$$

By the time integration we then obtain:

$$
\begin{aligned}
& -\int_{0}^{T} \overline{\left(\frac{\partial \mathbf{u}^{*}(\mathbf{f})}{\partial t}, \mathbf{u}(\mathbf{g})\right)} d t+\int_{0}^{T} \overline{a_{1 m}\left(\mathbf{u}^{*}(\mathbf{f}), \mathbf{u}(\mathbf{g})\right)} d t \\
& +\int_{0}^{T} l_{1 m}\left(\mathbf{u}(\mathbf{g}), \mathbf{u}^{*}(\mathbf{f})\right)(t) d t+\frac{g}{\rho_{\text {moy }}} \int_{0}^{T}\left(\rho(\mathbf{g}), \int_{-H}^{z} \operatorname{div}_{m}\left(\mathbf{u}^{*}(\mathbf{f})\right) d z^{\prime}\right)(t) d t \\
& -\int_{0}^{T}\left(\nu_{1 v} \gamma_{0}\left(\operatorname{div}_{m}(\mathbf{u}(\mathbf{g}))\right), \int_{-H}^{0} \operatorname{div}_{m}\left(\mathbf{u}^{*}(\mathbf{f})\right) d z^{\prime}\right)_{\gamma_{0}}(t) d t \\
& -\frac{1}{\rho_{\text {moy }}} \int_{0}^{T}\left(P(\mathbf{g}), \int_{-H}^{0} \operatorname{div}_{m}\left(\mathbf{u}^{*}(\mathbf{f})\right) d z^{\prime}\right)_{\gamma_{0}}(t) d t+\int_{0}^{T} d\left(\mathbf{u}(\mathbf{g}), \mathbf{u}^{*}(\mathbf{f})\right)(t) d t \\
& =\int_{0}^{T}\left(\mathbf{g}, \gamma_{0} \mathbf{u}^{*}(\mathbf{f})\right)_{\gamma_{0}}(t) d t \\
& -\int_{0}^{T} \overline{\left(\frac{\partial \rho^{*}(\mathbf{f})}{\partial t}, \rho(\mathbf{g})\right)} d t+\int_{0}^{T} \overline{a_{2 m}\left(\rho^{*}(\mathbf{f}), \rho(\mathbf{g})\right)} d t \\
& -\int_{0}^{T} l_{2 m}\left(\mathbf{u}(\mathbf{g}), \rho(\mathbf{g}), \rho^{*}(\mathbf{f})\right)(t) d t=0, \\
& -\int_{0}^{T}\left(\int_{-H}^{0} \operatorname{div}_{m}(\mathbf{u}(\mathbf{g})) d z^{\prime}, P^{*}(\mathbf{f})\right)_{\gamma_{0}}(t) d t=0 .
\end{aligned}
$$


So,

$$
\begin{aligned}
& -\int_{0}^{T}\left(\frac{\partial \mathbf{u}^{*}(\mathbf{f})}{\partial t}, \mathbf{u}(\mathbf{g})\right) d t+\int_{0}^{T} a_{1 m}\left(\mathbf{u}^{*}(\mathbf{f}), \mathbf{u}(\mathbf{g})\right) d t \\
& +\int_{0}^{T} \overline{l_{1 m}}\left(\mathbf{u}(\mathbf{g}), \mathbf{u}^{*}(\mathbf{f})\right)(t) d t+\frac{g}{\rho_{m o y}} \int_{0}^{T}\left(\int_{-H}^{z} \operatorname{div}_{m}\left(\mathbf{u}^{*}(\mathbf{f})\right) d z^{\prime}, \rho(\mathbf{g})\right)(t) d t \\
& -\int_{0}^{T}\left(\nu_{1 v} \int_{-H}^{0} \operatorname{div}_{m}\left(\mathbf{u}^{*}(\mathbf{f})\right) d z^{\prime}, \gamma_{0}\left(\operatorname{div}_{m}(\mathbf{u}(\mathbf{g}))\right)\right)_{\gamma_{0}}(t) d t \\
& -\frac{1}{\rho_{m o y}} \int_{0}^{T}\left(\int_{-H}^{0} \operatorname{div}_{m}\left(\mathbf{u}^{*}(\mathbf{f})\right) d z^{\prime}, P(\mathbf{g})\right)_{\gamma_{0}}(t) d t \\
& -\int_{0}^{T} \bar{d}\left(\mathbf{u}^{*}(\mathbf{f}), \mathbf{u}(\mathbf{g})\right)(t) d t=\int_{0}^{T}\left(\gamma_{0} \mathbf{u}^{*}(\mathbf{f}), \mathbf{g}\right)_{\gamma_{0}}(t) d t \\
& -\int_{0}^{T}\left(\frac{\partial \rho^{*}(\mathbf{f})}{\partial t}, \rho(\mathbf{g})\right) d t+\int_{0}^{T} a_{2 m}\left(\rho^{*}(\mathbf{f}), \rho(\mathbf{g})\right) d t \\
& -\int_{0}^{T} \overline{l_{2 m}}\left(\mathbf{u}(\mathbf{g}), \rho(\mathbf{g}), \rho^{*}(\mathbf{f})\right)(t) d t=0 \\
& -\int_{0}^{T}\left(\int_{-H}^{0} \operatorname{div}_{m}(\mathbf{u}(\mathbf{g})) d z^{\prime}, P^{*}(\mathbf{f})\right)_{\gamma_{0}}(t) d t=0 .
\end{aligned}
$$

Since $\left(\mathbf{u}^{*}(\mathbf{f}), \rho^{*}(\mathbf{f}), P^{*}(\mathbf{f})\right)$ is the solution of the adjoint problem (3.17). We thus obtain

$$
\begin{aligned}
& -\int_{0}^{T}\left(\rho^{*}(\mathbf{f}) \frac{\partial \rho_{0}}{\partial z}, \int_{-H}^{z} \operatorname{div}_{m}(\mathbf{u}(\mathbf{g})) d z^{\prime}\right)(t) d t+\int_{0}^{T}\left(\left(\rho^{*}(\mathbf{f}) \nabla_{0}\right) \rho_{0}, \mathbf{u}(\mathbf{g})\right) d t \\
& =\frac{g}{\rho_{\text {moy }}} \int_{0}^{T}\left(\int_{-H}^{z} \operatorname{div}_{m}\left(\mathbf{u}^{*}(\mathbf{f})\right) d z^{\prime}, \rho(\mathbf{g})\right)(t) d t
\end{aligned}
$$

and

$$
\int_{0}^{T}\left(P(\mathbf{f})-P_{o b s}, P(\mathbf{g})\right)_{\gamma_{0}}=\int_{0}^{T}\left(\gamma_{0} \mathbf{u}^{*}(\mathbf{f}), \mathbf{g}\right) d t
$$

We can now conclude that $\mathcal{J}^{\prime}(\mathbf{f})=0$ if and only if

$$
\alpha \mathbf{f}-\Lambda^{-1} \gamma_{0} \mathbf{u}^{*}(\mathbf{f})=0 \text { in } \mathcal{U}_{c}
$$


We have proved that the optimal control $\mathbf{f}$, the solution of problem (3.16), is characterized by the following set of equations:

$$
\begin{aligned}
& \left(\frac{\partial \mathbf{u}(\mathbf{f})}{\partial t}, \mathbf{v}\right)+a_{1 m}(\mathbf{u}(\mathbf{f}), \mathbf{v})+l_{1 m}(\mathbf{u}(\mathbf{f}), \mathbf{v})+d(\mathbf{u}(\mathbf{f}), \mathbf{v}) \\
& +\frac{g}{\rho_{\text {moy }}}\left(\rho(\mathbf{f}), \int_{-H}^{z} \operatorname{div}(\mathbf{v}) d z^{\prime}\right)-\left(\nu_{1 v} \gamma_{0}\left(\operatorname{div}_{m}(\mathbf{u}(\mathbf{f}))\right), \int_{-H}^{0} \operatorname{div}_{m}(\mathbf{v}) d z^{\prime}\right) \\
& +\frac{1}{\rho_{\text {moy }}}\left(\int_{-H}^{0} \operatorname{div}_{m}(\mathbf{v}) d z^{\prime}, P(\mathbf{f})\right)_{\gamma_{0}}=\left(\mathbf{f}, \gamma_{0} \mathbf{v}\right) \\
& \left(\frac{\partial \rho(\mathbf{g})}{\partial t}, \phi\right)+a_{2 m}(\rho(\mathbf{g}), \phi)+l_{2 m}(\mathbf{u}(\mathbf{g}), \rho(\mathbf{g}), \phi)=0, \\
& \left(\int_{-H}^{0} \operatorname{div}(\mathbf{u}(\mathbf{g})) d z^{\prime}, Q\right)_{\gamma_{0}}=0 \text {, } \\
& \left(-\frac{\partial \mathbf{u}^{*}(\mathbf{f})}{\partial t}, \mathbf{v}\right)+a_{1 m}\left(\mathbf{u}^{*}(\mathbf{f}), \mathbf{v}\right)+\overline{l_{1 m}}\left(\mathbf{v}, \mathbf{u}^{*}(\mathbf{f})\right)+\left(\rho^{*}(\mathbf{f}) \nabla_{0} \rho_{0}, \mathbf{v}\right) \\
& -\left(\rho^{*}(\mathbf{f}) \frac{\partial \rho_{0}}{\partial z}, \int_{-H}^{z} \operatorname{div}_{m}(\mathbf{v}) d z^{\prime}\right)+\frac{1}{\rho_{\text {moy }}}\left(P^{*}(\mathbf{f}), \int_{-H}^{0} \operatorname{div}_{m}(\mathbf{v})\right)_{\gamma_{0}} \\
& -\left(\nu_{1 v} \int_{-H}^{0} \operatorname{div}_{m}\left(\mathbf{u}^{*}(\mathbf{f})\right) d z^{\prime}, \gamma_{0} \operatorname{div}_{m}(\mathbf{v})\right)_{\gamma_{0}}-d\left(\mathbf{u}^{*}(\mathbf{f}), \mathbf{v}\right)=0, \\
& \left(-\frac{\partial \rho^{*}(\mathbf{f})}{\partial t}, \phi\right)+a_{2 m}\left(\rho^{*}(\mathbf{f}), \phi\right)-\left(\left(\mathbf{u}_{\mathbf{0}} \nabla_{m}\right) \rho^{*}(\mathbf{f}), \phi\right)-\left(w_{0} \frac{\partial \rho^{*}(\mathbf{f})}{\partial z}, \phi\right) \\
& -\frac{g}{\rho_{\text {moy }}}\left(\int_{-H}^{z} \operatorname{div}_{m}\left(\mathbf{u}^{*}(\mathbf{f})\right) d z^{\prime}, \phi\right)=0, \\
& \frac{1}{\rho_{\text {moy }}}\left(\int_{-H}^{0} \operatorname{div}_{m}\left(\mathbf{u}^{*}(\mathbf{f})\right) d z^{\prime}, Q\right)_{\gamma_{0}}=\left(P(\mathbf{f})-P_{o b s}, Q\right)_{\gamma_{0}} \text {, } \\
& \forall(\mathbf{v}, \phi, Q) \in \mathcal{W}_{1 c} \cap H_{c}^{2} \times \mathcal{V}_{2 c} \cap H_{c}^{2} \times L_{c}^{2}\left(\gamma_{0}\right), \\
& \int_{\gamma_{0}}\left(P(\mathbf{f})-P_{o b s}\right) d \gamma_{0}=0 \text {. } \\
& \mathbf{u}^{*}(\mathbf{f})(T)=\mathbf{u}(\mathbf{f})(0)=\mathbf{0}, \rho^{*}(\mathbf{f})(T)=\rho(\mathbf{f})(0)=0, \\
& \alpha \mathbf{f}-\Lambda^{-1} \gamma_{0} \mathbf{u}^{*}(\mathbf{f})=0 \text { in } \mathcal{U}_{c} \text {. }
\end{aligned}
$$

\section{References}

[1] Adams, R. A., Sobolev Spaces, Academic Press, New York, 1975.

[2] Agmon, S., Douglis, A., Nirenberg, L., Estimates near the boundary for solutions of elliptic partial differential equations satisfying general boundary conditions II, Comm. Pure Appl. Math. 17 (1964), 35-92.

[3] Belmiloudi, A., Resolution of optimal control problem for a perturbation linearized Navier-Stokes type equations, Application of mathematics in engineering and economics (Sozopol), Proc. XXII Summer School (1996), Tech. Univ. Sofia, 39-51. 
[4] Belmiloudi, A., Method of characteristics and error estimates of the perturbation of a given mean flow, Applications of mathematics in engineering and economics (Sozopol), Proc. XXII Summer School (1996), Tech. Univ. Sofia, 25-38.

[5] Belmiloudi, A., A nonlinear optimal control problem for assimilation of surface data in a Navier-Stokes type equations related to oceanography, Numer. Funct. Anal. Optim. 20 (1999), 1-26.

[6] Belmiloudi, A., Regularity results and optimal control problems for the perturbation of Boussinesq equations of the ocean, Numer. Funct. Anal. Optim. 21 (2000), 623-651.

[7] Belmiloudi, A., Existence and characterization of an optimal control for the problem of long waves in a shallow-water model, SIAM J. Control Optim. 39(5) (2001), 15581584 .

[8] Belmiloudi, A., Robin-type boundary control problems for the nonlinear Boussinesq type equations, J. Math. Anal. Appl. 273 (2002), 428-456.

[9] Belmiloudi, A., Brossier, F., Regularity results for a Navier-Stokes type problem related to oceanography, Acta Appl. Math. 48 (1997), 299-316.

[10] Belmiloudi, A., Brossier, F., A control method for assimilation of surface data in a linearized Navier-Stokes type problem related to oceanography, SIAM J. Control Optim. 35 (1997) 2183-2197.

[11] Belmiloudi, A., Brossier, F., Monier, L., Mathematical and numerical modellization of large-scale oceanic waves, Math. Methods Appl. Sci. 22 (1999), 967-999.

[12] Brossier, F., Monier, L., Delecluse, P., Simulation of long equatorial waves in the Pacific ocean in relation with sea level oscillations and zonal mean currents, Oceanologica Acta 17 (1994), 461-477.

[13] Brezis, H., Analyse Fonctionnelle, Théorie et Application, Masson, Paris, 1983.

[14] Dauge, M., Elliptic Boundary Value Problems on Corners Domain, Lecture Notes in Math. 1341, Springer Verlag, New York, 1988.

[15] Dauge, M., Problmes mixtes pour le la placien dans des domaines polydraux courbes, C. R. Acad. Sci. Paris Sér. I Math. 309(8) (1989), 553-558.

[16] Grisvard, P., Elliptic Problems in Nonsmooth Domains, Monographs and Studies in Mathematics 24, Pitman, Boston, 1985.

[17] Lions, J. L., Contrôle Optimal de Systèmes Gouvernés par des Équations aux Dérivées Partielles, Dunod, Paris, 1968.

[18] Lions, J. L., Équations Différentielles Opérationnelles et Problèmes aux Limites, Springer Verlag, Berlin, 1961.

[19] Lions, J. L., Temam, R., Wang, S., New formulation of the primitive equations of atmosphere and application, Nonlinearity 5 (1992), 237-288.

[20] Lions, J. L., Temam, R., Wang, S., On the equation of the large-scale ocean, Nonlinearity 5 (1992), 1007-1053.

[21] Marchuk, G. I., Mathematical Models in Envirenmental Problems, Studies in Mathematics and its Applications 16, North-Holland Publishing, Amsterdam, 1986.

[22] Marchuk, G. I., Adjoint Equation and Analysis of Complex Systems, Mathematics and Its Applications 295, Kluwer Academic Publishers Group, Dordrecht, 1995.

[23] Philander, S. G. H., Pacanowski, R. C., The generation of equatorial currents, J. Geophys. Res. 85 (1980), 1123-1136.

[24] Philander, S. G. H., Pacanowski, R. C., Parameterization of vertical mixing in numerical models of tropical oceans, J. Phys. Oceanogr. 11 (1981), 1443-1451.

[25] Temam, T., Navier-Stokes Equations, North-Holland Publishing, Amsterdam, 1977.

[26] Ziane, M. Regularity results for the stationary primitive equations of the atmosphere and the ocean, Nonlinear Anal. 28(2) (1997), 289-313. 
AzIz Belmiloudi

IRMAR-UnIVERsité RenNes1

CENTRE DE MATHS INSA DE RENNES

20 AVenue Des Buttes de Cö̈smes

CS 14315, 35043 Rennes CÉdex

FRANCE

E-MAil: AzIz.Belmiloudi@InsA-REnNes.Fr 